CRYSTALLOGRAPHIC COMMUNICATIONS

ISSN 2056-9890

Received 7 August 2019

Accepted 18 October 2019

Edited by E. V. Boldyreva, Russian Academy of Sciences, Russia

Keywords: crystal structure; suberone; polymorphism.

CCDC references: 1960122; 1960121; $1960120 ; 1960119 ; 1960118$

Supporting information: this article has supporting information at journals.iucr.org/e

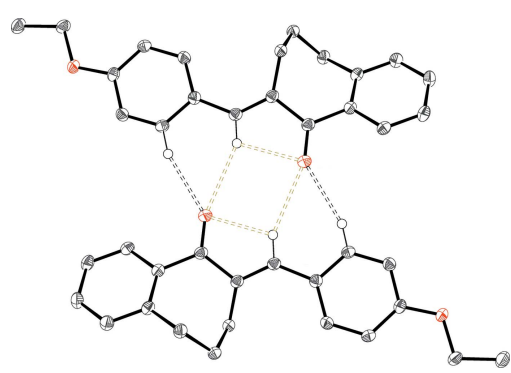

OPEN $\odot$ ACCESS

\section{Different packing motifs mediated by weak inter- actions and polymorphism in the crystal structures of five 2-(benzylidene)benzosuberone derivatives}

Lewis S. Seaman, ${ }^{a}$ Cristiane F. da Costa, ${ }^{b}$ Marcus V. N. de Souza, ${ }^{b}$ Solange M. S. V. Wardell, ${ }^{c}$ James L. Wardell ${ }^{\mathrm{a}}$ and William T. A. Harrison ${ }^{\mathrm{a} *}$

\footnotetext{
${ }^{\mathbf{a}}$ Department of Chemistry, University of Aberdeen, Meston Walk, Aberdeen AB24 3UE, Scotland, ${ }^{\mathbf{b}}$ Fundação Oswaldo Cruz, Instituto de Tecnologia em Fármacos Manguinhos, 21041-250 Rio de Janeiro, RJ, Brazil, and ${ }^{\mathrm{c} C H E M S O L, ~}$

1 Harcourt Road, Aberdeen AB15 5NY, Scotland. *Correspondence e-mail: w.harrison@abdn.ac.uk
}

The syntheses and crystal structures of five 2-benzylidene-1-benzosuberone [1-benzosuberone is 6,7,8,9-tetrahydro-5H-benzo[7]annulen-5-one] derivatives, viz. 2-(4-methoxybenzylidene)-1-benzosuberone, $\mathrm{C}_{19} \mathrm{H}_{18} \mathrm{O}_{2}$, (I), 2-(4-ethoxybenzylidene)-1-benzosuberone, $\mathrm{C}_{20} \mathrm{H}_{20} \mathrm{O}_{2}$, (II), 2-(4-benzylbenzylidene)-1benzosuberone, $\mathrm{C}_{25} \mathrm{H}_{22} \mathrm{O}_{2}$, (III), 2-(4-chlorobenzylidene)-1-benzosuberone, $\mathrm{C}_{18} \mathrm{H}_{15} \mathrm{ClO}$, (IV) and 2-(4-cyanobenzylidene)-1-benzosuberone, $\mathrm{C}_{19} \mathrm{H}_{15} \mathrm{NO}$, (V), are described. The conformations of the benzosuberone fused six- plus seven-membered ring fragments are very similar in each case, but the dihedral angles between the fused benzene ring and the pendant benzene ring differ somewhat, with values of 23.79 (3) for (I), 24.60 (4) for (II), 33.72 (4) for (III), 29.93 (8) for (IV) and $21.81(7)^{\circ}$ for (V). Key features of the packing include pairwise $\mathrm{C}-\mathrm{H} \cdots \mathrm{O}$ hydrogen bonds for (II) and (IV), and pairwise $\mathrm{C}-\mathrm{H} \cdots \mathrm{N}$ hydrogen bonds for (V), which generate inversion dimers in each case. The packing for (I) and (III) feature $\mathrm{C}-\mathrm{H}$. . O hydrogen bonds, which lead to [010] and [100] chains, respectively. Weak $\mathrm{C}-\mathrm{H} \cdots \pi$ interactions consolidate the structures and weak aromatic $\pi-\pi$ stacking is seen in (II) [centroid-centroid separation $=3.8414$ (7) $\AA$ ] and (III) [3.9475 (7) $\AA$ ]. A polymorph of (I) crystallized from a different solvent has been reported previously [Dimmock et al. (1999) J. Med. Chem. 42, 1358-1366] in the same space group but with a packing motif based on inversion dimers resembling that seen in (IV) in the present study. The Hirshfeld surfaces and fingerprint plots for (I) and its polymorph are compared and structural features of the 2-benzylidene-1benzosuberone family of phases are surveyed.

\section{Chemical context}

The structurally related 2-benzylidenebenzocycloalkanone compounds, viz. (E)-2-benzylidene-2,3-dihydro-1H-inden-1one $(n=1),(E)$-2-benzylidene-1-tetralone $(n=2)$ and $(E)$-2benzylidene-1-benzosuberone $(n=3)$, which differ with respect to the number of methylene groups, $n$, in the alkanone ring fused to the benzene ring (see Scheme 1) have attracted attention in a number of areas. Their biological activities include antitumour (e.g. Gautam et al., 2016: Dimmock et al., 1999, 2002), antimycotic (Al-Nakib et al., 1997) and antifungal (Gupta \& Jain, 2015) properties. Their physical properties include nonlinear optical (Watson et al., 1993) and UV hypsochromic shifts and fluorescence effects (Fodor et al., 2011). It may be noted that these compounds can be considered as fused-ring analogues of chalcones (i.e. the ' $n=0$ ' family), which might allow for 'tuneable' conformational control of the molecule by changing the number of methylene groups in the cycloalkanone ring (Dimmock et al., 1999). 
In continuation of our earlier reports of the crystal structures and Hirshfeld surface analyses of a number of $(E)$-2benzylidene-2,3-dihydro- $1 H$-inden-1-one derivatives (Baddeley et al., 2017a) and (E)-2-benzylidene-1-tetralone (Baddeley et $a l ., 2017 b)$, we now describe the syntheses and crystal structures of 2-(4-methoxybenzylidene)-1-benzosuberone, (I), 2-(4ethoxybenzylidene)-1-benzosuberone, (II), 2-(4-benzylbenzylidene)-1-benzosuberone, (III), 2-(4-chlorobenzylidene)-1-benzosuberone, (IV), and 2-(4-cyanobenzylidene)-1-benzosuberone, (V) (see Scheme 2).<smiles>[X]c1cccc(C=C(C)C(=O)c2ccccc2C)c1</smiles>

Scheme 1<smiles>[X]c1ccc(/C=C2\CCCc3ccccc3C2=O)cc1</smiles>

(I) $\mathrm{X}=\mathrm{OMe}$

(II) $\mathrm{X}=\mathrm{OEt}$

(III) $\mathrm{X}=\mathrm{OBz}$

(IV) $\mathrm{X}=\mathrm{Cl}$

Scheme 2

(V) $\mathrm{X}=\mathrm{CN}$

\section{Structural commentary}

The molecular structures of (I)-(V) are shown in Figs. 1-5, respectively. Each molecule is the expected product arising

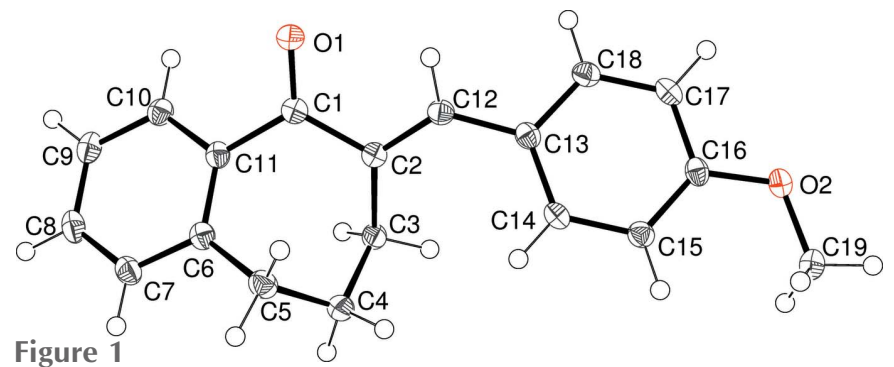

The molecular structure of (I), showing $50 \%$ probability displacement ellipsoids.

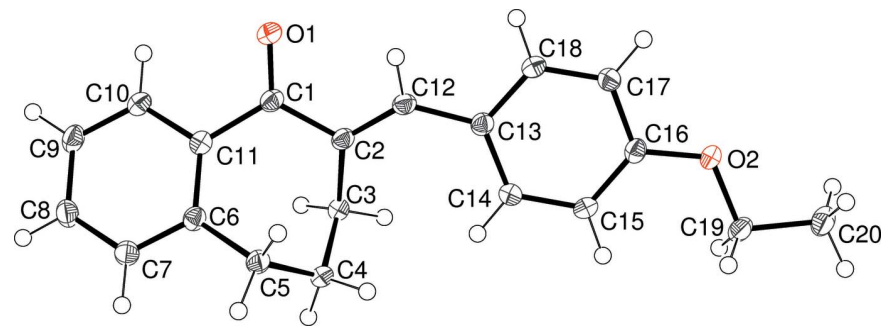

Figure 2

The molecular structure of (II), showing $50 \%$ probability displacement ellipsoids.

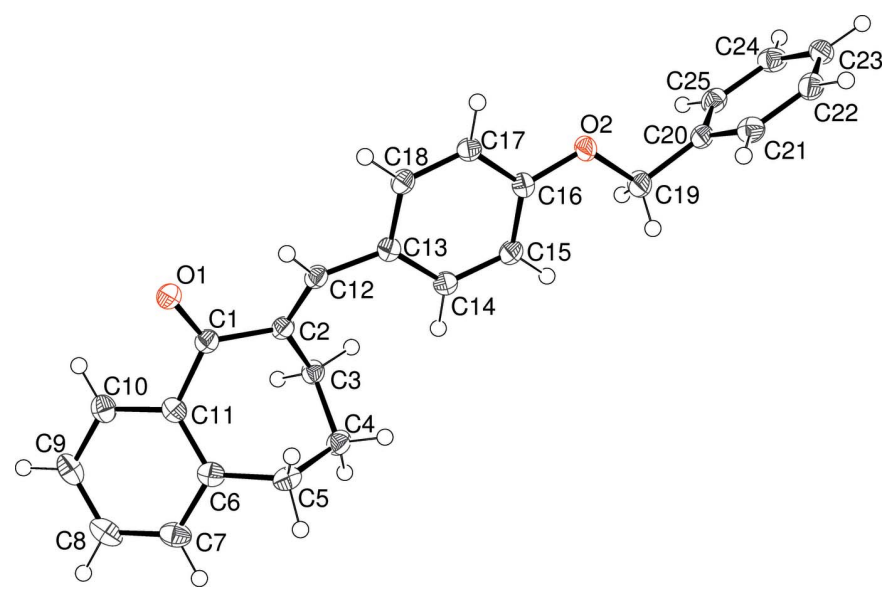

Figure 3

The molecular structure of (III), showing $50 \%$ probability displacement ellipsoids.

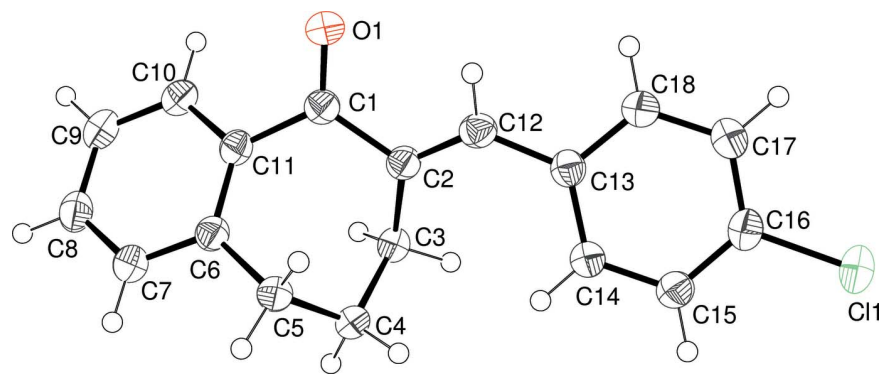

Figure 4

The molecular structure of (IV), showing $50 \%$ probability displacement ellipsoids.

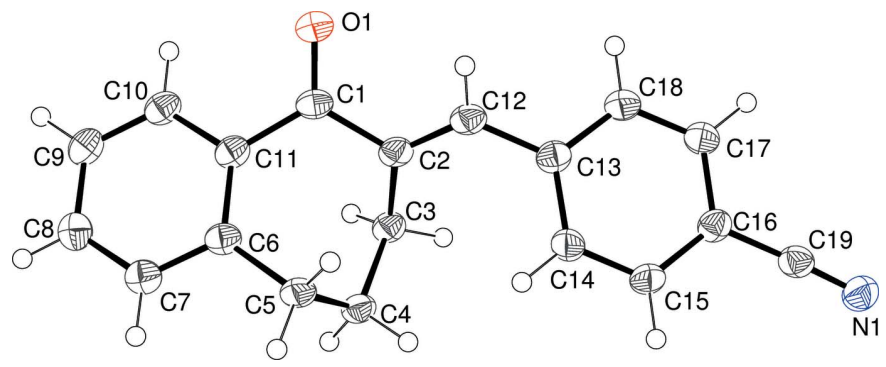

Figure 5

The molecular structure of $(\mathrm{V})$, showing $50 \%$ probability displacement ellipsoids.

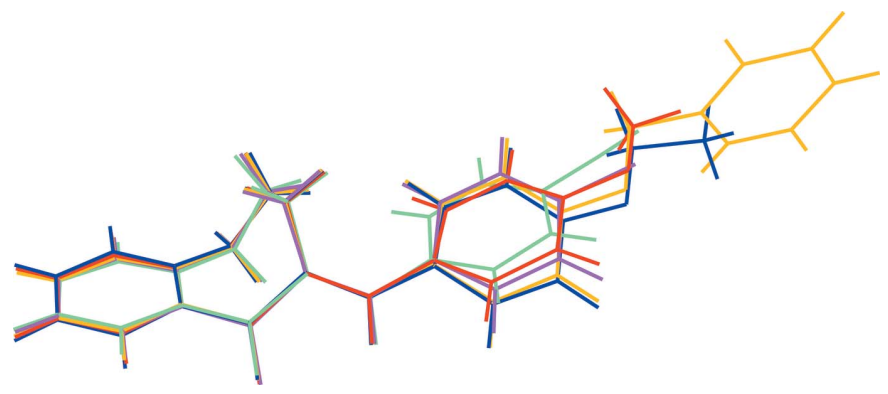

Figure 6

Overlay plot for (I)-(V), with (I) red, (II) blue, (III) orange, (IV) purple and $(\mathrm{V})$ green. 


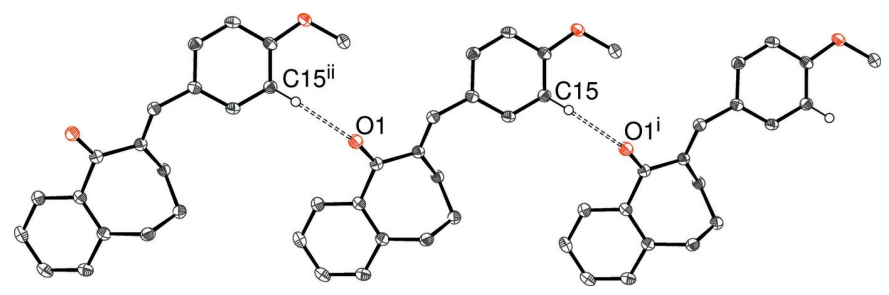

Figure 7

Fragment of the crystal structure of (I), showing part of a [010] chain linked by $\mathrm{C} 15-\mathrm{H} 15 \cdots \mathrm{O} 1$ hydrogen bonds. [Symmetry codes: (i) $x, y-1$, $z$; (ii) $x, y+1, z$.]

from the base-catalysed condensation reaction between 1-benzosuberone and the appropriate 4-substituted benzaldehyde derivative (see Experimental section). The conformations of the benzosuberone fragments in (I)-(V) are almost identical, as shown by the overlay plot generated with $Q M O L$ (Gans \& Shalloway, 2001) shown in Fig. 6. The sevenmembered ring, which is conformationally constrained by being fused to the $\mathrm{C} 6-\mathrm{C} 11$ benzene ring and by the presence of the $s p^{2}$-hybridized atoms $\mathrm{C} 1$ and $\mathrm{C} 2$, at least approximates to a boat conformation; in the case of (I), atoms C3/C4/C6/C11 are roughly coplanar (r.m.s. deviation $=0.095 \AA$ ), with C5 as the prow [deviation $=0.6139(15) \AA$ ] and $\mathrm{C} 1$ and $\mathrm{C} 2$ as the stern [deviations $=1.0114(16)$ and $1.0154(16) \AA$, respectively]. This conformation results in a substantial degree of twist about the $\mathrm{C} 11-\mathrm{C} 1$ bond $[\mathrm{C} 10-\mathrm{C} 11-\mathrm{C} 1=\mathrm{O} 1=$ $36.06(14)^{\circ}$ ] and $\mathrm{O} 1$ deviates from the C6-C11 benzene-ring plane by 0.7212 (17) $\AA$. The corresponding data for the sevenmembered rings in (II)- $(\mathrm{V})$ are very similar to those for (I) and are not stated here.

The dihedral angles between the C6-C11 fused benzene ring and the $\mathrm{C} 13-\mathrm{C} 18$ pendant benzene ring are clustered in a $\sim 12^{\circ}$ range, with values of 23.79 (3) for (I), 24.60 (4) for (II), 33.72 (4) for (III), 29.93 (8) for (IV) and $21.81(7)^{\circ}$ for (V). A comparison of the $\mathrm{C} 1-\mathrm{C} 2-\mathrm{C} 12-\mathrm{C} 13$ and $\mathrm{C} 2-\mathrm{C} 12-\mathrm{C} 13-$ C14 torsion angles for (I) $\left[-179.67(10)\right.$ and $-33.81(17)^{\circ}$,

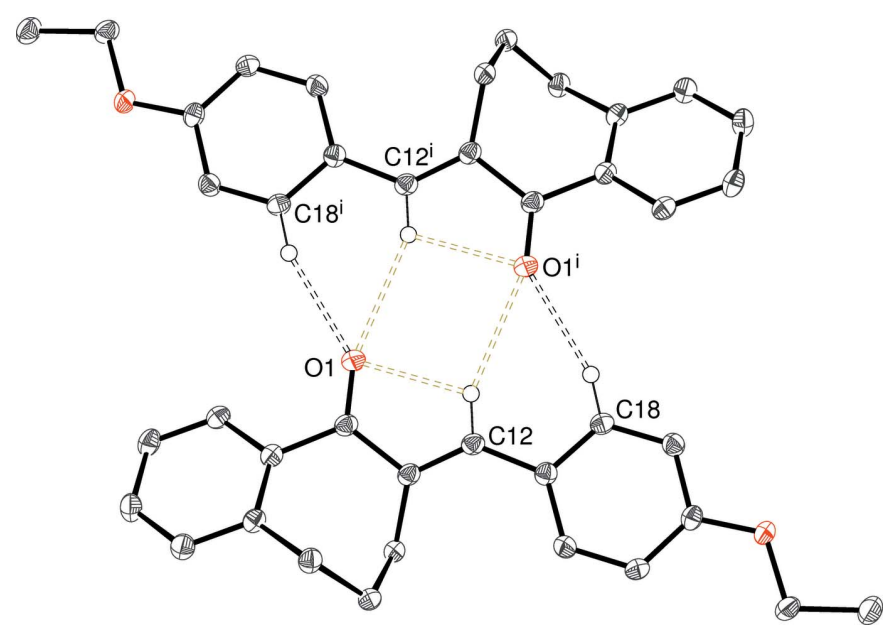

Figure 8

Fragment of the crystal structure of (II), showing inversion dimers linked by pairs of $\mathrm{C} 18-\mathrm{H} 18 \cdots \mathrm{O} 1$ hydrogen bonds. [Symmetry code: (i) $-x+1$, $-y+1,-z+1$.]
Table 1

Hydrogen-bond geometry $\left(\AA,^{\circ}\right)$ for (I).

$C g 1$ and $C g 2$ are the centroids of the $\mathrm{C} 6-\mathrm{C} 11$ and $\mathrm{C} 13-\mathrm{C} 18$ rings, respectively.

\begin{tabular}{lllll}
\hline$D-\mathrm{H} \cdots A$ & $D-\mathrm{H}$ & $\mathrm{H} \cdots A$ & $D \cdots A$ & $D-\mathrm{H} \cdots A$ \\
\hline $\mathrm{C} 15-\mathrm{H} 15 \cdots \mathrm{O} 1^{\mathrm{i}}$ & 0.95 & 2.35 & $3.2971(14)$ & 176 \\
$\mathrm{C} 19-\mathrm{H} 19 A \cdots C g 1^{\mathrm{ii}}$ & 0.98 & 2.76 & $3.6165(13)$ & 146 \\
$\mathrm{C} 19-\mathrm{H} 19 C \cdots C g 2^{\mathrm{iii}}$ & 0.98 & 2.74 & $3.6029(13)$ & 147 \\
\hline
\end{tabular}

Symmetry codes: (i) $x, y-1, z$; (ii) $x,-y+\frac{1}{2}, z+\frac{1}{2}$; (iii) $-x+1,-y,-z+1$.

Table 2

Hydrogen-bond geometry $\left(\AA{ }^{\circ}\right)$ for (II).

$C g 1$ and $C g 2$ are the centroids of the $\mathrm{C} 6-\mathrm{C} 11$ and $\mathrm{C} 13-\mathrm{C} 18$ rings, respectively.

\begin{tabular}{lllll}
\hline$D-\mathrm{H} \cdots A$ & $D-\mathrm{H}$ & $\mathrm{H} \cdots A$ & $D \cdots A$ & $D-\mathrm{H} \cdots A$ \\
\hline $\mathrm{C} 18-\mathrm{H} 18 \cdots \mathrm{O} 1^{\mathrm{i}}$ & 0.95 & 2.36 & $3.2653(14)$ & 159 \\
$\mathrm{C} 4-\mathrm{H} 4 B \cdots C g 1^{\mathrm{ii}}$ & 0.98 & 2.72 & $3.6429(13)$ & 155 \\
$\mathrm{C} 19-\mathrm{H} 19 A \cdots C g 2^{\mathrm{ii}}$ & 0.98 & 2.71 & $3.5969(13)$ & 149 \\
\hline
\end{tabular}

Symmetry codes: (i) $-x+1,-y+1,-z+1$; (ii) $x,-y+\frac{1}{2}, z+\frac{1}{2}$.

Table 3

Hydrogen-bond geometry $\left(\stackrel{\AA}{\mathrm{A}}{ }^{\circ}\right)$ for (III).

$\mathrm{Cg} 3$ is the centroid of the $\mathrm{C} 20-\mathrm{C} 25$ ring.

\begin{tabular}{lllll}
\hline$D-\mathrm{H} \cdots A$ & $D-\mathrm{H}$ & $\mathrm{H} \cdots A$ & $D \cdots A$ & $D-\mathrm{H} \cdots A$ \\
\hline $\mathrm{C} 15-\mathrm{H} 15 \cdots \mathrm{O} 1^{\mathrm{i}}$ & 0.95 & 2.40 & $3.3477(13)$ & 176 \\
$\mathrm{C} 18-\mathrm{H} 18 \cdots C g 3^{\mathrm{ii}}$ & 0.95 & 2.64 & $3.5147(13)$ & 153
\end{tabular}

Symmetry codes: (i) $x+1, y, z$; (ii) $x-1, y, z$.

Table 4

Hydrogen-bond geometry $\left(\AA{ }^{\circ}\right)$ for (IV).

$C g 1$ is the centroid of the C6-C11 ring.

\begin{tabular}{lllll}
\hline$D-\mathrm{H} \cdots A$ & $D-\mathrm{H}$ & $\mathrm{H} \cdots A$ & $D \cdots A$ & $D-\mathrm{H} \cdots A$ \\
\hline $\mathrm{C} 10-\mathrm{H} 10 \cdots \mathrm{O} 1^{\mathrm{i}}$ & 0.95 & 2.50 & $3.319(2)$ & 145 \\
$\mathrm{C} 3-\mathrm{H} 3 A \cdots C g 1^{\mathrm{ii}}$ & 0.99 & 2.83 & $3.572(2)$ & 132 \\
\hline
\end{tabular}

Symmetry codes: (i) $-x,-y+1,-z+1$; (ii) $x-\frac{1}{2},-y+\frac{3}{2}, z+\frac{1}{2}$.

Table 5

Hydrogen-bond geometry $\left(\AA,^{\circ}\right)$ for $(\mathrm{V})$.

$\mathrm{Cg} 1$ is the centroid of the C6-C11 ring.

\begin{tabular}{lllll}
\hline$D-\mathrm{H} \cdots A$ & $D-\mathrm{H}$ & $\mathrm{H} \cdots A$ & $D \cdots A$ & $D-\mathrm{H} \cdots A$ \\
\hline $\mathrm{C} 17-\mathrm{H} 17 \cdots \mathrm{N} 1^{\mathrm{i}}$ & 0.95 & 2.54 & $3.438(2)$ & 157 \\
$\mathrm{C} 3-\mathrm{H} 3 A \cdots C g 1^{\mathrm{ii}}$ & 0.99 & 2.84 & $3.6730(16)$ & 142 \\
$\mathrm{C} 8-\mathrm{H} 8 \cdots C g 1^{\mathrm{iii}}$ & 0.95 & 2.88 & $3.7868(17)$ & 161 \\
\hline
\end{tabular}

Symmetry codes: (i) $-x,-y-1,-z+1$; (ii) $x, y-1, z$; (iii) $-x+1, y+\frac{1}{2},-z+\frac{1}{2}$.

respectively] indicates that the twisting largely occurs about the $\mathrm{C} 12-\mathrm{C} 13$ bond, and the same conclusion can be drawn for (II)-(V).

For (I), the C19 atom of the methoxy group is close to coplanar with its attached benzene ring [deviation = 0.1079 (19) §] and for (II) the ethoxy group has an extended conformation $\left[\mathrm{C} 16-\mathrm{O} 2-\mathrm{C} 19-\mathrm{C} 20=178.58(10)^{\circ}\right]$. For (III), an additional dihedral angle between the C13-C18 benzene ring and the terminal $\mathrm{C} 20-\mathrm{C} 25$ benzene ring of $78.78(3)^{\circ}$ is 
Table 6

Fingerprint contact percentages for (I) and VENQUA.

\begin{tabular}{lll}
\hline Contact type & $(\mathrm{I})$ & VENQUA \\
\hline $\mathrm{H} \cdots \mathrm{H}$ & 54.8 & 55.3 \\
$\mathrm{C} \cdots \mathrm{H} / \mathrm{H} \cdots \mathrm{C}$ & 28.1 & 29.2 \\
$\mathrm{O}-\mathrm{H} / \mathrm{H} \cdots \mathrm{O}$ & 15.3 & 14.5 \\
$\mathrm{C} \cdots \mathrm{C}$ & 1.1 & 0.0 \\
$\mathrm{C} \cdots \mathrm{O} / \mathrm{O} \cdots \mathrm{C}$ & 0.8 & 0.8 \\
$\mathrm{O} \cdots \mathrm{O}$ & 0.0 & 0.2 \\
\hline
\end{tabular}

observed. Otherwise, the geometrical data for (I)-(V) are unexceptional and similar to those for related compounds (Dimmock et al., 1999, 2002).

It may be noted that a polymorph of (I) [Cambridge Structural Database (CSD; Groom et al., 2016) refcode VENQUA; Dimmock et al., 1999] has been reported in the same space group, i.e. $P 2_{1} / c$; VENQUA was recrystallized from methanol solution rather than ethanol for (I). The bond lengths and angles in (I) and VENQUA are very similar, although there is a $\sim 10^{\circ}$ difference in the dihedral angle between the benzene rings [value for VENQUA $=35.88(11)^{\circ}$, calculated with PLATON (Spek, 2009)]; for an overlay plot of (I) and VENQUA, see the supporting information.

\section{Supramolecular features}

There are obviously no classical hydrogen bonds in these structures and, in each case, just one $\mathrm{C}-\mathrm{H}$ group can be identified as the donor for a weak hydrogen bond with atom $\mathrm{O} 1$ as the acceptor in (I)-(IV) and atom N1 in (V); geometrical data for these interactions are listed in Tables 1-5 and illustrated in Figs. 7-11. All the structures also feature weak $\mathrm{C}-\mathrm{H} \cdots \pi$ interactions with either the fused or pendant benzene rings as acceptors, but (II) and (III) are the only structures to display weak aromatic $\pi-\pi$ stacking, in both cases between inversion-related C13-C18 rings. For (II), the

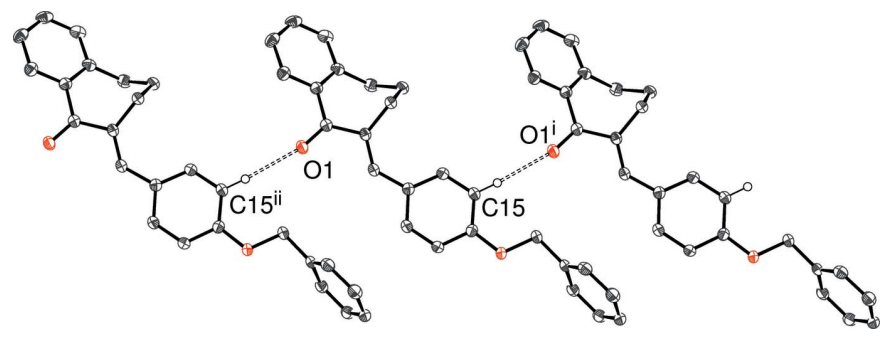

Figure 9

Fragment of the crystal structure of (III), showing part of a [100] chain linked by $\mathrm{C} 15-\mathrm{H} 15 \cdots \mathrm{O} 1$ hydrogen bonds. [Symmetry codes: (i) $x+1, y$, $z$; (ii) $x-1, y, z$.]

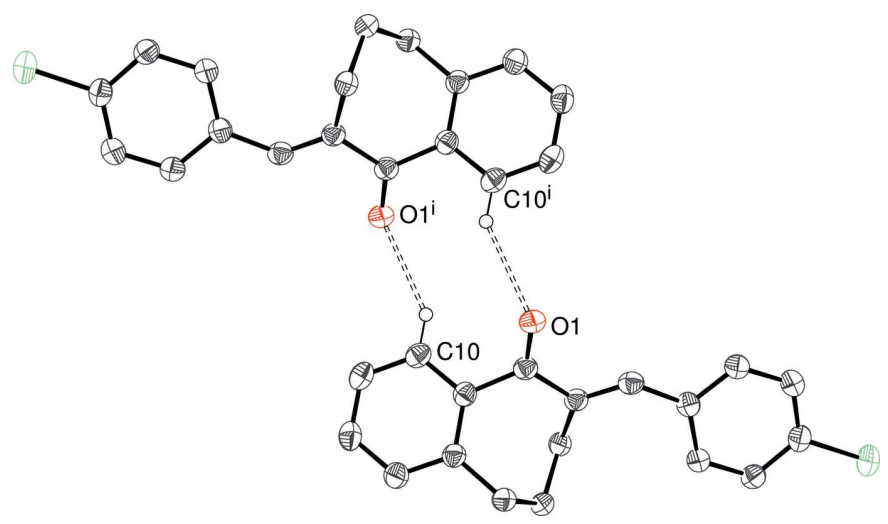

Figure 10

Fragment of the crystal structure of (IV), showing inversion dimers linked by pairs of $\mathrm{C} 10-\mathrm{H} 10 \cdots \mathrm{O} 1$ hydrogen bonds. [Symmetry code: (i) $-x$, $-y+1,-z+1$.]

centroid-centroid separation is 3.8414 (7) $\AA$ and the slippage is $1.72 \AA$; equivalent data for (III) are 3.9475 (7) and $1.89 \AA$, respectively.

The packing motifs for the extended structures of (I) and (III) are infinite $\mathrm{C}-\mathrm{H} \cdots \mathrm{O}$ hydrogen-bonded chains, which propagate in the [010] and [100] directions, respectively. In each case, adjacent molecules are related only by unit-cell

Table 7

Summary of the $\mathrm{C}-\mathrm{H} \cdots \mathrm{O}$ and $\mathrm{C}-\mathrm{H} \cdots \mathrm{N}$ hydrogen bonds and packing motifs for 2-(benzylidene)benzosuberone derivatives.

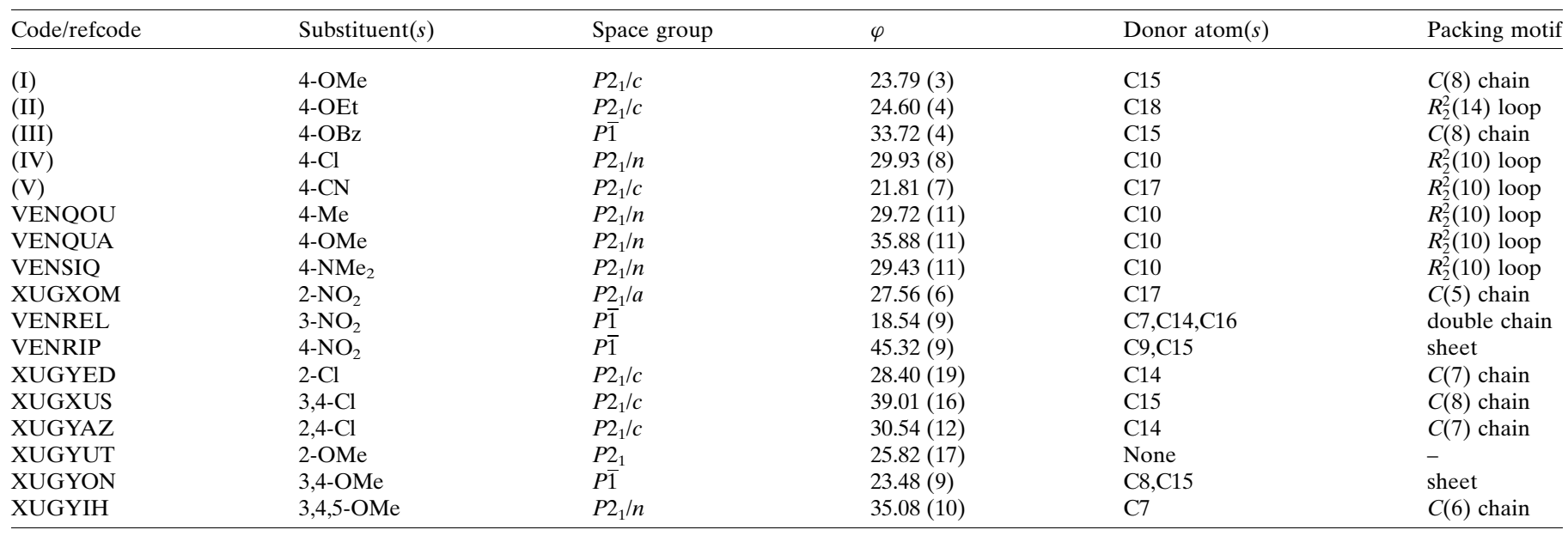

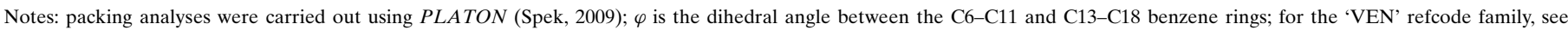
Dimmock et al. (1999); for the 'XUG' family, see Dimmock et al. (2002); the donor atom labels correspond to our atom numbering scheme. 


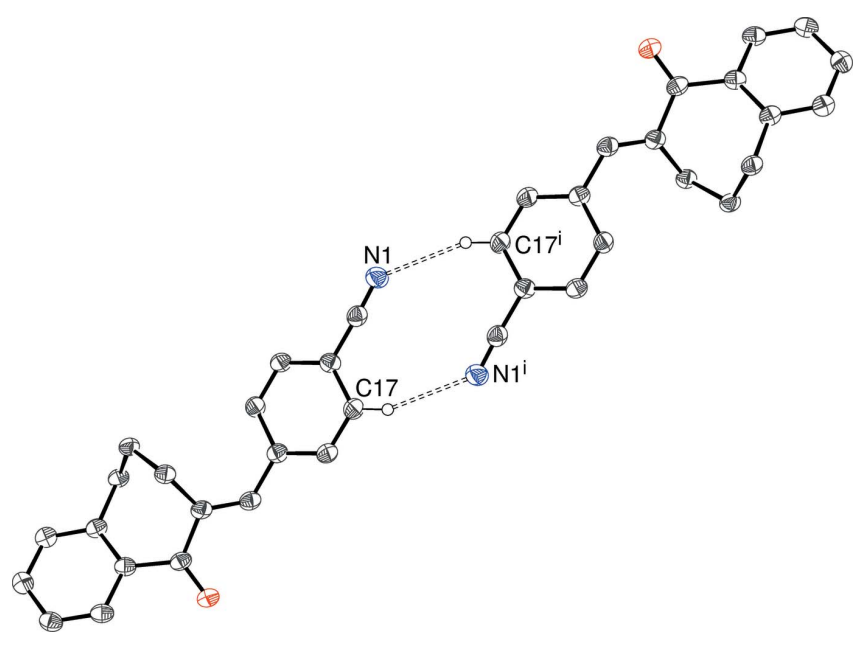

Figure 11

Fragment of the crystal structure of $(\mathrm{V})$, showing inversion dimers linked by pairs of $\mathrm{C} 17-\mathrm{H} 17 \cdots \mathrm{N} 1$ hydrogen bonds. [Symmetry code: $-x,-y+1$, $-z+1$.]

translational symmetry and a $C(8)$ graph-set motif results for both structures with the methyne group $(\mathrm{C} 15-\mathrm{H} 15$, ortho to the 4-substituent) involved as the donor.

The packing motifs for (II) and (IV) feature inversion dimers. In (II), C18-H18 (meta to the 4-substituent) is the donor group and $R_{2}^{2}(14)$ loops arise. In this motif, C12-H12 is 'sandwiched' between the donor and acceptor and the H12. . O 1 separation of $2.60 \AA$ (see Fig. 8) is borderline to be regarded as a directional bond. The donor group in (IV) is $\mathrm{C} 10-\mathrm{H} 10$ in the fused benzene ring, which generates an $R_{2}^{2}(10)$ loop. The only possible interaction involving the $\mathrm{Cl}$ atom is a long contact from $\mathrm{C} 8-\mathrm{H} 8$, with $\mathrm{H} \cdots \mathrm{Cl}=2.93 \AA$. The presence of the cyano group in (V) allows for the formation of pairwise $\mathrm{C}-\mathrm{H} \cdots \mathrm{N}$ hydrogen bonds and an $R_{2}^{2}(10)$ graph-set motif arises; the shortest $\mathrm{H} \cdots \mathrm{O}$ contact in (V) is $2.72 \AA$.

Rather than the $C(8)$ chains arising from $\mathrm{C} 15-\mathrm{H} 15 \cdots \mathrm{O} 1$ hydrogen bonds seen in (I), the packing for VENQUA (see above) features inversion dimers built from pairwise $\mathrm{C} 10-$ $\mathrm{H} 10$. . O 1 interactions, which are very similar to those seen in 4-chloro derivative (IV) in the present study. It may be noted that the density of VENQUA $\left(\rho=1.368 \mathrm{Mg} \mathrm{m}^{-3}\right)$ is significantly greater than that of (I) $\left(\rho=1.284 \mathrm{Mg} \mathrm{m}^{-3}\right)$, suggesting that the former might be the more stable polymorph if the 'rational packing rule' (Kitaigorodskii, 1961) is applicable in this case.

In order to gain further insight into these different packing motifs, the Hirshfeld surfaces and fingerprint plots for (I) and VENQUA were calculated using CrystalExplorer (Turner et al., 2017), following the approach recently described by Tan et al. (2019). The Hirshfeld surface for (I) (see supporting information) shows the expected large red spots (close contacts) in the vicinity of $\mathrm{H} 15$ and $\mathrm{O} 1$ corresponding to the $\mathrm{C} 15-\mathrm{H} 15 \cdots \mathrm{O} 1$ interaction noted above, but there is little if any evidence of close contacts in the vicinity of $\mathrm{H} 19 A$ and $\mathrm{H} 19 C$ corresponding to the $\mathrm{C}-\mathrm{H} \cdots \pi$ contacts listed in Table 1 . The surface for VENQUA (see supporting informa- tion) shows red spots in the vicinity of $\mathrm{H} 10$ and $\mathrm{O} 1$ corresponding to the $\mathrm{C} 10-\mathrm{H} 10 \cdots \mathrm{O} 1$ hydrogen bond and $\mathrm{H} 2 A$ (our numbering scheme) corresponding to a $\mathrm{C} 3-\mathrm{H} 2 A \cdots \pi$ interaction $(\mathrm{H} \cdots \pi=2.69 \AA)$ to the centroid of the $\mathrm{C} 6-\mathrm{C} 11$ benzene ring, but there are also probably spurious features close to $\mathrm{H} 8$ and $\mathrm{H} 17$ corresponding to a short $\mathrm{H}$. . H contact of $2.07 \AA$ between these atoms, which possibly arose because the $\mathrm{H}$ atoms of the $\mathrm{C} 19$ methyl group in VENQUA were geometrically placed and not treated using a rotating-group model. Notwithstanding this, the fingerprint plots for (I) and VENQUA (see supporting information) decomposed into the different percentage contact types (Table 6) are almost identical; $\mathrm{H} \cdots \mathrm{H}$ (van der Waals) contacts dominate both structures, followed by $\mathrm{C} \cdots \mathrm{H} / \mathrm{H} \cdots \mathrm{C}$ and then $\mathrm{O} \cdots \mathrm{H} / \mathrm{H} \cdots \mathrm{O}$. The percentage contributions of the other contact types are negligible.

\section{Database survey}

A survey of the Cambridge Structural Database (CSD; Groom et al., 2016) revealed 167 structures incorporating a 1-benzosuberone fragment but only 20 hits when an exocyclic $\mathrm{C}=\mathrm{C}$ double bond at the 2-position was added to the search structure. The key papers reporting the structures of closely related, differently substituted, 2-benzylidene-1-benzosuberones are Dimmock et al. (1999, 2002). The hydrogen-bond data for (I)-(V) and the 12 structures reported in the two papers by Dimmock et al. are summarized in Table 7. The most frequently observed motif is the centrosymmetric $R_{2}^{2}(10)$ loop involving $\mathrm{C} 10-\mathrm{H} 10$ as the donor group, but there are many others involving different $\mathrm{C}-\mathrm{H}$ groups as donor and we see no obvious connection to the nature and position of the substituent(s) on the remote benzene ring. There are no structures in which the fused and pendant benzene rings tend towards being perpendicular (dihedral angle $>60^{\circ}$ ).

The fact that (I) and VENQUA have similar conformations but distinct packing motifs mediated by different $\mathrm{C}-\mathrm{H}$...O interactions to the same acceptor $\mathrm{O}$ atom may be compared with the fascinating recent survey of weak-interaction polymorphs by Lo Presti (2018). He concluded that weak hydrogen bonds and solvent effects may play an important kinetic role in promoting polymorph formation (after all, something has to favour a situation where the lowest-energy packing motif is not adopted) but they do not play a dominant energetic role in polymorph formation and that the overall energy balance between dispersive (attractive) and repulsive interactions is the most important consideration.

\section{Synthesis and crystallization}

Compounds (I)-(V) were obtained from the reaction of 1-benzosuberone $(1 \mathrm{mmol})$ with the appropriate 4 -substituted benzaldehyde $(1 \mathrm{mmol})$ in ethanol $(5 \mathrm{ml})$ treated with an ethanolic solution of sodium hydroxide $(30 \mathrm{mg}$ in $5 \mathrm{ml}$ ethanol). After stirring for 3-4 h at room temperature, each reaction mixture was cooled to $0{ }^{\circ} \mathrm{C}$ and the precipitated solid was recovered by filtration and rinsing with ice-cold ethanol. 
Table 8

Experimental details.

(I) (II)
Crystal data

Chemical formula

$M_{\mathrm{r}}$

Crystal system, space group

Temperature (K)

$a, b, c(\AA)$

$\alpha, \beta, \gamma\left({ }^{\circ}\right)$

$Z$

Radiation type

$\mu\left(\mathrm{mm}^{-1}\right)$

Crystal size (mm)

Data collection

Diffractometer

Absorption correction

$T_{\min }, T_{\max }$

No. of measured, independent and observed $[I>2 \sigma(I)]$ reflections

$R_{\text {int }}$

$(\sin \theta / \lambda)_{\max }\left(\AA^{-1}\right)$
$\mathrm{C}_{19} \mathrm{H}_{18} \mathrm{O}_{2}$

278.33

Monoclinic, $P 2_{1} / c$

100

10.9171 (3), 9.1262 (2), 15.2539 (3)

90, $108.618(3), 90$

$1440.24(6)$

4

Mo $K \alpha$

0.08

$0.20 \times 0.15 \times 0.05$

XtaLAB AFC12 (RCD3): Kappa single CCD

Multi-scan (CrysAlis PRO; Rigaku, 2017)

$0.877,1.000$

16988, 3296, 2843

0.033

0.649

$0.037,0.093,1.03$

3296

191

$\mathrm{H}$-atom parameters constrained $0.25,-0.18$

$\mathrm{C}_{20} \mathrm{H}_{20} \mathrm{O}_{2}$
292.36
Monoclinic, $P 2_{1} / c$
100
$12.6208(2), 14.99690(17)$,
$\quad 8.39151(12)$
$90,108.6814(17), 90$
$1504.60(4)$
4
$\mathrm{Cu} \mathrm{K \alpha}$
0.64
$0.20 \times 0.11 \times 0.03$

(III)

Refinement

$R\left[F^{2}>2 \sigma\left(F^{2}\right)\right], w R\left(F^{2}\right), S$

No. of reflections

No. of parameters

$\Delta \rho_{\max }, \Delta \rho_{\min }\left(\mathrm{e} \AA^{-3}\right)$

XtaLAB AFC11 (RCD3): quarterchi single CCD

Gaussian (CrysAlis PRO; Rigaku, 2017)

$0.772,1.000$

9197, 2704, 2486

0.024

0.602

100

2

0.63

$2704 \quad 3336$

$201 \quad 245$ $0.25,-0.20$

0.036

0.602

\section{(IV)}

$\mathrm{C}_{25} \mathrm{H}_{22} \mathrm{O}_{2}$

354.42

Triclinic, $P \overline{1}$

9.2870 (2), 9.8727 (2), 12.2944 (3)

67.098 (3), 81.472 (2), 61.989 (3)

$915.92(5)$

$\mathrm{Cu} K \alpha$

$0.17 \times 0.11 \times 0.04$

$0.034,0.092,1.04 \quad 0.032,0.080,1.07$

$\mathrm{H}$-atom parameters constrained $\quad \mathrm{H}$-atom parameters constrained chi single CCD 2017)

$0.781,1.000$

$29818,3336,3073$

$0.19,-0.16$
XtaLAB AFC11 (RCD3): quarter-

Gaussian (CrysAlis PRO; Rigaku,

\begin{tabular}{|c|c|c|}
\hline & (IV) & (V) \\
\hline \multicolumn{3}{|l|}{ Crystal data } \\
\hline Chemical formula & $\mathrm{C}_{18} \mathrm{H}_{15} \mathrm{ClO}$ & $\mathrm{C}_{19} \mathrm{H}_{15} \mathrm{NO}$ \\
\hline$M_{\mathrm{r}}$ & 282.75 & 273.32 \\
\hline Crystal system, space group & Monoclinic, $P 2_{1} / n$ & Monoclinic, $P 2_{1} / c$ \\
\hline Temperature $(\mathrm{K})$ & 100 & 100 \\
\hline$a, b, c(\AA)$ & $10.6273(5), 11.6191(4), 12.1114(5)$ & $12.4725(4), 7.1718(2), 15.9983(5)$ \\
\hline$\alpha, \beta, \gamma\left({ }^{\circ}\right)$ & $90,108.777$ (4), 90 & $90,106.120(3), 90$ \\
\hline$V\left(\AA^{3}\right)$ & $1415.92(11)$ & $1374.79(8)$ \\
\hline$Z$ & 4 & 4 \\
\hline Radiation type & $\mathrm{Cu} K \alpha$ & $\mathrm{Cu} K \alpha$ \\
\hline$\mu\left(\mathrm{mm}^{-1}\right)$ & 2.31 & 0.64 \\
\hline Crystal size $(\mathrm{mm})$ & $0.28 \times 0.20 \times 0.03$ & $0.17 \times 0.10 \times 0.03$ \\
\hline \multicolumn{3}{|l|}{ Data collection } \\
\hline Diffractometer & $\begin{array}{l}\text { XtaLAB AFC11 (RCD3): quarter-chi single } \\
\text { CCD }\end{array}$ & $\begin{array}{l}\text { XtaLAB AFC11 (RCD3): quarter-chi single } \\
\text { CCD }\end{array}$ \\
\hline Absorption correction & Multi-scan (CrysAlis PRO; Rigaku, 2017) & Gaussian (CrysAlis PRO; Rigaku, 2017) \\
\hline$T_{\min }, T_{\max }$ & $0.722,1.000$ & $0.895,1.000$ \\
\hline $\begin{array}{l}\text { No. of measured, independent and observed } \\
{[I>2 \sigma(I)] \text { reflections }}\end{array}$ & $11747,2568,2203$ & $9732,2511,2302$ \\
\hline$R_{\text {int }}$ & 0.073 & 0.059 \\
\hline$(\sin \theta / \lambda)_{\max }\left(\AA^{-1}\right)$ & 0.602 & 0.602 \\
\hline \multicolumn{3}{|l|}{ Refinement } \\
\hline$R\left[F^{2}>2 \sigma\left(F^{2}\right)\right], w R\left(F^{2}\right), S$ & $0.055,0.165,1.11$ & $0.068,0.181,1.06$ \\
\hline No. of reflections & 2568 & 2511 \\
\hline No. of parameters & 181 & 190 \\
\hline $\mathrm{H}$-atom treatment & $\mathrm{H}$-atom parameters constrained & $\mathrm{H}$-atom parameters constrained \\
\hline$\Delta \rho_{\max }, \Delta \rho_{\min }\left(\mathrm{e} \AA^{-3}\right)$ & $0.32,-0.41$ & $0.49,-0.32$ \\
\hline
\end{tabular}

Computer programs: CrysAlis PRO (Rigaku, 2017), SHELXS97 (Sheldrick, 2008), SHELXL2014 (Sheldrick, 2015), ORTEP-3 (Farrugia, 2012) and publCIF (Westrip, 2010).

Recrystallization from ethanol solution at room temperature yielded colourless blocks [(I), (III) and (V)] and plates [(II) and (IV)]. Spectroscopic data for (I)-(V) are available as supporting information. 


\section{Refinement}

Crystal data, data collection and structure refinement details are summarized in Table 8. All $\mathrm{H}$ atoms were located geometrically $(\mathrm{C}-\mathrm{H}=0.95-0.99 \AA)$ and refined as riding atoms, with $U_{\text {iso }}(\mathrm{H})=1.2 U_{\text {eq }}(\mathrm{C})$ or $1.5 U_{\text {eq }}$ (methyl C). The methyl groups in (I) and (II) were allowed to rotate, but not to tip, to best fit the electron density.

\section{Acknowledgements}

We thank the EPSRC National Crystallography Service (University of Southampton) for the X-ray data collections and Edward Tiekink for assistance with the overlay plot.

\section{References}

Al-Nakib, T. M., Perjesi, P., Varghese, R. \& Meegan, M. J. (1997). Med. Princ. Pract. 6, 14-21.

Baddeley, T. C., Gomes, L. R., Low, J. N., Turner, A. B., Wardell, J. L. \& Watson, G. J. R. (2017a). Z. Kristallogr. 232, 317-334.

Baddeley, T. C., Gomes, L. R., Low, J. N., Turner, A. B., Wardell, J. L. \& Watson, G. J. R. (2017b). Z. Kristallogr 232, 697-718.

Dimmock, J. R., Kandepu, N. M., Nazarali, A. J., Kowalchuk, T. P., Motaganahalli, N., Quail, J. W., Mykytiuk, P., Audette, G. F., Prasad, L., Perjési, P., Allen, T. M., Santos, C. L., Szydlowski, J., De Clercq, E. \& Balzarini, J. (1999). J. Med. Chem. 42, 1358-1366.

Dimmock, J. R., Zello, G. A., Oloo, E. O., Quail, J. W., Kraatz, H.-B., Perjési, P., Aradi, F., Takács-Novák, K., Allen, T. M., Santos, C. L., Balzarini, J., De Clercq, E. \& Stables, J. P. (2002). J. Med. Chem. 45, 3103-3111.
Farrugia, L. J. (2012). J. Appl. Cryst. 45, 849-854.

Fodor, K., Tomescova, V., Kőszegi, T., Kron, I. \& Perjési, P. (2011). Monatsh. Chem. 142, 463-468.

Gans, J. \& Shalloway, D. (2001). J. Mol. Graph. Model. 19, 557-9, 609.

Gautam, Y., Dwivedi, S., Srivastava, A., Hamidullah, H., Singh, A., Chanda, D., Singh, J., Rai, S., Konwar, R. \& Negi, A. S. (2016). RSC Adv. 6, 33369-33379.

Groom, C. R., Bruno, I. J., Lightfoot, M. P. \& Ward, S. C. (2016). Acta Cryst. B72, 171-179.

Gupta, D. \& Jain, D. K. (2015). J. Adv. Pharm. Technol. Res. 6, 114117.

Kitaigorodskii, A. I. (1961). In Organic Chemical Crystallography. New York: Consultants Bureau.

Lo Presti, L. (2018). CrystEngComm, 20, 5976-5989.

Rigaku (2017). CrysAlis PRO. Rigaku Corporation, Tokyo, Japan.

Sheldrick, G. M. (2008). Acta Cryst. A64, 112-122.

Sheldrick, G. M. (2015). Acta Cryst. C71, 3-8.

Spek, A. L. (2009). Acta Cryst. D65, 148-155.

Tan, S. L., Jotani, M. M. \& Tiekink, E. R. T. (2019). Acta Cryst. E75, 308-318.

Turner, M. J., McKinnon, J. J., Wolff, S. K., Grimwood, D. J., Spackman, P. R., Jayatilaka, D. \& Spackman, M. A. (2017). CrystalExplorer17. University of Western Australia. http://hirshfeldsurface.net.

Watson, G. J. R., Turner, A. B. \& Allen, S. (1993). Organic Materials for Non-linear Optics III, edited by G. J. Ashwell \& D. Bloor. RSC Special Publication No. 137, pp. 112-117. Cambridge: Royal Society of Chemistry.

Westrip, S. P. (2010). J. Appl. Cryst. 43, 920-925. 


\section{supporting information}

Acta Cryst. (2019). E75, 1741-1747 [https://doi.org/10.1107/S2056989019014245]

Different packing motifs mediated by weak interactions and polymorphism in the crystal structures of five 2-(benzylidene)benzosuberone derivatives

Lewis S. Seaman, Cristiane F. da Costa, Marcus V. N. de Souza, Solange M. S. V. Wardell, James

L. Wardell and William T. A. Harrison

Computing details

For all structures, data collection: CrysAlis PRO (Rigaku, 2017); cell refinement: CrysAlis PRO (Rigaku, 2017); data reduction: CrysAlis PRO (Rigaku, 2017); program(s) used to solve structure: SHELXS97 (Sheldrick, 2008); program(s) used to refine structure: SHELXL2014 (Sheldrick, 2015); molecular graphics: ORTEP-3 (Farrugia, 2012); software used to prepare material for publication: publCIF (Westrip, 2010).

6-(4-Methoxybenzylidene)-6,7,8,9-tetrahydro-5H-benzo[7]annulen-5-one (I)

Crystal data

$\mathrm{C}_{19} \mathrm{H}_{18} \mathrm{O}_{2}$

$M_{r}=278.33$

Monoclinic, $P 2_{1} / c$

$a=10.9171$ (3) $\AA$

$b=9.1262(2) \AA$

$c=15.2539(3) \AA$

$\beta=108.618(3)^{\circ}$

$V=1440.24(6) \AA^{3}$

$Z=4$

Data collection

XtaLAB AFC12 (RCD3): Kappa single CCD diffractometer

Radiation source: Rotating-anode X-ray tube

Mirror monochromator

$\omega$ scans

Absorption correction: multi-scan

(CrysAlis PRO; Rigaku, 2017)

$T_{\min }=0.877, T_{\max }=1.000$

Refinement

Refinement on $F^{2}$

Least-squares matrix: full

$R\left[F^{2}>2 \sigma\left(F^{2}\right)\right]=0.037$

$w R\left(F^{2}\right)=0.093$

$S=1.03$

3296 reflections

191 parameters

0 restraints
$F(000)=592$

$D_{\mathrm{x}}=1.284 \mathrm{Mg} \mathrm{m}^{-3}$

Mo $K \alpha$ radiation, $\lambda=0.71073 \AA$

Cell parameters from 6994 reflections

$\theta=3.6-30.6^{\circ}$

$\mu=0.08 \mathrm{~mm}^{-1}$

$T=100 \mathrm{~K}$

Block, colourless

$0.20 \times 0.15 \times 0.05 \mathrm{~mm}$

16988 measured reflections

3296 independent reflections

2843 reflections with $I>2 \sigma(I)$

$R_{\text {int }}=0.033$

$\theta_{\text {max }}=27.5^{\circ}, \theta_{\text {min }}=2.6^{\circ}$

$h=-13 \rightarrow 14$

$k=-11 \rightarrow 11$

$l=-19 \rightarrow 19$

Primary atom site location: structure-invariant direct methods

Hydrogen site location: inferred from neighbouring sites

$\mathrm{H}$-atom parameters constrained

$w=1 /\left[\sigma^{2}\left(F_{\mathrm{o}}{ }^{2}\right)+(0.0435 P)^{2}+0.435 P\right]$

where $P=\left(F_{\mathrm{o}}^{2}+2 F_{\mathrm{c}}{ }^{2}\right) / 3$

$(\Delta / \sigma)_{\max }<0.001$ 
$\Delta \rho_{\max }=0.25$ e $\AA^{-3}$

$\Delta \rho_{\min }=-0.18$ e $\AA^{-3}$

Special details

Geometry. All esds (except the esd in the dihedral angle between two 1.s. planes) are estimated using the full covariance matrix. The cell esds are taken into account individually in the estimation of esds in distances, angles and torsion angles; correlations between esds in cell parameters are only used when they are defined by crystal symmetry. An approximate (isotropic) treatment of cell esds is used for estimating esds involving l.s. planes.

Fractional atomic coordinates and isotropic or equivalent isotropic displacement parameters $\left(\hat{A}^{2}\right)$

\begin{tabular}{|c|c|c|c|c|}
\hline & $x$ & $y$ & $z$ & $U_{\text {iso }} * / U_{\text {eq }}$ \\
\hline $\mathrm{C} 1$ & $0.21223(10)$ & $0.67551(12)$ & $0.33073(7)$ & $0.0185(2)$ \\
\hline $\mathrm{C} 2$ & $0.25289(10)$ & $0.51984(12)$ & $0.35285(7)$ & $0.0176(2)$ \\
\hline $\mathrm{C} 3$ & $0.26700(10)$ & $0.42493(12)$ & $0.27568(7)$ & $0.0192(2)$ \\
\hline $\mathrm{H} 3 \mathrm{~A}$ & 0.3201 & 0.3384 & 0.3032 & $0.023^{*}$ \\
\hline $\mathrm{H} 3 \mathrm{~B}$ & 0.3146 & 0.4809 & 0.2413 & $0.023^{*}$ \\
\hline $\mathrm{C} 4$ & 0.13905 (11) & $0.37140(12)$ & $0.20672(7)$ & $0.0235(2)$ \\
\hline $\mathrm{H} 4 \mathrm{~A}$ & 0.1543 & 0.3392 & 0.1491 & $0.028^{*}$ \\
\hline $\mathrm{H} 4 \mathrm{~B}$ & 0.1077 & 0.2858 & 0.2333 & $0.028^{*}$ \\
\hline $\mathrm{C} 5$ & 0.03505 (11) & $0.49088(12)$ & $0.18348(8)$ & $0.0228(2)$ \\
\hline $\mathrm{H} 5 \mathrm{~A}$ & -0.0381 & 0.4586 & 0.1296 & $0.027^{*}$ \\
\hline $\mathrm{H} 5 \mathrm{~B}$ & 0.0022 & 0.5024 & 0.2365 & $0.027^{*}$ \\
\hline C6 & $0.08193(10)$ & $0.63762(12)$ & $0.16175(7)$ & $0.0187(2)$ \\
\hline $\mathrm{C} 7$ & 0.03877 (11) & 0.69424 (13) & $0.07205(7)$ & $0.0220(2)$ \\
\hline $\mathrm{H} 7$ & -0.0170 & 0.6369 & 0.0237 & $0.026^{*}$ \\
\hline $\mathrm{C} 8$ & 0.07549 (11) & $0.83208(13)$ & $0.05212(8)$ & $0.0237(2)$ \\
\hline $\mathrm{H} 8$ & 0.0461 & 0.8677 & -0.0096 & $0.028^{*}$ \\
\hline $\mathrm{C} 9$ & 0.15527 (11) & $0.91857(13)$ & $0.12212(8)$ & $0.0227(2)$ \\
\hline H9 & 0.1795 & 1.0139 & 0.1087 & $0.027 *$ \\
\hline $\mathrm{C} 10$ & $0.19928(10)$ & $0.86472(12)$ & $0.21178(7)$ & $0.0199(2)$ \\
\hline $\mathrm{H} 10$ & 0.2534 & 0.9237 & 0.2600 & $0.024^{*}$ \\
\hline C11 & $0.16467(10)$ & $0.72442(12)$ & $0.23167(7)$ & $0.0178(2)$ \\
\hline $\mathrm{C} 12$ & $0.28541(10)$ & $0.48266(12)$ & $0.44276(7)$ & $0.0184(2)$ \\
\hline H12 & 0.2785 & 0.5598 & 0.4828 & $0.022 *$ \\
\hline $\mathrm{C} 13$ & $0.32958(10)$ & $0.34296(12)$ & $0.48884(7)$ & $0.0179(2)$ \\
\hline $\mathrm{C} 14$ & $0.28868(10)$ & $0.20616(12)$ & $0.44938(7)$ & $0.0186(2)$ \\
\hline H14 & 0.2300 & 0.2016 & 0.3881 & $0.022 *$ \\
\hline $\mathrm{C} 15$ & $0.33124(10)$ & $0.07653(12)$ & $0.49704(7)$ & $0.0192(2)$ \\
\hline H15 & 0.3014 & -0.0152 & 0.4689 & $0.023^{*}$ \\
\hline $\mathrm{C} 16$ & $0.41828(10)$ & $0.08253(12)$ & $0.58673(7)$ & $0.0182(2)$ \\
\hline $\mathrm{C} 17$ & $0.45955(10)$ & $0.21776(12)$ & $0.62786(7)$ & $0.0205(2)$ \\
\hline H17 & 0.5189 & 0.2220 & 0.6889 & $0.025^{*}$ \\
\hline $\mathrm{C} 18$ & $0.41448(11)$ & $0.34552(12)$ & $0.58016(7)$ & $0.0204(2)$ \\
\hline H18 & 0.4414 & 0.4371 & 0.6096 & $0.024^{*}$ \\
\hline C19 & $0.41639(11)$ & $-0.17646(12)$ & $0.60092(8)$ & $0.0220(2)$ \\
\hline H19A & 0.3224 & -0.1777 & 0.5874 & $0.033^{*}$ \\
\hline H19B & 0.4555 & -0.2544 & 0.6452 & $0.033^{*}$ \\
\hline H19C & 0.4370 & -0.1924 & 0.5436 & $0.033^{*}$ \\
\hline $\mathrm{O} 1$ & $0.21902(8)$ & $0.76586(9)$ & $0.39153(5)$ & $0.0253(2)$ \\
\hline
\end{tabular}




$\begin{array}{lllll}\mathrm{O} 2 & 0.46646(7) & -0.03768(8) & 0.63967(5) & 0.02142(18)\end{array}$

Atomic displacement parameters $\left(\AA^{2}\right)$

\begin{tabular}{lllllll}
\hline & $U^{11}$ & $U^{22}$ & $U^{33}$ & $U^{12}$ & $U^{13}$ & $U^{23}$ \\
\hline C1 & $0.0175(5)$ & $0.0198(5)$ & $0.0189(5)$ & $-0.0002(4)$ & $0.0067(4)$ & $-0.0003(4)$ \\
C2 & $0.0171(5)$ & $0.0181(5)$ & $0.0178(5)$ & $-0.0003(4)$ & $0.0058(4)$ & $-0.0001(4)$ \\
C3 & $0.0236(6)$ & $0.0186(5)$ & $0.0166(5)$ & $0.0020(4)$ & $0.0081(4)$ & $0.0007(4)$ \\
C4 & $0.0305(6)$ & $0.0199(6)$ & $0.0183(5)$ & $-0.0024(5)$ & $0.0054(5)$ & $-0.0002(4)$ \\
C5 & $0.0215(6)$ & $0.0247(6)$ & $0.0204(5)$ & $-0.0040(4)$ & $0.0041(4)$ & $0.0002(4)$ \\
C6 & $0.0165(5)$ & $0.0211(5)$ & $0.0192(5)$ & $0.0020(4)$ & $0.0065(4)$ & $0.0011(4)$ \\
C7 & $0.0195(5)$ & $0.0265(6)$ & $0.0186(5)$ & $0.0036(4)$ & $0.0041(4)$ & $0.0004(4)$ \\
C8 & $0.0246(6)$ & $0.0283(6)$ & $0.0192(5)$ & $0.0083(5)$ & $0.0083(4)$ & $0.0070(4)$ \\
C9 & $0.0250(6)$ & $0.0203(6)$ & $0.0260(6)$ & $0.0048(4)$ & $0.0127(5)$ & $0.0057(4)$ \\
C10 & $0.0196(5)$ & $0.0194(5)$ & $0.0220(5)$ & $0.0027(4)$ & $0.0086(4)$ & $-0.0001(4)$ \\
C11 & $0.0174(5)$ & $0.0192(5)$ & $0.0182(5)$ & $0.0032(4)$ & $0.0076(4)$ & $0.0015(4)$ \\
C12 & $0.0187(5)$ & $0.0193(5)$ & $0.0181(5)$ & $0.0003(4)$ & $0.0070(4)$ & $-0.0014(4)$ \\
C13 & $0.0177(5)$ & $0.0213(5)$ & $0.0159(5)$ & $0.0009(4)$ & $0.0071(4)$ & $0.0008(4)$ \\
C14 & $0.0178(5)$ & $0.0233(6)$ & $0.0142(5)$ & $-0.0003(4)$ & $0.0042(4)$ & $0.0008(4)$ \\
C15 & $0.0200(5)$ & $0.0204(5)$ & $0.0175(5)$ & $-0.0015(4)$ & $0.0065(4)$ & $-0.0002(4)$ \\
C16 & $0.0179(5)$ & $0.0218(5)$ & $0.0163(5)$ & $0.0027(4)$ & $0.0076(4)$ & $0.0037(4)$ \\
C17 & $0.0193(5)$ & $0.0271(6)$ & $0.0140(5)$ & $0.0004(4)$ & $0.0037(4)$ & $0.0003(4)$ \\
C18 & $0.0222(5)$ & $0.0218(5)$ & $0.0176(5)$ & $-0.0011(4)$ & $0.0071(4)$ & $-0.0024(4)$ \\
C19 & $0.0241(6)$ & $0.0199(5)$ & $0.0226(5)$ & $0.0015(4)$ & $0.0081(4)$ & $0.0041(4)$ \\
O1 & $0.0349(5)$ & $0.0208(4)$ & $0.0187(4)$ & $0.0036(3)$ & $0.0066(3)$ & $-0.0019(3)$ \\
O2 & $0.0239(4)$ & $0.0209(4)$ & $0.0177(4)$ & $0.0025(3)$ & $0.0043(3)$ & $0.0037(3)$ \\
& & & & & & \\
& & & & & &
\end{tabular}

Geometric parameters $\left(\AA,{ }^{\circ}\right)$

\begin{tabular}{llll}
\hline $\mathrm{C} 1-\mathrm{O} 1$ & $1.2254(13)$ & $\mathrm{C} 9-\mathrm{H} 9$ & 0.9500 \\
$\mathrm{C} 1-\mathrm{C} 2$ & $1.4945(15)$ & $\mathrm{C} 10-\mathrm{C} 11$ & $1.3955(15)$ \\
$\mathrm{C} 1-\mathrm{C} 11$ & $1.5003(14)$ & $\mathrm{C} 10-\mathrm{H} 10$ & 0.9500 \\
$\mathrm{C} 2-\mathrm{C} 12$ & $1.3458(15)$ & $\mathrm{C} 12-\mathrm{C} 13$ & $1.4614(15)$ \\
$\mathrm{C} 2-\mathrm{C} 3$ & $1.5085(14)$ & $\mathrm{C} 12-\mathrm{H} 12$ & 0.9500 \\
$\mathrm{C} 3-\mathrm{C} 4$ & $1.5356(15)$ & $\mathrm{C} 13-\mathrm{C} 14$ & $1.3959(15)$ \\
$\mathrm{C} 3-\mathrm{H} 3 \mathrm{~A}$ & 0.9900 & $\mathrm{C} 13-\mathrm{C} 18$ & $1.4056(15)$ \\
$\mathrm{C} 3-\mathrm{H} 3 \mathrm{~B}$ & 0.9900 & $\mathrm{C} 14-\mathrm{C} 15$ & $1.3887(15)$ \\
$\mathrm{C} 4-\mathrm{C} 5$ & $1.5319(16)$ & $\mathrm{C} 14-\mathrm{H} 14$ & 0.9500 \\
$\mathrm{C} 4-\mathrm{H} 4 \mathrm{~A}$ & 0.9900 & $\mathrm{C} 15-\mathrm{C} 16$ & $1.3956(15)$ \\
$\mathrm{C} 4-\mathrm{H} 4 \mathrm{~B}$ & 0.9900 & $\mathrm{C} 15-\mathrm{H} 15$ & 1.9500 \\
$\mathrm{C} 5-\mathrm{C} 6$ & $1.5078(15)$ & $\mathrm{C} 16-\mathrm{O} 2$ & $1.3642(12)$ \\
$\mathrm{C} 5-\mathrm{H} 5 \mathrm{~A}$ & 0.9900 & $\mathrm{C} 16-\mathrm{C} 17$ & $1.3793(15)$ \\
$\mathrm{C} 5-\mathrm{H} 5 \mathrm{~B}$ & 0.9900 & $\mathrm{C} 17-\mathrm{C} 18$ & 0.9500 \\
$\mathrm{C} 6-\mathrm{C} 7$ & $1.3962(15)$ & $\mathrm{C} 17-\mathrm{H} 17$ & 0.9500 \\
$\mathrm{C} 6-\mathrm{C} 11$ & $1.4021(15)$ & $\mathrm{C} 18-\mathrm{H} 18$ & $1.4304(13)$ \\
$\mathrm{C} 7-\mathrm{C} 8$ & $1.3832(16)$ & $\mathrm{C} 19-\mathrm{O} 2$ & 0.9800 \\
$\mathrm{C} 7-\mathrm{H} 7$ & 0.9500 & $\mathrm{C} 19-\mathrm{H} 19 \mathrm{~A}$ & 0.9800 \\
$\mathrm{C} 8-\mathrm{C} 9$ & $1.3885(17)$ & $\mathrm{C} 19-\mathrm{H} 19 \mathrm{~B}$ &
\end{tabular}




\begin{tabular}{|c|c|c|c|}
\hline $\begin{array}{l}\mathrm{C} 8-\mathrm{H} 8 \\
\mathrm{C} 9-\mathrm{C} 10\end{array}$ & $\begin{array}{l}0.9500 \\
1.3869(15)\end{array}$ & $\mathrm{C} 19-\mathrm{H} 19 \mathrm{C}$ & 0.9800 \\
\hline $\mathrm{O} 1-\mathrm{C} 1-\mathrm{C} 2$ & $121.79(10)$ & $\mathrm{C} 8-\mathrm{C} 9-\mathrm{H} 9$ & 120.3 \\
\hline $\mathrm{O} 1-\mathrm{C} 1-\mathrm{C} 11$ & $118.68(10)$ & $\mathrm{C} 9-\mathrm{C} 10-\mathrm{C} 11$ & $120.47(11)$ \\
\hline $\mathrm{C} 2-\mathrm{C} 1-\mathrm{C} 11$ & $119.51(9)$ & $\mathrm{C} 9-\mathrm{C} 10-\mathrm{H} 10$ & 119.8 \\
\hline $\mathrm{C} 12-\mathrm{C} 2-\mathrm{C} 1$ & $115.63(9)$ & $\mathrm{C} 11-\mathrm{C} 10-\mathrm{H} 10$ & 119.8 \\
\hline $\mathrm{C} 12-\mathrm{C} 2-\mathrm{C} 3$ & $126.23(10)$ & $\mathrm{C} 10-\mathrm{C} 11-\mathrm{C} 6$ & $120.42(10)$ \\
\hline $\mathrm{C} 1-\mathrm{C} 2-\mathrm{C} 3$ & $117.81(9)$ & $\mathrm{C} 10-\mathrm{C} 11-\mathrm{C} 1$ & $117.47(10)$ \\
\hline $\mathrm{C} 2-\mathrm{C} 3-\mathrm{C} 4$ & $114.83(9)$ & $\mathrm{C} 6-\mathrm{C} 11-\mathrm{C} 1$ & $121.99(9)$ \\
\hline $\mathrm{C} 2-\mathrm{C} 3-\mathrm{H} 3 \mathrm{~A}$ & 108.6 & $\mathrm{C} 2-\mathrm{C} 12-\mathrm{C} 13$ & $130.49(10)$ \\
\hline $\mathrm{C} 4-\mathrm{C} 3-\mathrm{H} 3 \mathrm{~A}$ & 108.6 & $\mathrm{C} 2-\mathrm{C} 12-\mathrm{H} 12$ & 114.8 \\
\hline $\mathrm{C} 2-\mathrm{C} 3-\mathrm{H} 3 \mathrm{~B}$ & 108.6 & $\mathrm{C} 13-\mathrm{C} 12-\mathrm{H} 12$ & 114.8 \\
\hline $\mathrm{C} 4-\mathrm{C} 3-\mathrm{H} 3 \mathrm{~B}$ & 108.6 & $\mathrm{C} 14-\mathrm{C} 13-\mathrm{C} 18$ & $117.46(10)$ \\
\hline $\mathrm{H} 3 \mathrm{~A}-\mathrm{C} 3-\mathrm{H} 3 \mathrm{~B}$ & 107.5 & $\mathrm{C} 14-\mathrm{C} 13-\mathrm{C} 12$ & $124.18(9)$ \\
\hline $\mathrm{C} 5-\mathrm{C} 4-\mathrm{C} 3$ & $112.15(9)$ & $\mathrm{C} 18-\mathrm{C} 13-\mathrm{C} 12$ & $118.30(10)$ \\
\hline $\mathrm{C} 5-\mathrm{C} 4-\mathrm{H} 4 \mathrm{~A}$ & 109.2 & $\mathrm{C} 15-\mathrm{C} 14-\mathrm{C} 13$ & $121.92(10)$ \\
\hline $\mathrm{C} 3-\mathrm{C} 4-\mathrm{H} 4 \mathrm{~A}$ & 109.2 & $\mathrm{C} 15-\mathrm{C} 14-\mathrm{H} 14$ & 119.0 \\
\hline $\mathrm{C} 5-\mathrm{C} 4-\mathrm{H} 4 \mathrm{~B}$ & 109.2 & $\mathrm{C} 13-\mathrm{C} 14-\mathrm{H} 14$ & 119.0 \\
\hline $\mathrm{C} 3-\mathrm{C} 4-\mathrm{H} 4 \mathrm{~B}$ & 109.2 & $\mathrm{C} 14-\mathrm{C} 15-\mathrm{C} 16$ & $119.28(10)$ \\
\hline $\mathrm{H} 4 \mathrm{~A}-\mathrm{C} 4-\mathrm{H} 4 \mathrm{~B}$ & 107.9 & $\mathrm{C} 14-\mathrm{C} 15-\mathrm{H} 15$ & 120.4 \\
\hline $\mathrm{C} 6-\mathrm{C} 5-\mathrm{C} 4$ & $113.86(9)$ & $\mathrm{C} 16-\mathrm{C} 15-\mathrm{H} 15$ & 120.4 \\
\hline $\mathrm{C} 6-\mathrm{C} 5-\mathrm{H} 5 \mathrm{~A}$ & 108.8 & $\mathrm{O} 2-\mathrm{C} 16-\mathrm{C} 17$ & $115.97(9)$ \\
\hline $\mathrm{C} 4-\mathrm{C} 5-\mathrm{H} 5 \mathrm{~A}$ & 108.8 & $\mathrm{O} 2-\mathrm{C} 16-\mathrm{C} 15$ & $124.19(10)$ \\
\hline $\mathrm{C} 6-\mathrm{C} 5-\mathrm{H} 5 \mathrm{~B}$ & 108.8 & $\mathrm{C} 17-\mathrm{C} 16-\mathrm{C} 15$ & $119.84(10)$ \\
\hline $\mathrm{C} 4-\mathrm{C} 5-\mathrm{H} 5 \mathrm{~B}$ & 108.8 & $\mathrm{C} 18-\mathrm{C} 17-\mathrm{C} 16$ & $120.12(10)$ \\
\hline $\mathrm{H} 5 \mathrm{~A}-\mathrm{C} 5-\mathrm{H} 5 \mathrm{~B}$ & 107.7 & $\mathrm{C} 18-\mathrm{C} 17-\mathrm{H} 17$ & 119.9 \\
\hline $\mathrm{C} 7-\mathrm{C} 6-\mathrm{C} 11$ & $118.05(10)$ & $\mathrm{C} 16-\mathrm{C} 17-\mathrm{H} 17$ & 119.9 \\
\hline $\mathrm{C} 7-\mathrm{C} 6-\mathrm{C} 5$ & $120.80(10)$ & $\mathrm{C} 17-\mathrm{C} 18-\mathrm{C} 13$ & $121.34(10)$ \\
\hline $\mathrm{C} 11-\mathrm{C} 6-\mathrm{C} 5$ & $121.07(9)$ & $\mathrm{C} 17-\mathrm{C} 18-\mathrm{H} 18$ & 119.3 \\
\hline $\mathrm{C} 8-\mathrm{C} 7-\mathrm{C} 6$ & $121.41(11)$ & $\mathrm{C} 13-\mathrm{C} 18-\mathrm{H} 18$ & 119.3 \\
\hline $\mathrm{C} 8-\mathrm{C} 7-\mathrm{H} 7$ & 119.3 & $\mathrm{O} 2-\mathrm{C} 19-\mathrm{H} 19 \mathrm{~A}$ & 109.5 \\
\hline $\mathrm{C} 6-\mathrm{C} 7-\mathrm{H} 7$ & 119.3 & $\mathrm{O} 2-\mathrm{C} 19-\mathrm{H} 19 \mathrm{~B}$ & 109.5 \\
\hline $\mathrm{C} 7-\mathrm{C} 8-\mathrm{C} 9$ & $120.18(10)$ & $\mathrm{H} 19 \mathrm{~A}-\mathrm{C} 19-\mathrm{H} 19 \mathrm{~B}$ & 109.5 \\
\hline $\mathrm{C} 7-\mathrm{C} 8-\mathrm{H} 8$ & 119.9 & $\mathrm{O} 2-\mathrm{C} 19-\mathrm{H} 19 \mathrm{C}$ & 109.5 \\
\hline $\mathrm{C} 9-\mathrm{C} 8-\mathrm{H} 8$ & 119.9 & $\mathrm{H} 19 \mathrm{~A}-\mathrm{C} 19-\mathrm{H} 19 \mathrm{C}$ & 109.5 \\
\hline $\mathrm{C} 10-\mathrm{C} 9-\mathrm{C} 8$ & $119.45(11)$ & $\mathrm{H} 19 \mathrm{~B}-\mathrm{C} 19-\mathrm{H} 19 \mathrm{C}$ & 109.5 \\
\hline $\mathrm{C} 10-\mathrm{C} 9-\mathrm{H} 9$ & 120.3 & $\mathrm{C} 16-\mathrm{O} 2-\mathrm{C} 19$ & $116.31(8)$ \\
\hline $\mathrm{O} 1-\mathrm{C} 1-\mathrm{C} 2-\mathrm{C} 12$ & $6.07(15)$ & $\mathrm{O} 1-\mathrm{C} 1-\mathrm{C} 11-\mathrm{C} 10$ & $36.06(14)$ \\
\hline $\mathrm{C} 11-\mathrm{C} 1-\mathrm{C} 2-\mathrm{C} 12$ & $-175.42(9)$ & $\mathrm{C} 2-\mathrm{C} 1-\mathrm{C} 11-\mathrm{C} 10$ & $-142.50(10)$ \\
\hline $\mathrm{O} 1-\mathrm{C} 1-\mathrm{C} 2-\mathrm{C} 3$ & $-167.73(10)$ & $\mathrm{O} 1-\mathrm{C} 1-\mathrm{C} 11-\mathrm{C} 6$ & $-140.03(11)$ \\
\hline $\mathrm{C} 11-\mathrm{C} 1-\mathrm{C} 2-\mathrm{C} 3$ & $10.78(14)$ & $\mathrm{C} 2-\mathrm{C} 1-\mathrm{C} 11-\mathrm{C} 6$ & $41.42(14)$ \\
\hline $\mathrm{C} 12-\mathrm{C} 2-\mathrm{C} 3-\mathrm{C} 4$ & $110.47(12)$ & $\mathrm{C} 1-\mathrm{C} 2-\mathrm{C} 12-\mathrm{C} 13$ & $-179.67(10)$ \\
\hline $\mathrm{C} 1-\mathrm{C} 2-\mathrm{C} 3-\mathrm{C} 4$ & $-76.47(12)$ & $\mathrm{C} 3-\mathrm{C} 2-\mathrm{C} 12-\mathrm{C} 13$ & $-6.47(19)$ \\
\hline $\mathrm{C} 2-\mathrm{C} 3-\mathrm{C} 4-\mathrm{C} 5$ & $40.48(13)$ & $\mathrm{C} 2-\mathrm{C} 12-\mathrm{C} 13-\mathrm{C} 14$ & $-33.81(17)$ \\
\hline $\mathrm{C} 3-\mathrm{C} 4-\mathrm{C} 5-\mathrm{C} 6$ & $46.53(12)$ & $\mathrm{C} 2-\mathrm{C} 12-\mathrm{C} 13-\mathrm{C} 18$ & $148.98(11)$ \\
\hline $\mathrm{C} 4-\mathrm{C} 5-\mathrm{C} 6-\mathrm{C} 7$ & $110.60(11)$ & $\mathrm{C} 18-\mathrm{C} 13-\mathrm{C} 14-\mathrm{C} 15$ & $-1.16(15)$ \\
\hline
\end{tabular}




$\begin{array}{llll}\mathrm{C} 4-\mathrm{C} 5-\mathrm{C} 6-\mathrm{C} 11 & -72.74(13) & \mathrm{C} 12-\mathrm{C} 13-\mathrm{C} 14-\mathrm{C} 15 & -178.38(10) \\ \mathrm{C} 11-\mathrm{C} 6-\mathrm{C} 7-\mathrm{C} 8 & -0.30(16) & \mathrm{C} 13-\mathrm{C} 14-\mathrm{C} 15-\mathrm{C} 16 & -0.55(15) \\ \mathrm{C} 5-\mathrm{C} 6-\mathrm{C} 7-\mathrm{C} 8 & 176.46(10) & \mathrm{C} 14-\mathrm{C} 15-\mathrm{C} 16-\mathrm{O} 2 & -179.87(9) \\ \mathrm{C} 6-\mathrm{C} 7-\mathrm{C} 8-\mathrm{C} 9 & \mathrm{C} 14-\mathrm{C} 15-\mathrm{C} 16-\mathrm{C} 17 & 1.11(15) \\ \mathrm{C} 7-\mathrm{C} 8-\mathrm{C} 9-\mathrm{C} 10 & 0.97(16) & \mathrm{O} 2-\mathrm{C} 16-\mathrm{C} 17-\mathrm{C} 18 & -179.03(9) \\ \mathrm{C} 8-\mathrm{C} 9-\mathrm{C} 10-\mathrm{C} 11 & 0.46(16) & \mathrm{C} 15-\mathrm{C} 16-\mathrm{C} 17-\mathrm{C} 18 & 0.07(15) \\ \mathrm{C} 9-\mathrm{C} 10-\mathrm{C} 11-\mathrm{C} 6 & -1.83(15) & \mathrm{C} 16-\mathrm{C} 17-\mathrm{C} 18-\mathrm{C} 13 & -1.85(16) \\ \mathrm{C} 9-\mathrm{C} 10-\mathrm{C} 11-\mathrm{C} 1 & -177.98(9) & \mathrm{C} 14-\mathrm{C} 13-\mathrm{C} 18-\mathrm{C} 17 & 2.36(15) \\ \mathrm{C} 7-\mathrm{C} 6-\mathrm{C} 11-\mathrm{C} 10 & 1.73(15) & \mathrm{C} 12-\mathrm{C} 13-\mathrm{C} 18-\mathrm{C} 17 & 179.75(9) \\ \mathrm{C} 5-\mathrm{C} 6-\mathrm{C} 11-\mathrm{C} 10 & -175.02(10) & \mathrm{C} 17-\mathrm{C} 16-\mathrm{O} 2-\mathrm{C} 19 & 174.98(9) \\ \mathrm{C} 7-\mathrm{C} 6-\mathrm{C} 11-\mathrm{C} 1 & 177.70(9) & \mathrm{C} 15-\mathrm{C} 16-\mathrm{O} 2-\mathrm{C} 19 & -4.07(14) \\ \mathrm{C} 5-\mathrm{C} 6-\mathrm{C} 11-\mathrm{C} 1 & 0.95(15) & & \end{array}$

Hydrogen-bond geometry $\left(\AA,{ }^{\circ}\right)$

\begin{tabular}{lllll}
\hline$D-\mathrm{H} \cdots A$ & $D-\mathrm{H}$ & $\mathrm{H} \cdots A$ & $D \cdots A$ & $D-\mathrm{H} \cdots A$ \\
\hline $\mathrm{C} 15-\mathrm{H} 15 \cdots \mathrm{O} 1^{\mathrm{i}}$ & 0.95 & 2.35 & $3.2971(14)$ & 176 \\
$\mathrm{C} 19-\mathrm{H} 19 A \cdots C g 1^{\mathrm{ii}}$ & 0.98 & 2.76 & $3.6165(13)$ & 146 \\
$\mathrm{C} 19-\mathrm{H} 19 C \cdots C g 2^{\mathrm{iii}}$ & 0.98 & 2.74 & $3.6029(13)$ & 147 \\
\hline
\end{tabular}

Symmetry codes: (i) $x, y-1, z$; (ii) $x,-y+1 / 2, z+1 / 2$; (iii) $-x+1,-y,-z+1$.

6-(4-Ethoxybenzylidene)-6,7,8,9-tetrahydro-5H-benzo[7]annulen-5-one (II)

\section{Crystal data}

$\mathrm{C}_{20} \mathrm{H}_{20} \mathrm{O}_{2}$

$M_{r}=292.36$

Monoclinic, $P 2_{1} / c$

$a=12.6208(2) \AA$

$b=14.99690$ (17) $\AA$

$c=8.39151$ (12) $\AA$

$\beta=108.6814(17)^{\circ}$

$V=1504.60(4) \AA^{3}$

$Z=4$

\section{Data collection}

XtaLAB AFC11 (RCD3): quarter-chi single CCD diffractometer

Radiation source: Rotating-anode X-ray tube Mirror monochromator

$\omega$ scans

Absorption correction: gaussian

(CrysAlis PRO; Rigaku, 2017)

$T_{\min }=0.772, T_{\max }=1.000$

\section{Refinement}

Refinement on $F^{2}$

Least-squares matrix: full

$R\left[F^{2}>2 \sigma\left(F^{2}\right)\right]=0.034$

$w R\left(F^{2}\right)=0.092$

$S=1.04$

2704 reflections
$F(000)=624$

$D_{\mathrm{x}}=1.291 \mathrm{Mg} \mathrm{m}^{-3}$

$\mathrm{Cu} K \alpha$ radiation, $\lambda=1.54184 \AA$

Cell parameters from 4578 reflections

$\theta=3.7-69.7^{\circ}$

$\mu=0.64 \mathrm{~mm}^{-1}$

$T=100 \mathrm{~K}$

Plate, colourless

$0.20 \times 0.11 \times 0.03 \mathrm{~mm}$

9197 measured reflections

2704 independent reflections

2486 reflections with $I>2 \sigma(I)$

$R_{\text {int }}=0.024$

$\theta_{\text {max }}=68.3^{\circ}, \theta_{\min }=3.7^{\circ}$

$h=-14 \rightarrow 15$

$k=-18 \rightarrow 12$

$l=-9 \rightarrow 9$

\section{1 parameters}

0 restraints

Primary atom site location: structure-invariant direct methods

Hydrogen site location: inferred from neighbouring sites 
H-atom parameters constrained

$w=1 /\left[\sigma^{2}\left(F_{\mathrm{o}}^{2}\right)+(0.0491 P)^{2}+0.4554 P\right]$

where $P=\left(F_{\mathrm{o}}^{2}+2 F_{\mathrm{c}}^{2}\right) / 3$

$(\Delta / \sigma)_{\max }<0.001$

$\Delta \rho_{\max }=0.25$ e $\AA^{-3}$
$\Delta \rho_{\min }=-0.20$ e $\AA^{-3}$

Extinction correction: SHELXL2014

(Sheldrick, 2015),

$\mathrm{Fc}^{*}=\mathrm{kFc}\left[1+0.001 \mathrm{xFc}^{2} \lambda^{3} / \sin (2 \theta)\right]^{-1 / 4}$

Extinction coefficient: 0.0019 (3)

Special details

Geometry. All esds (except the esd in the dihedral angle between two 1.s. planes) are estimated using the full covariance matrix. The cell esds are taken into account individually in the estimation of esds in distances, angles and torsion angles; correlations between esds in cell parameters are only used when they are defined by crystal symmetry. An approximate (isotropic) treatment of cell esds is used for estimating esds involving l.s. planes.

Fractional atomic coordinates and isotropic or equivalent isotropic displacement parameters $\left(\AA^{2}\right)$

\begin{tabular}{|c|c|c|c|c|}
\hline & $x$ & $y$ & $z$ & $U_{\text {iso }} * / U_{\text {eq }}$ \\
\hline $\mathrm{C} 1$ & $0.29882(10)$ & $0.41642(7)$ & $0.43555(14)$ & 0.0187 (3) \\
\hline $\mathrm{C} 2$ & $0.34088(9)$ & $0.37739(8)$ & $0.60870(14)$ & 0.0181 \\
\hline C3 & $0.27266(9)$ & $0.30342(8)$ & $0.64912(14)$ & 0.0183 \\
\hline $\mathrm{H} 3 \mathrm{~A}$ & 0.3219 & 0.2668 & 0.7414 & $0.022 *$ \\
\hline H3B & 0.2438 & 0.2646 & 0.5492 & $0.022 *$ \\
\hline $\mathrm{C} 4$ & $0.17406(9)$ & $0.33720(8)$ & $0.70118(14)$ & 0.0196 \\
\hline $\mathrm{H} 4 \mathrm{~A}$ & 0.2021 & 0.3603 & 0.8178 & $0.024^{*}$ \\
\hline H4B & 0.1226 & 0.2870 & 0.6990 & $0.024 *$ \\
\hline $\mathrm{C} 5$ & $0.10987(10)$ & $0.41143(8)$ & $0.58327(14)$ & 0.0203 \\
\hline $\mathrm{H} 5 \mathrm{~A}$ & 0.0363 & 0.4193 & 0.6001 & $0.024^{*}$ \\
\hline H5B & 0.1517 & 0.4680 & 0.6155 & $0.024 *$ \\
\hline C6 & $0.09109(10)$ & $0.39437(7)$ & $0.39855(14)$ & 0.0187 \\
\hline $\mathrm{C} 7$ & $-0.01602(10)$ & $0.37634(8)$ & $0.28964(15)$ & 0.0218 \\
\hline $\mathrm{H7}$ & -0.0757 & 0.3683 & 0.3342 & $0.026^{*}$ \\
\hline $\mathrm{C} 8$ & $-0.03708(10)$ & $0.36985(8)$ & $0.11691(15)$ & $0.0239(3)$ \\
\hline H8 & -0.1104 & 0.3564 & 0.0451 & $0.029 *$ \\
\hline $\mathrm{C} 9$ & $0.04853(10)$ & $0.38295(8)$ & $0.04913(15)$ & 0.0233 \\
\hline H9 & 0.0337 & 0.3808 & -0.0692 & $0.028 *$ \\
\hline $\mathrm{C} 10$ & $0.15569(10)$ & $0.39914(8)$ & $0.15573(14)$ & $0.0201(3)$ \\
\hline $\mathrm{H} 10$ & 0.2145 & 0.4080 & 0.1097 & $0.024 *$ \\
\hline C11 & $0.17889(10)$ & $0.40265(7)$ & $0.33013(14)$ & 0.0183 \\
\hline $\mathrm{C} 12$ & $0.43722(9)$ & $0.41219(8)$ & 0.71077 (14) & $0.0182(3)$ \\
\hline H12 & 0.4688 & 0.4565 & 0.6587 & $0.022 *$ \\
\hline $\mathrm{C} 13$ & $0.50252(10)$ & $0.39471(7)$ & $0.88640(14)$ & 0.0183 \\
\hline $\mathrm{C} 14$ & $0.46487(10)$ & $0.35195(8)$ & $1.00672(15)$ & 0.0207 \\
\hline H14 & 0.3911 & 0.3284 & 0.9737 & $0.025 *$ \\
\hline C15 & $0.53262(10)$ & $0.34296(8)$ & $1.17341(15)$ & 0.0209 \\
\hline H15 & 0.5052 & 0.3134 & 1.2523 & $0.025^{*}$ \\
\hline C16 & $0.64063(10)$ & $0.37742(7)$ & $1.22393(14)$ & $0.0186(3)$ \\
\hline $\mathrm{C} 17$ & $0.67988(9)$ & $0.42064(8)$ & $1.10640(14)$ & 0.0193 \\
\hline H17 & 0.7537 & 0.4441 & 1.1399 & $0.023^{*}$ \\
\hline $\mathrm{C} 18$ & $0.61171(10)$ & $0.42937(8)$ & $0.94187(14)$ & $0.0186(3)$ \\
\hline H18 & 0.6393 & 0.4597 & 0.8639 & $0.022 *$ \\
\hline C19 & $0.67659(10)$ & $0.33225(8)$ & $1.51117(15)$ & 0.0229 \\
\hline
\end{tabular}




\begin{tabular}{lllll}
$\mathrm{H} 19 \mathrm{~A}$ & 0.6531 & 0.2700 & 1.4798 & $0.028^{*}$ \\
$\mathrm{H} 19 \mathrm{~B}$ & 0.6122 & 0.3655 & 1.5247 & $0.028^{*}$ \\
$\mathrm{C} 20$ & $0.77366(11)$ & $0.33441(9)$ & $1.67216(15)$ & $0.0271(3)$ \\
$\mathrm{H} 20 \mathrm{~A}$ & 0.7515 & 0.3069 & 1.7626 & $0.041^{*}$ \\
$\mathrm{H} 20 \mathrm{~B}$ & 0.7960 & 0.3964 & 1.7018 & $0.041^{*}$ \\
$\mathrm{H} 20 \mathrm{C}$ & 0.8367 & 0.3014 & 1.6569 & $0.041^{*}$ \\
$\mathrm{O} 1$ & $0.36138(7)$ & $0.45674(6)$ & $0.37458(10)$ & $0.0264(2)$ \\
$\mathrm{O} 2$ & $0.71428(7)$ & $0.37337(6)$ & $1.38349(10)$ & $0.0217(2)$ \\
\hline
\end{tabular}

Atomic displacement parameters $\left(\AA^{2}\right)$

\begin{tabular}{lllllll}
\hline & $U^{11}$ & $U^{22}$ & $U^{33}$ & $U^{12}$ & $U^{13}$ & $U^{23}$ \\
\hline C1 & $0.0219(6)$ & $0.0195(6)$ & $0.0159(6)$ & $-0.0012(4)$ & $0.0076(5)$ & $-0.0007(4)$ \\
C2 & $0.0192(6)$ & $0.0212(6)$ & $0.0155(6)$ & $0.0017(4)$ & $0.0077(5)$ & $0.0005(4)$ \\
C3 & $0.0182(6)$ & $0.0208(6)$ & $0.0155(5)$ & $-0.0006(4)$ & $0.0049(4)$ & $0.0011(4)$ \\
C4 & $0.0195(6)$ & $0.0250(6)$ & $0.0154(5)$ & $-0.0014(5)$ & $0.0071(5)$ & $0.0005(4)$ \\
C5 & $0.0196(6)$ & $0.0251(6)$ & $0.0171(6)$ & $0.0020(5)$ & $0.0072(5)$ & $-0.0011(4)$ \\
C6 & $0.0204(6)$ & $0.0187(5)$ & $0.0171(6)$ & $0.0027(4)$ & $0.0059(5)$ & $0.0012(4)$ \\
C7 & $0.0203(6)$ & $0.0255(6)$ & $0.0203(6)$ & $0.0027(5)$ & $0.0073(5)$ & $-0.0004(5)$ \\
C8 & $0.0197(6)$ & $0.0297(6)$ & $0.0195(6)$ & $0.0019(5)$ & $0.0023(5)$ & $-0.0015(5)$ \\
C9 & $0.0260(6)$ & $0.0279(6)$ & $0.0144(6)$ & $0.0027(5)$ & $0.0045(5)$ & $-0.0007(5)$ \\
C10 & $0.0233(6)$ & $0.0213(6)$ & $0.0169(6)$ & $0.0008(5)$ & $0.0081(5)$ & $0.0007(4)$ \\
C11 & $0.0205(6)$ & $0.0173(5)$ & $0.0167(6)$ & $0.0004(4)$ & $0.0056(5)$ & $0.0002(4)$ \\
C12 & $0.0184(6)$ & $0.0207(6)$ & $0.0176(6)$ & $0.0010(4)$ & $0.0087(5)$ & $0.0007(4)$ \\
C13 & $0.0188(6)$ & $0.0198(5)$ & $0.0170(6)$ & $0.0010(4)$ & $0.0067(5)$ & $-0.0008(4)$ \\
C14 & $0.0169(6)$ & $0.0258(6)$ & $0.0195(6)$ & $-0.0022(5)$ & $0.0063(5)$ & $0.0001(5)$ \\
C15 & $0.0216(6)$ & $0.0254(6)$ & $0.0175(6)$ & $-0.0014(5)$ & $0.0090(5)$ & $0.0022(4)$ \\
C16 & $0.0202(6)$ & $0.0200(6)$ & $0.0149(6)$ & $0.0026(4)$ & $0.0049(5)$ & $-0.0009(4)$ \\
C17 & $0.0174(6)$ & $0.0220(6)$ & $0.0187(6)$ & $-0.0008(4)$ & $0.0060(5)$ & $-0.0012(4)$ \\
C18 & $0.0205(6)$ & $0.0199(6)$ & $0.0173(6)$ & $0.0003(4)$ & $0.0089(5)$ & $0.0002(4)$ \\
C19 & $0.0277(6)$ & $0.0260(6)$ & $0.0160(6)$ & $-0.0015(5)$ & $0.0084(5)$ & $0.0017(5)$ \\
C20 & $0.0329(7)$ & $0.0287(7)$ & $0.0174(6)$ & $-0.0010(5)$ & $0.0049(5)$ & $0.0016(5)$ \\
O1 & $0.0244(5)$ & $0.0366(5)$ & $0.0183(4)$ & $-0.0081(4)$ & $0.0071(3)$ & $0.0043(4)$ \\
O2 & $0.0207(4)$ & $0.0296(5)$ & $0.0137(4)$ & $-0.0018(3)$ & $0.0041(3)$ & $0.0025(3)$ \\
& & & & & & \\
\hline & & & & & & \\
\hline
\end{tabular}

Geometric parameters $\left(\AA,{ }^{\circ}\right)$

\begin{tabular}{llll}
\hline $\mathrm{C} 1-\mathrm{O} 1$ & $1.2286(14)$ & $\mathrm{C} 10-\mathrm{C} 11$ & $1.3985(16)$ \\
$\mathrm{C} 1-\mathrm{C} 2$ & $1.4971(16)$ & $\mathrm{C} 10-\mathrm{H} 10$ & 0.9500 \\
$\mathrm{C} 1-\mathrm{C} 11$ & $1.5027(16)$ & $\mathrm{C} 12-\mathrm{C} 13$ & $1.4635(16)$ \\
$\mathrm{C} 2-\mathrm{C} 12$ & $1.3481(17)$ & $\mathrm{C} 12-\mathrm{H} 12$ & 0.9500 \\
$\mathrm{C} 2-\mathrm{C} 3$ & $1.5079(16)$ & $\mathrm{C} 13-\mathrm{C} 14$ & $1.4014(16)$ \\
$\mathrm{C} 3-\mathrm{C} 4$ & $1.5311(15)$ & $\mathrm{C} 13-\mathrm{C} 18$ & $1.4053(17)$ \\
$\mathrm{C} 3-\mathrm{H} 3 \mathrm{~A}$ & 0.9900 & $\mathrm{C} 14-\mathrm{C} 15$ & $1.3927(16)$ \\
$\mathrm{C} 3-\mathrm{H} 3 \mathrm{~B}$ & 0.9900 & $\mathrm{C} 14-\mathrm{H} 14$ & 0.9500 \\
$\mathrm{C} 4-\mathrm{C} 5$ & $1.5365(16)$ & $\mathrm{C} 15-\mathrm{C} 16$ & $1.3909(17)$ \\
$\mathrm{C} 4-\mathrm{H} 4 \mathrm{~A}$ & 0.9900 & $\mathrm{C} 15-\mathrm{H} 15$ & 0.9500 \\
$\mathrm{C} 4-\mathrm{H} 4 \mathrm{~B}$ & 0.9900 & $\mathrm{C} 16-\mathrm{O} 2$ & $1.3650(14)$
\end{tabular}




\begin{tabular}{|c|c|c|c|}
\hline $\mathrm{C} 5-\mathrm{C} 6$ & $1.5130(16)$ & $\mathrm{C} 16-\mathrm{C} 17$ & $1.3968(16)$ \\
\hline $\mathrm{C} 5-\mathrm{H} 5 \mathrm{~A}$ & 0.9900 & $\mathrm{C} 17-\mathrm{C} 18$ & $1.3794(16)$ \\
\hline C5-H5B & 0.9900 & C17-H17 & 0.9500 \\
\hline $\mathrm{C} 6-\mathrm{C} 7$ & $1.3944(17)$ & $\mathrm{C} 18-\mathrm{H} 18$ & 0.9500 \\
\hline $\mathrm{C} 6-\mathrm{C} 11$ & $1.4074(16)$ & $\mathrm{C} 19-\mathrm{O} 2$ & $1.4426(14)$ \\
\hline $\mathrm{C} 7-\mathrm{C} 8$ & $1.3908(17)$ & $\mathrm{C} 19-\mathrm{C} 20$ & $1.5056(17)$ \\
\hline $\mathrm{C} 7-\mathrm{H} 7$ & 0.9500 & C19-H19A & 0.9900 \\
\hline $\mathrm{C} 8-\mathrm{C} 9$ & $1.3868(18)$ & C19-H19B & 0.9900 \\
\hline $\mathrm{C} 8-\mathrm{H} 8$ & 0.9500 & $\mathrm{C} 20-\mathrm{H} 20 \mathrm{~A}$ & 0.9800 \\
\hline $\mathrm{C} 9-\mathrm{C} 10$ & $1.3832(18)$ & $\mathrm{C} 20-\mathrm{H} 20 \mathrm{~B}$ & 0.9800 \\
\hline C9-H9 & 0.9500 & $\mathrm{C} 20-\mathrm{H} 20 \mathrm{C}$ & 0.9800 \\
\hline $\mathrm{O} 1-\mathrm{C} 1-\mathrm{C} 2$ & $121.41(10)$ & $\mathrm{C} 11-\mathrm{C} 10-\mathrm{H} 10$ & 119.4 \\
\hline $\mathrm{O} 1-\mathrm{C} 1-\mathrm{C} 11$ & $118.88(10)$ & $\mathrm{C} 10-\mathrm{C} 11-\mathrm{C} 6$ & $119.69(11)$ \\
\hline $\mathrm{C} 2-\mathrm{C} 1-\mathrm{C} 11$ & $119.65(10)$ & $\mathrm{C} 10-\mathrm{C} 11-\mathrm{C} 1$ & $117.07(10)$ \\
\hline $\mathrm{C} 12-\mathrm{C} 2-\mathrm{C} 1$ & $115.63(10)$ & $\mathrm{C} 6-\mathrm{C} 11-\mathrm{C} 1$ & $123.24(10)$ \\
\hline $\mathrm{C} 12-\mathrm{C} 2-\mathrm{C} 3$ & $127.28(10)$ & $\mathrm{C} 2-\mathrm{C} 12-\mathrm{C} 13$ & $132.29(11)$ \\
\hline $\mathrm{C} 1-\mathrm{C} 2-\mathrm{C} 3$ & $117.09(10)$ & $\mathrm{C} 2-\mathrm{C} 12-\mathrm{H} 12$ & 113.9 \\
\hline $\mathrm{C} 2-\mathrm{C} 3-\mathrm{C} 4$ & $113.30(9)$ & $\mathrm{C} 13-\mathrm{C} 12-\mathrm{H} 12$ & 113.9 \\
\hline $\mathrm{C} 2-\mathrm{C} 3-\mathrm{H} 3 \mathrm{~A}$ & 108.9 & $\mathrm{C} 14-\mathrm{C} 13-\mathrm{C} 18$ & $116.96(11)$ \\
\hline $\mathrm{C} 4-\mathrm{C} 3-\mathrm{H} 3 \mathrm{~A}$ & 108.9 & $\mathrm{C} 14-\mathrm{C} 13-\mathrm{C} 12$ & $126.59(11)$ \\
\hline $\mathrm{C} 2-\mathrm{C} 3-\mathrm{H} 3 \mathrm{~B}$ & 108.9 & $\mathrm{C} 18-\mathrm{C} 13-\mathrm{C} 12$ & $116.32(10)$ \\
\hline $\mathrm{C} 4-\mathrm{C} 3-\mathrm{H} 3 \mathrm{~B}$ & 108.9 & $\mathrm{C} 15-\mathrm{C} 14-\mathrm{C} 13$ & $121.81(11)$ \\
\hline $\mathrm{H} 3 \mathrm{~A}-\mathrm{C} 3-\mathrm{H} 3 \mathrm{~B}$ & 107.7 & $\mathrm{C} 15-\mathrm{C} 14-\mathrm{H} 14$ & 119.1 \\
\hline $\mathrm{C} 3-\mathrm{C} 4-\mathrm{C} 5$ & $111.41(9)$ & $\mathrm{C} 13-\mathrm{C} 14-\mathrm{H} 14$ & 119.1 \\
\hline $\mathrm{C} 3-\mathrm{C} 4-\mathrm{H} 4 \mathrm{~A}$ & 109.3 & $\mathrm{C} 16-\mathrm{C} 15-\mathrm{C} 14$ & $119.77(11)$ \\
\hline $\mathrm{C} 5-\mathrm{C} 4-\mathrm{H} 4 \mathrm{~A}$ & 109.3 & $\mathrm{C} 16-\mathrm{C} 15-\mathrm{H} 15$ & 120.1 \\
\hline $\mathrm{C} 3-\mathrm{C} 4-\mathrm{H} 4 \mathrm{~B}$ & 109.3 & $\mathrm{C} 14-\mathrm{C} 15-\mathrm{H} 15$ & 120.1 \\
\hline $\mathrm{C} 5-\mathrm{C} 4-\mathrm{H} 4 \mathrm{~B}$ & 109.3 & $\mathrm{O} 2-\mathrm{C} 16-\mathrm{C} 15$ & $125.13(10)$ \\
\hline $\mathrm{H} 4 \mathrm{~A}-\mathrm{C} 4-\mathrm{H} 4 \mathrm{~B}$ & 108.0 & $\mathrm{O} 2-\mathrm{C} 16-\mathrm{C} 17$ & $115.41(10)$ \\
\hline $\mathrm{C} 6-\mathrm{C} 5-\mathrm{C} 4$ & $114.49(10)$ & $\mathrm{C} 15-\mathrm{C} 16-\mathrm{C} 17$ & $119.46(11)$ \\
\hline $\mathrm{C} 6-\mathrm{C} 5-\mathrm{H} 5 \mathrm{~A}$ & 108.6 & $\mathrm{C} 18-\mathrm{C} 17-\mathrm{C} 16$ & $120.15(11)$ \\
\hline $\mathrm{C} 4-\mathrm{C} 5-\mathrm{H} 5 \mathrm{~A}$ & 108.6 & $\mathrm{C} 18-\mathrm{C} 17-\mathrm{H} 17$ & 119.9 \\
\hline $\mathrm{C} 6-\mathrm{C} 5-\mathrm{H} 5 \mathrm{~B}$ & 108.6 & $\mathrm{C} 16-\mathrm{C} 17-\mathrm{H} 17$ & 119.9 \\
\hline $\mathrm{C} 4-\mathrm{C} 5-\mathrm{H} 5 \mathrm{~B}$ & 108.6 & $\mathrm{C} 17-\mathrm{C} 18-\mathrm{C} 13$ & $121.83(10)$ \\
\hline $\mathrm{H} 5 \mathrm{~A}-\mathrm{C} 5-\mathrm{H} 5 \mathrm{~B}$ & 107.6 & $\mathrm{C} 17-\mathrm{C} 18-\mathrm{H} 18$ & 119.1 \\
\hline $\mathrm{C} 7-\mathrm{C} 6-\mathrm{C} 11$ & $118.32(11)$ & $\mathrm{C} 13-\mathrm{C} 18-\mathrm{H} 18$ & 119.1 \\
\hline $\mathrm{C} 7-\mathrm{C} 6-\mathrm{C} 5$ & $120.34(11)$ & $\mathrm{O} 2-\mathrm{C} 19-\mathrm{C} 20$ & $106.86(10)$ \\
\hline $\mathrm{C} 11-\mathrm{C} 6-\mathrm{C} 5$ & $121.17(10)$ & $\mathrm{O} 2-\mathrm{C} 19-\mathrm{H} 19 \mathrm{~A}$ & 110.3 \\
\hline $\mathrm{C} 8-\mathrm{C} 7-\mathrm{C} 6$ & $121.21(11)$ & $\mathrm{C} 20-\mathrm{C} 19-\mathrm{H} 19 \mathrm{~A}$ & 110.3 \\
\hline $\mathrm{C} 8-\mathrm{C} 7-\mathrm{H} 7$ & 119.4 & $\mathrm{O} 2-\mathrm{C} 19-\mathrm{H} 19 \mathrm{~B}$ & 110.3 \\
\hline $\mathrm{C} 6-\mathrm{C} 7-\mathrm{H} 7$ & 119.4 & $\mathrm{C} 20-\mathrm{C} 19-\mathrm{H} 19 \mathrm{~B}$ & 110.3 \\
\hline $\mathrm{C} 9-\mathrm{C} 8-\mathrm{C} 7$ & $120.24(11)$ & $\mathrm{H} 19 \mathrm{~A}-\mathrm{C} 19-\mathrm{H} 19 \mathrm{~B}$ & 108.6 \\
\hline $\mathrm{C} 9-\mathrm{C} 8-\mathrm{H} 8$ & 119.9 & $\mathrm{C} 19-\mathrm{C} 20-\mathrm{H} 20 \mathrm{~A}$ & 109.5 \\
\hline $\mathrm{C} 7-\mathrm{C} 8-\mathrm{H} 8$ & 119.9 & $\mathrm{C} 19-\mathrm{C} 20-\mathrm{H} 20 \mathrm{~B}$ & 109.5 \\
\hline $\mathrm{C} 10-\mathrm{C} 9-\mathrm{C} 8$ & $119.24(11)$ & $\mathrm{H} 20 \mathrm{~A}-\mathrm{C} 20-\mathrm{H} 20 \mathrm{~B}$ & 109.5 \\
\hline $\mathrm{C} 10-\mathrm{C} 9-\mathrm{H} 9$ & 120.4 & $\mathrm{C} 19-\mathrm{C} 20-\mathrm{H} 20 \mathrm{C}$ & 109.5 \\
\hline $\mathrm{C} 8-\mathrm{C} 9-\mathrm{H} 9$ & 120.4 & $\mathrm{H} 20 \mathrm{~A}-\mathrm{C} 20-\mathrm{H} 20 \mathrm{C}$ & 109.5 \\
\hline
\end{tabular}




$\begin{array}{ll}\mathrm{C} 9-\mathrm{C} 10-\mathrm{C} 11 & 121.14(11) \\ \mathrm{C} 9-\mathrm{C} 10-\mathrm{H} 10 & \\ \mathrm{O} 1-\mathrm{C} 1-\mathrm{C} 2-\mathrm{C} 12 & 20.55(16) \\ \mathrm{C} 11-\mathrm{C} 1-\mathrm{C} 2-\mathrm{C} 12 & -162.35(10) \\ \mathrm{O} 1-\mathrm{C} 1-\mathrm{C} 2-\mathrm{C} 3 & -158.66(11) \\ \mathrm{C} 11-\mathrm{C} 1-\mathrm{C} 2-\mathrm{C} 3 & 18.45(15) \\ \mathrm{C} 12-\mathrm{C} 2-\mathrm{C} 3-\mathrm{C} 4 & 97.96(14) \\ \mathrm{C} 1-\mathrm{C} 2-\mathrm{C} 3-\mathrm{C} 4 & -82.94(12) \\ \mathrm{C} 2-\mathrm{C} 3-\mathrm{C} 4-\mathrm{C} 5 & 45.35(13) \\ \mathrm{C} 3-\mathrm{C} 4-\mathrm{C} 5-\mathrm{C} 6 & 42.50(13) \\ \mathrm{C} 4-\mathrm{C} 5-\mathrm{C} 6-\mathrm{C} 7 & 110.32(12) \\ \mathrm{C} 4-\mathrm{C} 5-\mathrm{C} 6-\mathrm{C} 11 & -74.39(14) \\ \mathrm{C} 11-\mathrm{C} 6-\mathrm{C} 7-\mathrm{C} 8 & -2.33(17) \\ \mathrm{C} 5-\mathrm{C} 6-\mathrm{C} 7-\mathrm{C} 8 & 173.09(11) \\ \mathrm{C} 6-\mathrm{C} 7-\mathrm{C} 8-\mathrm{C} 9 & -1.20(18) \\ \mathrm{C} 7-\mathrm{C} 8-\mathrm{C} 9-\mathrm{C} 10 & 2.45(18) \\ \mathrm{C} 8-\mathrm{C} 9-\mathrm{C} 10-\mathrm{C} 11 & -0.12(18) \\ \mathrm{C} 9-\mathrm{C} 10-\mathrm{C} 11-\mathrm{C} 6 & -3.44(17) \\ \mathrm{C} 9-\mathrm{C} 10-\mathrm{C} 11-\mathrm{C} 1 & 177.26(11) \\ \mathrm{C} 7-\mathrm{C} 6-\mathrm{C} 11-\mathrm{C} 10 & 4.59(16) \\ \mathrm{C} 5-\mathrm{C} 6-\mathrm{C} 11-\mathrm{C} 10 & -170.79(10) \\ \mathrm{C} 7-\mathrm{C} 6-\mathrm{C} 11-\mathrm{C} 1 & -176.16(11) \\ \mathrm{C} 5-\mathrm{C} 6-\mathrm{C} 11-\mathrm{C} 1 & 8.46(17) \\ \end{array}$

$\mathrm{H} 20 \mathrm{~B}-\mathrm{C} 20-\mathrm{H} 20 \mathrm{C}$

$\mathrm{C} 16-\mathrm{O} 2-\mathrm{C} 19$

109.5

117.69 (9)

$27.78(16)$

$-149.40(11)$

$\mathrm{C} 2-\mathrm{C} 1-\mathrm{C} 11-\mathrm{C} 10$

$-151.50(11)$

$\mathrm{O} 1-\mathrm{C} 1-\mathrm{C} 11-\mathrm{C} 6$

$\mathrm{C} 2-\mathrm{C} 1-\mathrm{C} 11-\mathrm{C} 6$

$\mathrm{C} 1-\mathrm{C} 2-\mathrm{C} 12-\mathrm{C} 13$

$\mathrm{C} 3-\mathrm{C} 2-\mathrm{C} 12-\mathrm{C} 13$

$\mathrm{C} 2-\mathrm{C} 12-\mathrm{C} 13-\mathrm{C} 14$

$\mathrm{C} 2-\mathrm{C} 12-\mathrm{C} 13-\mathrm{C} 18$

$\mathrm{C} 18-\mathrm{C} 13-\mathrm{C} 14-\mathrm{C} 15$

$\mathrm{C} 12-\mathrm{C} 13-\mathrm{C} 14-\mathrm{C} 15$

$\mathrm{C} 13-\mathrm{C} 14-\mathrm{C} 15-\mathrm{C} 16$

$\mathrm{C} 14-\mathrm{C} 15-\mathrm{C} 16-\mathrm{O} 2$

$\mathrm{C} 14-\mathrm{C} 15-\mathrm{C} 16-\mathrm{C} 17$

$\mathrm{O} 2-\mathrm{C} 16-\mathrm{C} 17-\mathrm{C} 18$

$\mathrm{C} 15-\mathrm{C} 16-\mathrm{C} 17-\mathrm{C} 18$

C16-C17-C18-C13

$\mathrm{C} 14-\mathrm{C} 13-\mathrm{C} 18-\mathrm{C} 17$

$\mathrm{C} 12-\mathrm{C} 13-\mathrm{C} 18-\mathrm{C} 17$

$\mathrm{C} 15-\mathrm{C} 16-\mathrm{O} 2-\mathrm{C} 19$

$\mathrm{C} 17-\mathrm{C} 16-\mathrm{O} 2-\mathrm{C} 19$

$\mathrm{C} 20-\mathrm{C} 19-\mathrm{O} 2-\mathrm{C} 16$
31.33 (16)

$177.92(11)$

$-3.0(2)$

$-17.9(2)$

$166.41(12)$

-0.80 (17)

$-176.51(11)$

$0.32(18)$

$179.45(11)$

$-0.06(17)$

$-179.24(10)$

$0.32(17)$

$-0.85(17)$

1.07 (17)

$177.23(10)$

$-1.70(16)$

$177.83(10)$

$178.58(10)$

Hydrogen-bond geometry $\left(\AA,{ }^{\circ}\right)$

\begin{tabular}{lllll}
\hline$D-\mathrm{H} \cdots A$ & $D-\mathrm{H}$ & $\mathrm{H} \cdots A$ & $D \cdots A$ & $D-\mathrm{H} \cdots A$ \\
\hline $\mathrm{C} 18-\mathrm{H} 18 \cdots \mathrm{O} 1^{\mathrm{i}}$ & 0.95 & 2.36 & $3.2653(14)$ & 159 \\
$\mathrm{C} 4-\mathrm{H} 4 B \cdots C g 1^{\mathrm{ii}}$ & 0.98 & 2.72 & $3.6429(13)$ & 155 \\
$\mathrm{C} 19-\mathrm{H} 19 A \cdots C g 2^{\mathrm{ii}}$ & 0.98 & 2.71 & $3.5969(13)$ & 149 \\
\hline
\end{tabular}

Symmetry codes: (i) $-x+1,-y+1,-z+1$; (ii) $x,-y+1 / 2, z+1 / 2$.

2-(4-Benzylbenzylidene)-6,7,8,9-tetrahydro-5H-benzo[7]annulen-5-one (III)

Crystal data

$\mathrm{C}_{25} \mathrm{H}_{22} \mathrm{O}_{2}$

$M_{r}=354.42$

Triclinic, $P \overline{1}$

$a=9.2870(2) \AA$

$b=9.8727(2) \AA$

$c=12.2944$ (3) $\AA$

$\alpha=67.098(3)^{\circ}$

$\beta=81.472(2)^{\circ}$

$\gamma=61.989(3)^{\circ}$

$V=915.92(5) \AA^{3}$
$Z=2$

$F(000)=376$

$D_{\mathrm{x}}=1.285 \mathrm{Mg} \mathrm{m}^{-3}$

$\mathrm{Cu} K \alpha$ radiation, $\lambda=1.54184 \AA$

Cell parameters from 19041 reflections

$\theta=3.9-70.3^{\circ}$

$\mu=0.63 \mathrm{~mm}^{-1}$

$T=100 \mathrm{~K}$

Block, colourless

$0.17 \times 0.11 \times 0.04 \mathrm{~mm}$ 


\section{Data collection}

XtaLAB AFC11 (RCD3): quarter-chi single CCD diffractometer

Radiation source: Rotating-anode X-ray tube Mirror monochromator

$\omega$ scans

Absorption correction: gaussian

(CrysAlis PRO; Rigaku, 2017)

$T_{\min }=0.781, T_{\max }=1.000$

\section{Refinement}

Refinement on $F^{2}$

Least-squares matrix: full

$R\left[F^{2}>2 \sigma\left(F^{2}\right)\right]=0.032$

$w R\left(F^{2}\right)=0.080$

$S=1.07$

3336 reflections

245 parameters

0 restraints

Primary atom site location: structure-invariant direct methods
29818 measured reflections

3336 independent reflections

3073 reflections with $I>2 \sigma(I)$

$R_{\text {int }}=0.036$

$\theta_{\max }=68.2^{\circ}, \theta_{\min }=3.9^{\circ}$

$h=-11 \rightarrow 11$

$k=-11 \rightarrow 11$

$l=-14 \rightarrow 14$
Hydrogen site location: inferred from neighbouring sites

$\mathrm{H}$-atom parameters constrained

$w=1 /\left[\sigma^{2}\left(F_{\mathrm{o}}^{2}\right)+(0.0343 P)^{2}+0.2601 P\right]$

where $P=\left(F_{\mathrm{o}}^{2}+2 F_{\mathrm{c}}^{2}\right) / 3$

$(\Delta / \sigma)_{\max }<0.001$

$\Delta \rho_{\max }=0.19 \mathrm{e} \AA^{-3}$

$\Delta \rho_{\text {min }}=-0.16$ e $\AA^{-3}$

Extinction correction: SHELXL2014

(Sheldrick, 2015),

$\mathrm{Fc}^{*}=\mathrm{kFc}\left[1+0.001 \times \mathrm{Fc}^{2} \lambda^{3} / \sin (2 \theta)\right]^{-1 / 4}$

Extinction coefficient: 0.0027 (4)

\section{Special details}

Geometry. All esds (except the esd in the dihedral angle between two 1.s. planes) are estimated using the full covariance matrix. The cell esds are taken into account individually in the estimation of esds in distances, angles and torsion angles; correlations between esds in cell parameters are only used when they are defined by crystal symmetry. An approximate (isotropic) treatment of cell esds is used for estimating esds involving l.s. planes.

Fractional atomic coordinates and isotropic or equivalent isotropic displacement parameters $\left(\hat{A}^{2}\right)$

\begin{tabular}{lllll}
\hline & $x$ & $y$ & $z$ & $U_{\text {iso }}^{*} / U_{\text {eq }}$ \\
\hline C1 & $-0.05094(13)$ & $0.41786(13)$ & $0.37210(9)$ & $0.0206(2)$ \\
C2 & $0.11044(12)$ & $0.39278(12)$ & $0.40498(9)$ & $0.0201(2)$ \\
C3 & $0.15112(13)$ & $0.53459(13)$ & $0.34098(9)$ & $0.0218(2)$ \\
H3A & 0.2299 & 0.5293 & 0.3905 & $0.026^{*}$ \\
H3B & 0.0507 & 0.6397 & 0.3286 & $0.026^{*}$ \\
C4 & $0.22435(13)$ & $0.53175(14)$ & $0.22121(9)$ & $0.0253(2)$ \\
H4A & 0.3404 & 0.4482 & 0.2340 & $0.030^{*}$ \\
H4B & 0.2204 & 0.6406 & 0.1732 & $0.030^{*}$ \\
C5 & $0.13332(14)$ & $0.49174(14)$ & $0.15296(9)$ & $0.0261(2)$ \\
H5A & 0.1675 & 0.5174 & 0.0702 & $0.031^{*}$ \\
H5B & 0.1661 & 0.3720 & 0.1880 & $0.031^{*}$ \\
C6 & $-0.05030(13)$ & $0.58461(13)$ & $0.15292(9)$ & $0.0232(2)$ \\
C7 & $-0.14024(15)$ & $0.70291(13)$ & $0.04850(10)$ & $0.0286(3)$ \\
H7 & -0.0846 & 0.7283 & -0.0221 & $0.034^{*}$ \\
C8 & $-0.30954(15)$ & $0.78425(14)$ & $0.04574(10)$ & $0.0311(3)$ \\
H8 & -0.3685 & 0.8652 & -0.0262 & $0.037^{*}$ \\
C9 & $-0.39279(14)$ & $0.74770(14)$ & $0.14755(11)$ & $0.0295(3)$ \\
H9 & -0.5087 & 0.8033 & 0.1457 & $0.035^{*}$
\end{tabular}




$\begin{array}{lllll}\text { C10 } & -0.30610(13) & 0.62955(13) & 0.25218(10) & 0.0254(2) \\ \text { H10 } & -0.3632 & 0.6033 & 0.3218 & 0.030^{*} \\ \text { C11 } & -0.13575(13) & 0.54864(12) & 0.25643(9) & 0.0214(2) \\ \text { C12 } & 0.20322(12) & 0.24621(13) & 0.48767(9) & 0.0203(2) \\ \text { H12 } & 0.1549 & 0.1733 & 0.5162 & 0.024^{*} \\ \text { C13 } & 0.36457(13) & 0.17816(13) & 0.54185(9) & 0.0208(2) \\ \text { C14 } & 0.48036(13) & 0.23711(13) & 0.49726(9) & 0.0232(2) \\ \text { H14 } & 0.4568 & 0.3274 & 0.4241 & 0.028^{*} \\ \text { C15 } & 0.62886(13) & 0.16712(13) & 0.55709(9) & 0.0239(2) \\ \text { H15 } & 0.7051 & 0.2099 & 0.5253 & 0.029^{*} \\ \text { C16 } & 0.66527(12) & 0.03382(13) & 0.66400(9) & 0.0214(2) \\ \text { C17 } & 0.55458(13) & -0.03095(12) & 0.70815(9) & 0.0213(2) \\ \text { H17 } & 0.5800 & -0.1236 & 0.7799 & 0.026^{*} \\ \text { C18 } & 0.40818(13) & 0.03976(13) & 0.64743(9) & 0.0211(2) \\ \text { H18 } & 0.3344 & -0.0065 & 0.6779 & 0.025^{*} \\ \text { C19 } & 0.92156(13) & 0.02302(14) & 0.68813(10) & 0.0291(3) \\ \text { H19A } & 0.8709 & 0.1402 & 0.6798 & 0.035^{*} \\ \text { H19B } & 0.9567 & 0.0162 & 0.6096 & 0.035^{*} \\ \text { C20 } & 1.06575(13) & -0.07617(13) & 0.77387(9) & 0.0230(2) \\ \text { C21 } & 1.18414(13) & -0.22910(13) & 0.77295(10) & 0.0251(2) \\ \text { H21 } & 1.1719 & -0.2709 & 0.7190 & 0.030^{*} \\ \text { C22 } & 1.31964(13) & -0.32064(13) & 0.85007(10) & 0.0266(2) \\ \text { H22 } & 1.3985 & -0.4259 & 0.8499 & 0.032^{*} \\ \text { C23 } & 1.34081(13) & -0.25957(14) & 0.92737(10) & 0.0273(3) \\ \text { H23 } & 1.4350 & -0.3216 & 0.9791 & 0.033^{*} \\ \text { C24 } & 1.22388(14) & -0.10747(14) & 0.92885(10) & 0.0283(3) \\ \text { H24 } & 1.2376 & -0.0652 & 0.9819 & 0.034^{*} \\ \text { C25 } & 1.08673(13) & -0.01680(13) & 0.85305(10) & 0.0257(2) \\ \text { H25 } & 1.0063 & 0.0868 & 0.8552 & 0.031^{*} \\ \text { O1 } & -0.11776(9) & 0.33551(9) & 0.43769(6) & 0.02637(19) \\ \text { O2 } & 0.80574(9) & -0.04221(9) & 0.73220(6) & 0.02490(19)\end{array}$

Atomic displacement parameters $\left(\AA^{2}\right)$

\begin{tabular}{lllllll}
\hline & $U^{11}$ & $U^{22}$ & $U^{33}$ & $U^{12}$ & $U^{13}$ & $U^{23}$ \\
\hline C1 & $0.0216(5)$ & $0.0216(5)$ & $0.0211(5)$ & $-0.0107(4)$ & $0.0026(4)$ & $-0.0097(4)$ \\
C2 & $0.0206(5)$ & $0.0227(5)$ & $0.0199(5)$ & $-0.0118(4)$ & $0.0035(4)$ & $-0.0089(4)$ \\
C3 & $0.0214(5)$ & $0.0210(5)$ & $0.0235(5)$ & $-0.0110(4)$ & $0.0009(4)$ & $-0.0069(4)$ \\
C4 & $0.0233(6)$ & $0.0246(5)$ & $0.0255(6)$ & $-0.0122(5)$ & $0.0037(4)$ & $-0.0060(4)$ \\
C5 & $0.0296(6)$ & $0.0267(6)$ & $0.0219(5)$ & $-0.0135(5)$ & $0.0058(4)$ & $-0.0094(4)$ \\
C6 & $0.0298(6)$ & $0.0209(5)$ & $0.0224(5)$ & $-0.0128(5)$ & $0.0001(4)$ & $-0.0091(4)$ \\
C7 & $0.0407(7)$ & $0.0254(6)$ & $0.0226(6)$ & $-0.0172(5)$ & $-0.0016(5)$ & $-0.0077(5)$ \\
C8 & $0.0413(7)$ & $0.0218(6)$ & $0.0280(6)$ & $-0.0106(5)$ & $-0.0124(5)$ & $-0.0065(5)$ \\
C9 & $0.0269(6)$ & $0.0248(6)$ & $0.0362(6)$ & $-0.0062(5)$ & $-0.0092(5)$ & $-0.0140(5)$ \\
C10 & $0.0252(6)$ & $0.0254(6)$ & $0.0291(6)$ & $-0.0113(5)$ & $-0.0011(4)$ & $-0.0129(5)$ \\
C11 & $0.0240(5)$ & $0.0197(5)$ & $0.0232(5)$ & $-0.0101(4)$ & $-0.0012(4)$ & $-0.0096(4)$ \\
C12 & $0.0211(5)$ & $0.0232(5)$ & $0.0205(5)$ & $-0.0129(4)$ & $0.0036(4)$ & $-0.0091(4)$ \\
C13 & $0.0208(5)$ & $0.0212(5)$ & $0.0219(5)$ & $-0.0097(4)$ & $0.0017(4)$ & $-0.0092(4)$
\end{tabular}




$\begin{array}{lllllll}\text { C14 } & 0.0227(5) & 0.0230(5) & 0.0217(5) & -0.0114(4) & 0.0006(4) & -0.0046(4) \\ \text { C15 } & 0.0226(5) & 0.0256(5) & 0.0243(5) & -0.0144(5) & 0.0030(4) & -0.0063(4) \\ \text { C16 } & 0.0192(5) & 0.0221(5) & 0.0230(5) & -0.0088(4) & -0.0002(4) & -0.0086(4) \\ \text { C17 } & 0.0223(5) & 0.0197(5) & 0.0210(5) & -0.0105(4) & 0.0011(4) & -0.0055(4) \\ \text { C18 } & 0.0214(5) & 0.0217(5) & 0.0235(5) & -0.0122(4) & 0.0033(4) & -0.0092(4) \\ \text { C19 } & 0.0242(6) & 0.0305(6) & 0.0307(6) & -0.0183(5) & -0.0022(5) & -0.0008(5) \\ \text { C20 } & 0.0205(5) & 0.0242(5) & 0.0242(5) & -0.0146(4) & 0.0018(4) & -0.0033(4) \\ \text { C21 } & 0.0288(6) & 0.0261(6) & 0.0255(6) & -0.0174(5) & 0.0023(4) & -0.0088(4) \\ \text { C22 } & 0.0236(6) & 0.0217(5) & 0.0303(6) & -0.0103(5) & 0.0030(4) & -0.0062(5) \\ \text { C23 } & 0.0236(6) & 0.0296(6) & 0.0250(6) & -0.0153(5) & -0.0030(4) & -0.0009(5) \\ \text { C24 } & 0.0355(6) & 0.0325(6) & 0.0231(6) & -0.0209(5) & 0.0015(5) & -0.0093(5) \\ \text { C25 } & 0.0260(6) & 0.0224(5) & 0.0273(6) & -0.0115(5) & 0.0056(4) & -0.0085(4) \\ \text { O1 } & 0.0258(4) & 0.0314(4) & 0.0242(4) & -0.0182(3) & 0.0011(3) & -0.0058(3) \\ \text { O2 } & 0.0201(4) & 0.0269(4) & 0.0257(4) & -0.0141(3) & -0.0028(3) & -0.0019(3) \\ \end{array}$

Geometric parameters $\left(A,{ }^{\circ}\right)$

\begin{tabular}{|c|c|c|c|}
\hline $\mathrm{C} 1-\mathrm{O} 1$ & $1.2294(12)$ & $\mathrm{C} 13-\mathrm{C} 14$ & $1.4001(14)$ \\
\hline $\mathrm{C} 1-\mathrm{C} 2$ & $1.4914(14)$ & $\mathrm{C} 13-\mathrm{C} 18$ & $1.4042(15)$ \\
\hline $\mathrm{C} 1-\mathrm{C} 11$ & $1.5025(14)$ & $\mathrm{C} 14-\mathrm{C} 15$ & $1.3895(15)$ \\
\hline $\mathrm{C} 2-\mathrm{C} 12$ & $1.3467(15)$ & C14-H14 & 0.9500 \\
\hline $\mathrm{C} 2-\mathrm{C} 3$ & $1.5077(14)$ & $\mathrm{C} 15-\mathrm{C} 16$ & $1.3933(15)$ \\
\hline $\mathrm{C} 3-\mathrm{C} 4$ & $1.5326(15)$ & C15-H15 & 0.9500 \\
\hline $\mathrm{C} 3-\mathrm{H} 3 \mathrm{~A}$ & 0.9900 & $\mathrm{C} 16-\mathrm{O} 2$ & $1.3723(12)$ \\
\hline $\mathrm{C} 3-\mathrm{H} 3 \mathrm{~B}$ & 0.9900 & $\mathrm{C} 16-\mathrm{C} 17$ & $1.3936(14)$ \\
\hline $\mathrm{C} 4-\mathrm{C} 5$ & $1.5352(15)$ & $\mathrm{C} 17-\mathrm{C} 18$ & $1.3774(14)$ \\
\hline $\mathrm{C} 4-\mathrm{H} 4 \mathrm{~A}$ & 0.9900 & $\mathrm{C} 17-\mathrm{H} 17$ & 0.9500 \\
\hline $\mathrm{C} 4-\mathrm{H} 4 \mathrm{~B}$ & 0.9900 & $\mathrm{C} 18-\mathrm{H} 18$ & 0.9500 \\
\hline $\mathrm{C} 5-\mathrm{C} 6$ & $1.5099(15)$ & $\mathrm{C} 19-\mathrm{O} 2$ & $1.4392(12)$ \\
\hline $\mathrm{C} 5-\mathrm{H} 5 \mathrm{~A}$ & 0.9900 & $\mathrm{C} 19-\mathrm{C} 20$ & $1.4990(15)$ \\
\hline $\mathrm{C} 5-\mathrm{H} 5 \mathrm{~B}$ & 0.9900 & $\mathrm{C} 19-\mathrm{H} 19 \mathrm{~A}$ & 0.9900 \\
\hline $\mathrm{C} 6-\mathrm{C} 7$ & $1.3945(15)$ & C19-H19B & 0.9900 \\
\hline $\mathrm{C} 6-\mathrm{C} 11$ & $1.4102(15)$ & $\mathrm{C} 20-\mathrm{C} 25$ & $1.3916(16)$ \\
\hline $\mathrm{C} 7-\mathrm{C} 8$ & $1.3881(17)$ & $\mathrm{C} 20-\mathrm{C} 21$ & $1.3925(16)$ \\
\hline $\mathrm{C} 7-\mathrm{H} 7$ & 0.9500 & $\mathrm{C} 21-\mathrm{C} 22$ & $1.3849(16)$ \\
\hline $\mathrm{C} 8-\mathrm{C} 9$ & $1.3850(18)$ & $\mathrm{C} 21-\mathrm{H} 21$ & 0.9500 \\
\hline $\mathrm{C} 8-\mathrm{H} 8$ & 0.9500 & $\mathrm{C} 22-\mathrm{C} 23$ & $1.3850(16)$ \\
\hline $\mathrm{C} 9-\mathrm{C} 10$ & $1.3859(16)$ & $\mathrm{C} 22-\mathrm{H} 22$ & 0.9500 \\
\hline $\mathrm{C} 9-\mathrm{H} 9$ & 0.9500 & $\mathrm{C} 23-\mathrm{C} 24$ & $1.3850(17)$ \\
\hline $\mathrm{C} 10-\mathrm{C} 11$ & $1.3960(15)$ & $\mathrm{C} 23-\mathrm{H} 23$ & 0.9500 \\
\hline $\mathrm{C} 10-\mathrm{H} 10$ & 0.9500 & $\mathrm{C} 24-\mathrm{C} 25$ & $1.3871(16)$ \\
\hline $\mathrm{C} 12-\mathrm{C} 13$ & $1.4637(14)$ & $\mathrm{C} 24-\mathrm{H} 24$ & 0.9500 \\
\hline $\mathrm{C} 12-\mathrm{H} 12$ & 0.9500 & $\mathrm{C} 25-\mathrm{H} 25$ & 0.9500 \\
\hline $\mathrm{O} 1-\mathrm{C} 1-\mathrm{C} 2$ & $121.81(9)$ & $\mathrm{C} 13-\mathrm{C} 12-\mathrm{H} 12$ & 114.0 \\
\hline $\mathrm{O} 1-\mathrm{C} 1-\mathrm{C} 11$ & $118.85(9)$ & $\mathrm{C} 14-\mathrm{C} 13-\mathrm{C} 18$ & $116.95(9)$ \\
\hline $\mathrm{C} 2-\mathrm{C} 1-\mathrm{C} 11$ & $119.33(9)$ & $\mathrm{C} 14-\mathrm{C} 13-\mathrm{C} 12$ & $125.98(9)$ \\
\hline $\mathrm{C} 12-\mathrm{C} 2-\mathrm{C} 1$ & $116.43(9)$ & $\mathrm{C} 18-\mathrm{C} 13-\mathrm{C} 12$ & $117.06(9)$ \\
\hline
\end{tabular}




\begin{tabular}{|c|c|c|c|}
\hline $\mathrm{C} 12-\mathrm{C} 2-\mathrm{C} 3$ & $127.54(9)$ & $\mathrm{C} 15-\mathrm{C} 14-\mathrm{C} 13$ & $121.79(10)$ \\
\hline $\mathrm{C} 1-\mathrm{C} 2-\mathrm{C} 3$ & $116.03(9)$ & $\mathrm{C} 15-\mathrm{C} 14-\mathrm{H} 14$ & 119.1 \\
\hline $\mathrm{C} 2-\mathrm{C} 3-\mathrm{C} 4$ & $111.93(9)$ & $\mathrm{C} 13-\mathrm{C} 14-\mathrm{H} 14$ & 119.1 \\
\hline $\mathrm{C} 2-\mathrm{C} 3-\mathrm{H} 3 \mathrm{~A}$ & 109.2 & $\mathrm{C} 14-\mathrm{C} 15-\mathrm{C} 16$ & $119.59(10)$ \\
\hline $\mathrm{C} 4-\mathrm{C} 3-\mathrm{H} 3 \mathrm{~A}$ & 109.2 & $\mathrm{C} 14-\mathrm{C} 15-\mathrm{H} 15$ & 120.2 \\
\hline $\mathrm{C} 2-\mathrm{C} 3-\mathrm{H} 3 \mathrm{~B}$ & 109.2 & $\mathrm{C} 16-\mathrm{C} 15-\mathrm{H} 15$ & 120.2 \\
\hline $\mathrm{C} 4-\mathrm{C} 3-\mathrm{H} 3 \mathrm{~B}$ & 109.2 & $\mathrm{O} 2-\mathrm{C} 16-\mathrm{C} 15$ & $124.70(9)$ \\
\hline $\mathrm{H} 3 \mathrm{~A}-\mathrm{C} 3-\mathrm{H} 3 \mathrm{~B}$ & 107.9 & $\mathrm{O} 2-\mathrm{C} 16-\mathrm{C} 17$ & $115.57(9)$ \\
\hline $\mathrm{C} 3-\mathrm{C} 4-\mathrm{C} 5$ & $112.28(9)$ & $\mathrm{C} 15-\mathrm{C} 16-\mathrm{C} 17$ & $119.73(10)$ \\
\hline $\mathrm{C} 3-\mathrm{C} 4-\mathrm{H} 4 \mathrm{~A}$ & 109.1 & $\mathrm{C} 18-\mathrm{C} 17-\mathrm{C} 16$ & $119.83(10)$ \\
\hline $\mathrm{C} 5-\mathrm{C} 4-\mathrm{H} 4 \mathrm{~A}$ & 109.1 & $\mathrm{C} 18-\mathrm{C} 17-\mathrm{H} 17$ & 120.1 \\
\hline $\mathrm{C} 3-\mathrm{C} 4-\mathrm{H} 4 \mathrm{~B}$ & 109.1 & $\mathrm{C} 16-\mathrm{C} 17-\mathrm{H} 17$ & 120.1 \\
\hline $\mathrm{C} 5-\mathrm{C} 4-\mathrm{H} 4 \mathrm{~B}$ & 109.1 & $\mathrm{C} 17-\mathrm{C} 18-\mathrm{C} 13$ & $122.01(9)$ \\
\hline $\mathrm{H} 4 \mathrm{~A}-\mathrm{C} 4-\mathrm{H} 4 \mathrm{~B}$ & 107.9 & $\mathrm{C} 17-\mathrm{C} 18-\mathrm{H} 18$ & 119.0 \\
\hline $\mathrm{C} 6-\mathrm{C} 5-\mathrm{C} 4$ & $114.06(9)$ & $\mathrm{C} 13-\mathrm{C} 18-\mathrm{H} 18$ & 119.0 \\
\hline $\mathrm{C} 6-\mathrm{C} 5-\mathrm{H} 5 \mathrm{~A}$ & 108.7 & $\mathrm{O} 2-\mathrm{C} 19-\mathrm{C} 20$ & $108.38(8)$ \\
\hline $\mathrm{C} 4-\mathrm{C} 5-\mathrm{H} 5 \mathrm{~A}$ & 108.7 & $\mathrm{O} 2-\mathrm{C} 19-\mathrm{H} 19 \mathrm{~A}$ & 110.0 \\
\hline $\mathrm{C} 6-\mathrm{C} 5-\mathrm{H} 5 \mathrm{~B}$ & 108.7 & $\mathrm{C} 20-\mathrm{C} 19-\mathrm{H} 19 \mathrm{~A}$ & 110.0 \\
\hline $\mathrm{C} 4-\mathrm{C} 5-\mathrm{H} 5 \mathrm{~B}$ & 108.7 & $\mathrm{O} 2-\mathrm{C} 19-\mathrm{H} 19 \mathrm{~B}$ & 110.0 \\
\hline $\mathrm{H} 5 \mathrm{~A}-\mathrm{C} 5-\mathrm{H} 5 \mathrm{~B}$ & 107.6 & $\mathrm{C} 20-\mathrm{C} 19-\mathrm{H} 19 \mathrm{~B}$ & 110.0 \\
\hline $\mathrm{C} 7-\mathrm{C} 6-\mathrm{C} 11$ & $118.30(10)$ & $\mathrm{H} 19 \mathrm{~A}-\mathrm{C} 19-\mathrm{H} 19 \mathrm{~B}$ & 108.4 \\
\hline $\mathrm{C} 7-\mathrm{C} 6-\mathrm{C} 5$ & $120.45(10)$ & $\mathrm{C} 25-\mathrm{C} 20-\mathrm{C} 21$ & $118.80(10)$ \\
\hline $\mathrm{C} 11-\mathrm{C} 6-\mathrm{C} 5$ & $121.17(9)$ & $\mathrm{C} 25-\mathrm{C} 20-\mathrm{C} 19$ & $121.35(10)$ \\
\hline $\mathrm{C} 8-\mathrm{C} 7-\mathrm{C} 6$ & $121.25(11)$ & $\mathrm{C} 21-\mathrm{C} 20-\mathrm{C} 19$ & $119.83(10)$ \\
\hline $\mathrm{C} 8-\mathrm{C} 7-\mathrm{H} 7$ & 119.4 & $\mathrm{C} 22-\mathrm{C} 21-\mathrm{C} 20$ & $120.46(10)$ \\
\hline $\mathrm{C} 6-\mathrm{C} 7-\mathrm{H} 7$ & 119.4 & $\mathrm{C} 22-\mathrm{C} 21-\mathrm{H} 21$ & 119.8 \\
\hline $\mathrm{C} 9-\mathrm{C} 8-\mathrm{C} 7$ & $120.17(10)$ & $\mathrm{C} 20-\mathrm{C} 21-\mathrm{H} 21$ & 119.8 \\
\hline $\mathrm{C} 9-\mathrm{C} 8-\mathrm{H} 8$ & 119.9 & $\mathrm{C} 21-\mathrm{C} 22-\mathrm{C} 23$ & $120.37(10)$ \\
\hline $\mathrm{C} 7-\mathrm{C} 8-\mathrm{H} 8$ & 119.9 & $\mathrm{C} 21-\mathrm{C} 22-\mathrm{H} 22$ & 119.8 \\
\hline $\mathrm{C} 8-\mathrm{C} 9-\mathrm{C} 10$ & $119.62(11)$ & $\mathrm{C} 23-\mathrm{C} 22-\mathrm{H} 22$ & 119.8 \\
\hline $\mathrm{C} 8-\mathrm{C} 9-\mathrm{H} 9$ & 120.2 & $\mathrm{C} 24-\mathrm{C} 23-\mathrm{C} 22$ & $119.60(10)$ \\
\hline $\mathrm{C} 10-\mathrm{C} 9-\mathrm{H} 9$ & 120.2 & $\mathrm{C} 24-\mathrm{C} 23-\mathrm{H} 23$ & 120.2 \\
\hline $\mathrm{C} 9-\mathrm{C} 10-\mathrm{C} 11$ & $120.72(11)$ & $\mathrm{C} 22-\mathrm{C} 23-\mathrm{H} 23$ & 120.2 \\
\hline $\mathrm{C} 9-\mathrm{C} 10-\mathrm{H} 10$ & 119.6 & $\mathrm{C} 23-\mathrm{C} 24-\mathrm{C} 25$ & $120.12(10)$ \\
\hline $\mathrm{C} 11-\mathrm{C} 10-\mathrm{H} 10$ & 119.6 & $\mathrm{C} 23-\mathrm{C} 24-\mathrm{H} 24$ & 119.9 \\
\hline $\mathrm{C} 10-\mathrm{C} 11-\mathrm{C} 6$ & $119.92(10)$ & $\mathrm{C} 25-\mathrm{C} 24-\mathrm{H} 24$ & 119.9 \\
\hline $\mathrm{C} 10-\mathrm{C} 11-\mathrm{C} 1$ & $117.49(9)$ & $\mathrm{C} 24-\mathrm{C} 25-\mathrm{C} 20$ & $120.64(10)$ \\
\hline $\mathrm{C} 6-\mathrm{C} 11-\mathrm{C} 1$ & $122.50(9)$ & $\mathrm{C} 24-\mathrm{C} 25-\mathrm{H} 25$ & 119.7 \\
\hline $\mathrm{C} 2-\mathrm{C} 12-\mathrm{C} 13$ & $132.05(9)$ & $\mathrm{C} 20-\mathrm{C} 25-\mathrm{H} 25$ & 119.7 \\
\hline $\mathrm{C} 2-\mathrm{C} 12-\mathrm{H} 12$ & 114.0 & $\mathrm{C} 16-\mathrm{O} 2-\mathrm{C} 19$ & $116.40(8)$ \\
\hline $\mathrm{O} 1-\mathrm{C} 1-\mathrm{C} 2-\mathrm{C} 12$ & $20.98(15)$ & $\mathrm{C} 3-\mathrm{C} 2-\mathrm{C} 12-\mathrm{C} 13$ & $0.73(19)$ \\
\hline $\mathrm{C} 11-\mathrm{C} 1-\mathrm{C} 2-\mathrm{C} 12$ & $-159.81(9)$ & $\mathrm{C} 2-\mathrm{C} 12-\mathrm{C} 13-\mathrm{C} 14$ & $-17.70(19)$ \\
\hline $\mathrm{O} 1-\mathrm{C} 1-\mathrm{C} 2-\mathrm{C} 3$ & $-159.38(10)$ & $\mathrm{C} 2-\mathrm{C} 12-\mathrm{C} 13-\mathrm{C} 18$ & $163.17(11)$ \\
\hline $\mathrm{C} 11-\mathrm{C} 1-\mathrm{C} 2-\mathrm{C} 3$ & $19.82(13)$ & $\mathrm{C} 18-\mathrm{C} 13-\mathrm{C} 14-\mathrm{C} 15$ & $-2.86(16)$ \\
\hline $\mathrm{C} 12-\mathrm{C} 2-\mathrm{C} 3-\mathrm{C} 4$ & $95.36(13)$ & $\mathrm{C} 12-\mathrm{C} 13-\mathrm{C} 14-\mathrm{C} 15$ & $178.01(10)$ \\
\hline $\mathrm{C} 1-\mathrm{C} 2-\mathrm{C} 3-\mathrm{C} 4$ & $-84.23(11)$ & $\mathrm{C} 13-\mathrm{C} 14-\mathrm{C} 15-\mathrm{C} 16$ & $0.48(16)$ \\
\hline $\mathrm{C} 2-\mathrm{C} 3-\mathrm{C} 4-\mathrm{C} 5$ & $43.11(12)$ & $\mathrm{C} 14-\mathrm{C} 15-\mathrm{C} 16-\mathrm{O} 2$ & $-178.18(10)$ \\
\hline
\end{tabular}




$$
\begin{aligned}
& \mathrm{C} 3-\mathrm{C} 4-\mathrm{C} 5-\mathrm{C} 6 \\
& \mathrm{C} 4-\mathrm{C} 5-\mathrm{C} 6-\mathrm{C} 7 \\
& \mathrm{C} 4-\mathrm{C} 5-\mathrm{C} 6-\mathrm{C} 11 \\
& \mathrm{C} 11-\mathrm{C} 6-\mathrm{C} 7-\mathrm{C} 8 \\
& \mathrm{C} 5-\mathrm{C} 6-\mathrm{C} 7-\mathrm{C} 8 \\
& \mathrm{C} 6-\mathrm{C} 7-\mathrm{C} 8-\mathrm{C} 9 \\
& \mathrm{C} 7-\mathrm{C} 8-\mathrm{C} 9-\mathrm{C} 10 \\
& \mathrm{C} 8-\mathrm{C} 9-\mathrm{C} 10-\mathrm{C} 11 \\
& \mathrm{C} 9-\mathrm{C} 10-\mathrm{C} 11-\mathrm{C} 6 \\
& \mathrm{C} 9-\mathrm{C} 10-\mathrm{C} 11-\mathrm{C} 1 \\
& \mathrm{C} 7-\mathrm{C} 6-\mathrm{C} 11-\mathrm{C} 10 \\
& \mathrm{C} 5-\mathrm{C} 6-\mathrm{C} 11-\mathrm{C} 10 \\
& \mathrm{C} 7-\mathrm{C} 6-\mathrm{C} 11-\mathrm{C} 1 \\
& \mathrm{C} 5-\mathrm{C} 6-\mathrm{C} 11-\mathrm{C} 1 \\
& \mathrm{O} 1-\mathrm{C} 1-\mathrm{C} 11-\mathrm{C} 10 \\
& \mathrm{C} 2-\mathrm{C} 1-\mathrm{C} 11-\mathrm{C} 10 \\
& \mathrm{O} 1-\mathrm{C} 1-\mathrm{C} 11-\mathrm{C} 6 \\
& \mathrm{C} 2-\mathrm{C} 1-\mathrm{C} 11-\mathrm{C} 6 \\
& \mathrm{C} 1-\mathrm{C} 2-\mathrm{C} 12-\mathrm{C} 13
\end{aligned}
$$

$$
\begin{aligned}
& \mathrm{C} 14-\mathrm{C} 15-\mathrm{C} 16-\mathrm{C} 17 \\
& \mathrm{O} 2-\mathrm{C} 16-\mathrm{C} 17-\mathrm{C} 18 \\
& \mathrm{C} 15-\mathrm{C} 16-\mathrm{C} 17-\mathrm{C} 18 \\
& \mathrm{C} 16-\mathrm{C} 17-\mathrm{C} 18-\mathrm{C} 13 \\
& \mathrm{C} 14-\mathrm{C} 13-\mathrm{C} 18-\mathrm{C} 17 \\
& \mathrm{C} 12-\mathrm{C} 13-\mathrm{C} 18-\mathrm{C} 17 \\
& \mathrm{O} 2-\mathrm{C} 19-\mathrm{C} 20-\mathrm{C} 25 \\
& \mathrm{O} 2-\mathrm{C} 19-\mathrm{C} 20-\mathrm{C} 21 \\
& \mathrm{C} 25-\mathrm{C} 20-\mathrm{C} 21-\mathrm{C} 22 \\
& \mathrm{C} 19-\mathrm{C} 20-\mathrm{C} 21-\mathrm{C} 22 \\
& \mathrm{C} 20-\mathrm{C} 21-\mathrm{C} 22-\mathrm{C} 23 \\
& \mathrm{C} 21-\mathrm{C} 22-\mathrm{C} 23-\mathrm{C} 24 \\
& \mathrm{C} 22-\mathrm{C} 23-\mathrm{C} 24-\mathrm{C} 25 \\
& \mathrm{C} 23-\mathrm{C} 24-\mathrm{C} 25-\mathrm{C} 20 \\
& \mathrm{C} 21-\mathrm{C} 20-\mathrm{C} 25-\mathrm{C} 24 \\
& \mathrm{C} 19-\mathrm{C} 20-\mathrm{C} 25-\mathrm{C} 24 \\
& \mathrm{C} 15-\mathrm{C} 16-\mathrm{O} 2-\mathrm{C} 19 \\
& \mathrm{C} 17-\mathrm{C} 16-\mathrm{O} 2-\mathrm{C} 19 \\
& \mathrm{C} 20-\mathrm{C} 19-\mathrm{O} 2-\mathrm{C} 16
\end{aligned}
$$

Hydrogen-bond geometry $\left(\AA,{ }^{\circ}\right)$

\begin{tabular}{lllll}
\hline$D-\mathrm{H} \cdots A$ & $D-\mathrm{H}$ & $\mathrm{H} \cdots A$ & $D \cdots A$ & $D-\mathrm{H} \cdots A$ \\
\hline $\mathrm{C} 15-\mathrm{H} 15 \cdots \mathrm{O} 1^{\mathrm{i}}$ & 0.95 & 2.40 & $3.3477(13)$ & 176 \\
$\mathrm{C} 18-\mathrm{H} 18 \cdots C g 3^{\mathrm{ii}}$ & 0.95 & 2.64 & $3.5147(13)$ & 153 \\
\hline
\end{tabular}

Symmetry codes: (i) $x+1, y, z$; (ii) $x-1, y, z$.

2-(4-Chlorobenzylidene)-6,7,8,9-tetrahydro-5H-benzo[7]annulen-5-one (IV)

\section{Crystal data}

$\mathrm{C}_{18} \mathrm{H}_{15} \mathrm{ClO}$

$M_{r}=282.75$

Monoclinic, $P 2_{1} / n$

$a=10.6273(5) \AA$

$b=11.6191(4) \AA$

$c=12.1114(5) \AA$

$\beta=108.777(4)^{\circ}$

$V=1415.92(11) \AA^{3}$

$Z=4$

\section{Data collection}

XtaLAB AFC11 (RCD3): quarter-chi single CCD diffractometer

Radiation source: Rotating-anode X-ray tube

Mirror monochromator

$\omega$ scans

Absorption correction: multi-scan

(CrysAlis PRO; Rigaku, 2017)

$T_{\min }=0.722, T_{\max }=1.000$
$F(000)=592$

$D_{\mathrm{x}}=1.326 \mathrm{Mg} \mathrm{m}^{-3}$

$\mathrm{Cu} K \alpha$ radiation, $\lambda=1.54184 \AA$

Cell parameters from 4926 reflections

$\theta=4.8-70.0^{\circ}$

$\mu=2.31 \mathrm{~mm}^{-1}$

$T=100 \mathrm{~K}$

Plate, colourless

$0.28 \times 0.20 \times 0.03 \mathrm{~mm}$

11747 measured reflections

2568 independent reflections

2203 reflections with $I>2 \sigma(I)$

$R_{\text {int }}=0.073$

$\theta_{\text {max }}=68.2^{\circ}, \theta_{\min }=4.8^{\circ}$

$h=-12 \rightarrow 11$

$k=-13 \rightarrow 13$

$l=-14 \rightarrow 13$
$1.83(16)$

$178.36(9)$

$-1.65(16)$

$-0.86(16)$

$3.06(15)$

$-177.73(9)$

$-102.31(11)$

$79.43(12)$

$0.41(15)$

$178.72(9)$

$-1.38(16)$

$1.27(16)$

$-0.20(16)$

-0.77 (16)

0.67 (16)

$-177.61(10)$

0.07 (15)

$-179.94(9)$

-179.68 (9) 


\section{Refinement}

Refinement on $F^{2}$

Least-squares matrix: full

$R\left[F^{2}>2 \sigma\left(F^{2}\right)\right]=0.055$

$w R\left(F^{2}\right)=0.165$

$S=1.11$

2568 reflections

181 parameters

0 restraints
Primary atom site location: structure-invariant direct methods

Hydrogen site location: inferred from neighbouring sites

$\mathrm{H}$-atom parameters constrained

$w=1 /\left[\sigma^{2}\left(F_{\mathrm{o}}^{2}\right)+(0.1145 P)^{2}+0.0344 P\right]$ where $P=\left(F_{\mathrm{o}}{ }^{2}+2 F_{\mathrm{c}}{ }^{2}\right) / 3$

$(\Delta / \sigma)_{\max }=0.001$

$\Delta \rho_{\max }=0.32 \mathrm{e} \AA^{-3}$

$\Delta \rho_{\min }=-0.41$ e $\AA^{-3}$

Special details

Geometry. All esds (except the esd in the dihedral angle between two 1.s. planes) are estimated using the full covariance matrix. The cell esds are taken into account individually in the estimation of esds in distances, angles and torsion angles; correlations between esds in cell parameters are only used when they are defined by crystal symmetry. An approximate (isotropic) treatment of cell esds is used for estimating esds involving l.s. planes.

Fractional atomic coordinates and isotropic or equivalent isotropic displacement parameters $\left(\hat{A}^{2}\right)$

\begin{tabular}{|c|c|c|c|c|}
\hline & $x$ & $y$ & $z$ & $U_{\text {iso }} * / U_{\text {eq }}$ \\
\hline $\mathrm{C} 1$ & $0.2086(2)$ & $0.65193(16)$ & $0.58219(17)$ & $0.0292(4)$ \\
\hline $\mathrm{C} 2$ & 0.34239 (19) & $0.67189(16)$ & $0.67264(17)$ & $0.0292(4)$ \\
\hline $\mathrm{C} 3$ & $0.34206(19)$ & $0.73875(16)$ & $0.77902(17)$ & $0.0311(5)$ \\
\hline $\mathrm{H} 3 \mathrm{~A}$ & 0.4244 & 0.7207 & 0.8436 & $0.037^{*}$ \\
\hline H3B & 0.2658 & 0.7131 & 0.8029 & $0.037 *$ \\
\hline $\mathrm{C} 4$ & $0.3330(2)$ & $0.86976(16)$ & $0.76058(18)$ & $0.0333(5)$ \\
\hline $\mathrm{H} 4 \mathrm{~A}$ & 0.4225 & 0.9004 & 0.7685 & $0.040^{*}$ \\
\hline H4B & 0.3028 & 0.9057 & 0.8218 & $0.040^{*}$ \\
\hline $\mathrm{C} 5$ & $0.2366(2)$ & $0.90247(17)$ & $0.64003(18)$ & $0.0323(5)$ \\
\hline H5A & 0.2215 & 0.9866 & 0.6372 & $0.039 *$ \\
\hline H5B & 0.2775 & 0.8829 & 0.5796 & $0.039 *$ \\
\hline C6 & $0.1047(2)$ & $0.84132(17)$ & $0.61283(17)$ & $0.0301(5)$ \\
\hline $\mathrm{C} 7$ & $-0.0085(2)$ & $0.90160(19)$ & 0.61159 (17) & $0.0353(5)$ \\
\hline H7 & -0.0024 & 0.9820 & 0.6268 & $0.042 *$ \\
\hline $\mathrm{C} 8$ & -0.1309 (2) & $0.84680(19)$ & $0.58856(19)$ & $0.0367(5)$ \\
\hline H8 & -0.2071 & 0.8896 & 0.5885 & $0.044 *$ \\
\hline C9 & $-0.1412(2)$ & $0.72966(19)$ & $0.56571(17)$ & $0.0361(5)$ \\
\hline H9 & -0.2242 & 0.6917 & 0.5506 & $0.043^{*}$ \\
\hline $\mathrm{C} 10$ & $-0.0297(2)$ & $0.66851(18)$ & 0.56509 (17) & $0.0330(5)$ \\
\hline H10 & -0.0369 & 0.5884 & 0.5486 & $0.040^{*}$ \\
\hline $\mathrm{C} 11$ & 0.09310 (19) & $0.72291(16)$ & 0.58837 (16) & $0.0294(5)$ \\
\hline $\mathrm{C} 12$ & $0.4476(2)$ & $0.62505(16)$ & 0.65047 (18) & $0.0317(5)$ \\
\hline H12 & 0.4264 & 0.5825 & 0.5798 & $0.038^{*}$ \\
\hline $\mathrm{C} 13$ & $0.5893(2)$ & $0.62893(16)$ & $0.71748(18)$ & $0.0303(5)$ \\
\hline $\mathrm{C} 14$ & $0.6506(2)$ & $0.71561(17)$ & $0.79718(18)$ & $0.0334(5)$ \\
\hline H14 & 0.5983 & 0.7765 & 0.8116 & $0.040^{*}$ \\
\hline C15 & $0.7861(2)$ & $0.71376(17)$ & $0.85517(18)$ & $0.0339(5)$ \\
\hline H15 & 0.8263 & 0.7730 & 0.9090 & $0.041^{*}$ \\
\hline C16 & $0.8629(2)$ & $0.62534(17)$ & $0.83450(19)$ & $0.0341(5)$ \\
\hline
\end{tabular}




$\begin{array}{lllll}\text { C17 } & 0.8062(2) & 0.53886(18) & 0.75589(19) & 0.0391(5) \\ \text { H17 } & 0.8593 & 0.4783 & 0.7421 & 0.047^{*} \\ \text { C18 } & 0.6709(2) & 0.54195(18) & 0.69764(18) & 0.0360(5) \\ \text { H18 } & 0.6321 & 0.4833 & 0.6425 & 0.043^{*} \\ \text { O1 } & 0.19203(14) & 0.57880(12) & 0.50600(12) & 0.0347(4) \\ \text { C11 } & 1.03346(5) & 0.62611(5) & 0.90563(5) & 0.0469(3)\end{array}$

Atomic displacement parameters $\left(\AA^{2}\right)$

\begin{tabular}{lllllll}
\hline & $U^{11}$ & $U^{22}$ & $U^{33}$ & $U^{12}$ & $U^{13}$ & $U^{23}$ \\
\hline C1 & $0.0308(11)$ & $0.0267(9)$ & $0.0297(10)$ & $-0.0017(8)$ & $0.0091(8)$ & $0.0023(8)$ \\
C2 & $0.0292(10)$ & $0.0258(9)$ & $0.0307(10)$ & $-0.0012(7)$ & $0.0068(8)$ & $0.0018(7)$ \\
C3 & $0.0273(10)$ & $0.0325(10)$ & $0.0309(10)$ & $0.0007(8)$ & $0.0056(8)$ & $-0.0006(8)$ \\
C4 & $0.0274(11)$ & $0.0321(11)$ & $0.0371(12)$ & $-0.0021(8)$ & $0.0056(9)$ & $-0.0064(8)$ \\
C5 & $0.0308(11)$ & $0.0277(9)$ & $0.0362(11)$ & $-0.0004(8)$ & $0.0076(9)$ & $-0.0006(8)$ \\
C6 & $0.0289(11)$ & $0.0296(10)$ & $0.0295(10)$ & $-0.0005(8)$ & $0.0063(8)$ & $0.0004(8)$ \\
C7 & $0.0330(11)$ & $0.0362(11)$ & $0.0330(11)$ & $0.0017(9)$ & $0.0056(9)$ & $-0.0004(9)$ \\
C8 & $0.0286(11)$ & $0.0469(12)$ & $0.0328(11)$ & $0.0045(9)$ & $0.0074(9)$ & $-0.0001(9)$ \\
C9 & $0.0289(11)$ & $0.0455(12)$ & $0.0312(11)$ & $-0.0047(9)$ & $0.0059(9)$ & $0.0015(9)$ \\
C10 & $0.0323(11)$ & $0.0344(11)$ & $0.0295(10)$ & $-0.0043(8)$ & $0.0062(8)$ & $0.0008(8)$ \\
C11 & $0.0280(11)$ & $0.0318(10)$ & $0.0263(10)$ & $0.0004(8)$ & $0.0058(8)$ & $0.0017(7)$ \\
C12 & $0.0350(12)$ & $0.0274(10)$ & $0.0303(11)$ & $-0.0015(8)$ & $0.0073(9)$ & $0.0008(7)$ \\
C13 & $0.0306(11)$ & $0.0317(10)$ & $0.0284(10)$ & $0.0020(8)$ & $0.0090(9)$ & $0.0025(8)$ \\
C14 & $0.0296(11)$ & $0.0314(10)$ & $0.0384(11)$ & $0.0005(8)$ & $0.0099(9)$ & $-0.0030(8)$ \\
C15 & $0.0325(11)$ & $0.0338(10)$ & $0.0350(11)$ & $-0.0023(8)$ & $0.0101(9)$ & $-0.0016(8)$ \\
C16 & $0.0281(11)$ & $0.0383(11)$ & $0.0356(12)$ & $0.0005(8)$ & $0.0099(9)$ & $0.0045(8)$ \\
C17 & $0.0339(11)$ & $0.0373(12)$ & $0.0465(13)$ & $0.0049(9)$ & $0.0134(10)$ & $-0.0025(9)$ \\
C18 & $0.0334(11)$ & $0.0344(11)$ & $0.0397(12)$ & $-0.0005(8)$ & $0.0110(9)$ & $-0.0059(8)$ \\
O1 & $0.0348(8)$ & $0.0318(8)$ & $0.0354(8)$ & $-0.0029(6)$ & $0.0083(6)$ & $-0.0060(6)$ \\
C11 & $0.0268(4)$ & $0.0552(4)$ & $0.0545(4)$ & $0.0032(2)$ & $0.0071(3)$ & $0.0000(2)$ \\
& & & & & & \\
\hline
\end{tabular}

Geometric parameters $\left(\AA,{ }^{\circ}\right)$

\begin{tabular}{llll}
\hline $\mathrm{C} 1-\mathrm{O} 1$ & $1.225(2)$ & $\mathrm{C} 8-\mathrm{H} 8$ & 0.9500 \\
$\mathrm{C} 1-\mathrm{C} 11$ & $1.501(3)$ & $\mathrm{C} 9-\mathrm{C} 10$ & $1.384(3)$ \\
$\mathrm{C} 1-\mathrm{C} 2$ & $1.507(3)$ & $\mathrm{C} 9-\mathrm{H} 9$ & 0.9500 \\
$\mathrm{C} 2-\mathrm{C} 12$ & $1.346(3)$ & $\mathrm{C} 10-\mathrm{C} 11$ & $1.395(3)$ \\
$\mathrm{C} 2-\mathrm{C} 3$ & $1.506(3)$ & $\mathrm{C} 10-\mathrm{H} 10$ & 0.9500 \\
$\mathrm{C} 3-\mathrm{C} 4$ & $1.537(3)$ & $\mathrm{C} 12-\mathrm{C} 13$ & $1.464(3)$ \\
$\mathrm{C} 3-\mathrm{H} 3 \mathrm{~A}$ & 0.9900 & $\mathrm{C} 12-\mathrm{H} 12$ & 0.9500 \\
$\mathrm{C} 3-\mathrm{H} 3 \mathrm{~B}$ & 0.9900 & $\mathrm{C} 13-\mathrm{C} 18$ & $1.402(3)$ \\
$\mathrm{C} 4-\mathrm{C} 5$ & $1.537(3)$ & $\mathrm{C} 13-\mathrm{C} 14$ & $1.402(3)$ \\
$\mathrm{C} 4-\mathrm{H} 4 \mathrm{~A}$ & 0.9900 & $\mathrm{C} 14-\mathrm{C} 15$ & $1.385(3)$ \\
$\mathrm{C} 4-\mathrm{H} 4 \mathrm{~B}$ & 0.9900 & $\mathrm{C} 14-\mathrm{H} 14$ & 0.9500 \\
$\mathrm{C} 5-\mathrm{C} 6$ & $1.510(3)$ & $\mathrm{C} 15-\mathrm{C} 16$ & $1.384(3)$ \\
$\mathrm{C} 5-\mathrm{H} 5 \mathrm{~A}$ & 0.9900 & $\mathrm{C} 15-\mathrm{H} 15$ & 0.9500 \\
$\mathrm{C} 5-\mathrm{H} 5 \mathrm{~B}$ & 0.9900 & $\mathrm{C} 16-\mathrm{C} 17$ & $1.382(3)$ \\
$\mathrm{C} 6-\mathrm{C} 7$ & $1.388(3)$ & $\mathrm{C} 16-\mathrm{C} 11$ & $1.739(2)$
\end{tabular}




\begin{tabular}{|c|c|c|c|}
\hline $\mathrm{C} 6-\mathrm{C} 11$ & $1.404(3)$ & $\mathrm{C} 17-\mathrm{C} 18$ & $1.383(3)$ \\
\hline $\mathrm{C} 7-\mathrm{C} 8$ & $1.393(3)$ & $\mathrm{C} 17-\mathrm{H} 17$ & 0.9500 \\
\hline $\mathrm{C} 7-\mathrm{H} 7$ & 0.9500 & $\mathrm{C} 18-\mathrm{H} 18$ & 0.9500 \\
\hline $\mathrm{C} 8-\mathrm{C} 9$ & $1.386(3)$ & & \\
\hline $\mathrm{O} 1-\mathrm{C} 1-\mathrm{C} 11$ & $119.82(18)$ & $\mathrm{C} 7-\mathrm{C} 8-\mathrm{H} 8$ & 120.1 \\
\hline $\mathrm{O} 1-\mathrm{C} 1-\mathrm{C} 2$ & $121.84(18)$ & $\mathrm{C} 10-\mathrm{C} 9-\mathrm{C} 8$ & $119.48(19)$ \\
\hline $\mathrm{C} 11-\mathrm{C} 1-\mathrm{C} 2$ & $118.33(16)$ & $\mathrm{C} 10-\mathrm{C} 9-\mathrm{H} 9$ & 120.3 \\
\hline $\mathrm{C} 12-\mathrm{C} 2-\mathrm{C} 3$ & $127.61(18)$ & $\mathrm{C} 8-\mathrm{C} 9-\mathrm{H} 9$ & 120.3 \\
\hline $\mathrm{C} 12-\mathrm{C} 2-\mathrm{C} 1$ & $116.26(17)$ & $\mathrm{C} 9-\mathrm{C} 10-\mathrm{C} 11$ & $120.90(19)$ \\
\hline $\mathrm{C} 3-\mathrm{C} 2-\mathrm{C} 1$ & $116.09(16)$ & $\mathrm{C} 9-\mathrm{C} 10-\mathrm{H} 10$ & 119.6 \\
\hline $\mathrm{C} 2-\mathrm{C} 3-\mathrm{C} 4$ & $113.82(16)$ & $\mathrm{C} 11-\mathrm{C} 10-\mathrm{H} 10$ & 119.6 \\
\hline $\mathrm{C} 2-\mathrm{C} 3-\mathrm{H} 3 \mathrm{~A}$ & 108.8 & $\mathrm{C} 10-\mathrm{C} 11-\mathrm{C} 6$ & $119.99(18)$ \\
\hline $\mathrm{C} 4-\mathrm{C} 3-\mathrm{H} 3 \mathrm{~A}$ & 108.8 & $\mathrm{C} 10-\mathrm{C} 11-\mathrm{C} 1$ & $117.91(17)$ \\
\hline $\mathrm{C} 2-\mathrm{C} 3-\mathrm{H} 3 \mathrm{~B}$ & 108.8 & $\mathrm{C} 6-\mathrm{C} 11-\mathrm{C} 1$ & $122.07(17)$ \\
\hline $\mathrm{C} 4-\mathrm{C} 3-\mathrm{H} 3 \mathrm{~B}$ & 108.8 & $\mathrm{C} 2-\mathrm{C} 12-\mathrm{C} 13$ & $130.51(18)$ \\
\hline $\mathrm{H} 3 \mathrm{~A}-\mathrm{C} 3-\mathrm{H} 3 \mathrm{~B}$ & 107.7 & $\mathrm{C} 2-\mathrm{C} 12-\mathrm{H} 12$ & 114.7 \\
\hline $\mathrm{C} 3-\mathrm{C} 4-\mathrm{C} 5$ & $111.98(16)$ & $\mathrm{C} 13-\mathrm{C} 12-\mathrm{H} 12$ & 114.7 \\
\hline $\mathrm{C} 3-\mathrm{C} 4-\mathrm{H} 4 \mathrm{~A}$ & 109.2 & $\mathrm{C} 18-\mathrm{C} 13-\mathrm{C} 14$ & $117.39(19)$ \\
\hline $\mathrm{C} 5-\mathrm{C} 4-\mathrm{H} 4 \mathrm{~A}$ & 109.2 & $\mathrm{C} 18-\mathrm{C} 13-\mathrm{C} 12$ & $117.75(18)$ \\
\hline $\mathrm{C} 3-\mathrm{C} 4-\mathrm{H} 4 \mathrm{~B}$ & 109.2 & $\mathrm{C} 14-\mathrm{C} 13-\mathrm{C} 12$ & $124.81(17)$ \\
\hline $\mathrm{C} 5-\mathrm{C} 4-\mathrm{H} 4 \mathrm{~B}$ & 109.2 & $\mathrm{C} 15-\mathrm{C} 14-\mathrm{C} 13$ & $120.99(18)$ \\
\hline $\mathrm{H} 4 \mathrm{~A}-\mathrm{C} 4-\mathrm{H} 4 \mathrm{~B}$ & 107.9 & $\mathrm{C} 15-\mathrm{C} 14-\mathrm{H} 14$ & 119.5 \\
\hline $\mathrm{C} 6-\mathrm{C} 5-\mathrm{C} 4$ & $112.27(17)$ & $\mathrm{C} 13-\mathrm{C} 14-\mathrm{H} 14$ & 119.5 \\
\hline $\mathrm{C} 6-\mathrm{C} 5-\mathrm{H} 5 \mathrm{~A}$ & 109.1 & $\mathrm{C} 16-\mathrm{C} 15-\mathrm{C} 14$ & $119.82(19)$ \\
\hline $\mathrm{C} 4-\mathrm{C} 5-\mathrm{H} 5 \mathrm{~A}$ & 109.1 & $\mathrm{C} 16-\mathrm{C} 15-\mathrm{H} 15$ & 120.1 \\
\hline $\mathrm{C} 6-\mathrm{C} 5-\mathrm{H} 5 \mathrm{~B}$ & 109.1 & $\mathrm{C} 14-\mathrm{C} 15-\mathrm{H} 15$ & 120.1 \\
\hline $\mathrm{C} 4-\mathrm{C} 5-\mathrm{H} 5 \mathrm{~B}$ & 109.1 & $\mathrm{C} 17-\mathrm{C} 16-\mathrm{C} 15$ & $120.9(2)$ \\
\hline $\mathrm{H} 5 \mathrm{~A}-\mathrm{C} 5-\mathrm{H} 5 \mathrm{~B}$ & 107.9 & $\mathrm{C} 17-\mathrm{C} 16-\mathrm{Cl1}$ & $119.91(16)$ \\
\hline $\mathrm{C} 7-\mathrm{C} 6-\mathrm{C} 11$ & $118.35(19)$ & $\mathrm{C} 15-\mathrm{C} 16-\mathrm{Cl} 1$ & $119.19(17)$ \\
\hline $\mathrm{C} 7-\mathrm{C} 6-\mathrm{C} 5$ & $120.36(18)$ & $\mathrm{C} 16-\mathrm{C} 17-\mathrm{C} 18$ & $118.90(19)$ \\
\hline $\mathrm{C} 11-\mathrm{C} 6-\mathrm{C} 5$ & $121.30(18)$ & $\mathrm{C} 16-\mathrm{C} 17-\mathrm{H} 17$ & 120.6 \\
\hline $\mathrm{C} 6-\mathrm{C} 7-\mathrm{C} 8$ & $121.4(2)$ & $\mathrm{C} 18-\mathrm{C} 17-\mathrm{H} 17$ & 120.6 \\
\hline $\mathrm{C} 6-\mathrm{C} 7-\mathrm{H} 7$ & 119.3 & $\mathrm{C} 17-\mathrm{C} 18-\mathrm{C} 13$ & $122.0(2)$ \\
\hline $\mathrm{C} 8-\mathrm{C} 7-\mathrm{H} 7$ & 119.3 & $\mathrm{C} 17-\mathrm{C} 18-\mathrm{H} 18$ & 119.0 \\
\hline $\mathrm{C} 9-\mathrm{C} 8-\mathrm{C} 7$ & $119.8(2)$ & $\mathrm{C} 13-\mathrm{C} 18-\mathrm{H} 18$ & 119.0 \\
\hline $\mathrm{C} 9-\mathrm{C} 8-\mathrm{H} 8$ & 120.1 & & \\
\hline $\mathrm{O} 1-\mathrm{C} 1-\mathrm{C} 2-\mathrm{C} 12$ & $14.6(3)$ & $\mathrm{C} 5-\mathrm{C} 6-\mathrm{C} 11-\mathrm{C} 1$ & $-3.0(3)$ \\
\hline $\mathrm{C} 11-\mathrm{C} 1-\mathrm{C} 2-\mathrm{C} 12$ & $-166.52(17)$ & $\mathrm{O} 1-\mathrm{C} 1-\mathrm{C} 11-\mathrm{C} 10$ & $38.8(3)$ \\
\hline $\mathrm{O} 1-\mathrm{C} 1-\mathrm{C} 2-\mathrm{C} 3$ & $-163.47(18)$ & $\mathrm{C} 2-\mathrm{C} 1-\mathrm{C} 11-\mathrm{C} 10$ & $-140.10(18)$ \\
\hline $\mathrm{C} 11-\mathrm{C} 1-\mathrm{C} 2-\mathrm{C} 3$ & $15.4(2)$ & $\mathrm{O} 1-\mathrm{C} 1-\mathrm{C} 11-\mathrm{C} 6$ & $-139.2(2)$ \\
\hline $\mathrm{C} 12-\mathrm{C} 2-\mathrm{C} 3-\mathrm{C} 4$ & $101.3(2)$ & $\mathrm{C} 2-\mathrm{C} 1-\mathrm{C} 11-\mathrm{C} 6$ & $42.0(3)$ \\
\hline $\mathrm{C} 1-\mathrm{C} 2-\mathrm{C} 3-\mathrm{C} 4$ & $-80.8(2)$ & $\mathrm{C} 3-\mathrm{C} 2-\mathrm{C} 12-\mathrm{C} 13$ & $-3.2(3)$ \\
\hline $\mathrm{C} 2-\mathrm{C} 3-\mathrm{C} 4-\mathrm{C} 5$ & $39.8(2)$ & $\mathrm{C} 1-\mathrm{C} 2-\mathrm{C} 12-\mathrm{C} 13$ & $178.97(18)$ \\
\hline $\mathrm{C} 3-\mathrm{C} 4-\mathrm{C} 5-\mathrm{C} 6$ & $48.7(2)$ & $\mathrm{C} 2-\mathrm{C} 12-\mathrm{C} 13-\mathrm{C} 18$ & $157.7(2)$ \\
\hline $\mathrm{C} 4-\mathrm{C} 5-\mathrm{C} 6-\mathrm{C} 7$ & $108.5(2)$ & $\mathrm{C} 2-\mathrm{C} 12-\mathrm{C} 13-\mathrm{C} 14$ & $-24.7(3)$ \\
\hline $\mathrm{C} 4-\mathrm{C} 5-\mathrm{C} 6-\mathrm{C} 11$ & $-71.4(2)$ & $\mathrm{C} 18-\mathrm{C} 13-\mathrm{C} 14-\mathrm{C} 15$ & $-1.1(3)$ \\
\hline
\end{tabular}




$\begin{array}{llll}\mathrm{C} 11-\mathrm{C} 6-\mathrm{C} 7-\mathrm{C} 8 & 1.0(3) & \mathrm{C} 12-\mathrm{C} 13-\mathrm{C} 14-\mathrm{C} 15 & -178.65(19) \\ \mathrm{C} 5-\mathrm{C} 6-\mathrm{C} 7-\mathrm{C} 8 & -178.97(19) & \mathrm{C} 13-\mathrm{C} 14-\mathrm{C} 15-\mathrm{C} 16 & 0.0(3) \\ \mathrm{C} 6-\mathrm{C} 7-\mathrm{C} 8-\mathrm{C} 9 & -0.3(3) & \mathrm{C} 14-\mathrm{C} 15-\mathrm{C} 16-\mathrm{C} 17 & 0.5(3) \\ \mathrm{C} 7-\mathrm{C} 8-\mathrm{C} 9-\mathrm{C} 10 & -0.5(3) & \mathrm{C} 14-\mathrm{C} 15-\mathrm{C} 16-\mathrm{C} 11 & 178.57(16) \\ \mathrm{C} 8-\mathrm{C} 9-\mathrm{C} 10-\mathrm{C} 11 & 0.7(3) & \mathrm{C} 15-\mathrm{C} 16-\mathrm{C} 17-\mathrm{C} 18 & 0.1(3) \\ \mathrm{C} 9-\mathrm{C} 10-\mathrm{C} 11-\mathrm{C} 6 & 0.0(3) & \mathrm{C} 11-\mathrm{C} 16-\mathrm{C} 17-\mathrm{C} 18 & -178.02(16) \\ \mathrm{C} 9-\mathrm{C} 10-\mathrm{C} 11-\mathrm{C} 1 & -177.98(18) & \mathrm{C} 16-\mathrm{C} 17-\mathrm{C} 18-\mathrm{C} 13 & -1.2(3) \\ \mathrm{C} 7-\mathrm{C} 6-\mathrm{C} 11-\mathrm{C} 10 & -0.8(3) & \mathrm{C} 14-\mathrm{C} 13-\mathrm{C} 18-\mathrm{C} 17 & 1.6(3) \\ \mathrm{C} 5-\mathrm{C} 6-\mathrm{C} 11-\mathrm{C} 10 & 179.14(17) & \mathrm{C} 12-\mathrm{C} 13-\mathrm{C} 18-\mathrm{C} 17 & 179.39(18) \\ \mathrm{C} 7-\mathrm{C} 6-\mathrm{C} 11-\mathrm{C} 1 & 177.07(17) & & \end{array}$

Hydrogen-bond geometry $\left(\AA,{ }^{\circ}\right)$

\begin{tabular}{lllll}
\hline$D-\mathrm{H} \cdots A$ & $D-\mathrm{H}$ & $\mathrm{H} \cdots A$ & $D \cdots A$ & $D-\mathrm{H} \cdots A$ \\
\hline $\mathrm{C} 10-\mathrm{H} 10 \cdots \mathrm{O} 1^{\mathrm{i}}$ & 0.95 & 2.50 & $3.319(2)$ & 145 \\
$\mathrm{C} 3-\mathrm{H} 3 A \cdots C g 1^{\mathrm{ii}}$ & 0.99 & 2.83 & $3.572(2)$ & 132 \\
\hline
\end{tabular}

Symmetry codes: (i) $-x,-y+1,-z+1$; (ii) $x-1 / 2,-y+3 / 2, z+1 / 2$.

6-(4-Cyanobenzylidene)-6,7,8,9-tetrahydro-5H-benzo[7]annulen-5-one (V)

\section{Crystal data}

\section{$\mathrm{C}_{19} \mathrm{H}_{15} \mathrm{NO}$}

$M_{r}=273.32$

Monoclinic, $P 2_{1} / c$

$a=12.4725(4) \AA$

$b=7.1718(2) \AA$

$c=15.9983(5) \AA$

$\beta=106.120(3)^{\circ}$

$V=1374.79(8) \AA^{3}$

$Z=4$

\section{Data collection}

XtaLAB AFC11 (RCD3): quarter-chi single CCD diffractometer

Radiation source: Rotating-anode X-ray tube Mirror monochromator

$\omega$ scans

Absorption correction: gaussian

(CrysAlis PRO; Rigaku, 2017)

$T_{\min }=0.895, T_{\max }=1.000$

\section{Refinement}

Refinement on $F^{2}$

Least-squares matrix: full

$R\left[F^{2}>2 \sigma\left(F^{2}\right)\right]=0.068$

$w R\left(F^{2}\right)=0.181$

$S=1.06$

2511 reflections

190 parameters

0 restraints
$F(000)=576$

$D_{\mathrm{x}}=1.321 \mathrm{Mg} \mathrm{m}^{-3}$

$\mathrm{Cu} K \alpha$ radiation, $\lambda=1.54184 \AA$

Cell parameters from 3885 reflections

$\theta=5.7-69.4^{\circ}$

$\mu=0.64 \mathrm{~mm}^{-1}$

$T=100 \mathrm{~K}$

Block, colourless

$0.17 \times 0.10 \times 0.03 \mathrm{~mm}$

9732 measured reflections

2511 independent reflections

2302 reflections with $I>2 \sigma(I)$

$R_{\text {int }}=0.059$

$\theta_{\text {max }}=68.2^{\circ}, \theta_{\text {min }}=3.7^{\circ}$

$h=-14 \longrightarrow 15$

$k=-7 \rightarrow 8$

$l=-19 \rightarrow 19$

Primary atom site location: structure-invariant direct methods

Hydrogen site location: inferred from neighbouring sites

$\mathrm{H}$-atom parameters constrained

$w=1 /\left[\sigma^{2}\left(F_{\mathrm{o}}^{2}\right)+(0.1396 P)^{2}+0.1712 P\right]$

where $P=\left(F_{\mathrm{o}}^{2}+2 F_{\mathrm{c}}^{2}\right) / 3$

$(\Delta / \sigma)_{\max }<0.001$

$\Delta \rho_{\max }=0.49$ e $\AA^{-3}$

$\Delta \rho_{\min }=-0.32$ e $\AA^{-3}$ 


\section{Special details}

Geometry. All esds (except the esd in the dihedral angle between two 1.s. planes) are estimated using the full covariance matrix. The cell esds are taken into account individually in the estimation of esds in distances, angles and torsion angles; correlations between esds in cell parameters are only used when they are defined by crystal symmetry. An approximate (isotropic) treatment of cell esds is used for estimating esds involving l.s. planes.

Fractional atomic coordinates and isotropic or equivalent isotropic displacement parameters $\left(\AA^{2}\right)$

\begin{tabular}{|c|c|c|c|c|}
\hline & $x$ & $y$ & $z$ & $U_{\text {iso }} * / U_{\text {eq }}$ \\
\hline $\mathrm{C} 1$ & $0.34825(12)$ & $0.5911(2)$ & $0.45058(9)$ & $0.0284(4)$ \\
\hline $\mathrm{C} 2$ & $0.29525(12)$ & $0.4044(2)$ & $0.42080(9)$ & $0.0275(4)$ \\
\hline $\mathrm{C} 3$ & $0.32674(12)$ & $0.3153(2)$ & $0.34549(9)$ & $0.0279(4)$ \\
\hline $\mathrm{H} 3 \mathrm{~A}$ & 0.3150 & 0.1790 & 0.3474 & $0.033 *$ \\
\hline H3B & 0.4073 & 0.3366 & 0.3529 & $0.033^{*}$ \\
\hline $\mathrm{C} 4$ & $0.26091(13)$ & $0.3882(2)$ & $0.25527(10)$ & $0.0292(4)$ \\
\hline $\mathrm{H} 4 \mathrm{~A}$ & 0.3080 & 0.3783 & 0.2148 & $0.035^{*}$ \\
\hline $\mathrm{H} 4 \mathrm{~B}$ & 0.1941 & 0.3093 & 0.2323 & $0.035^{*}$ \\
\hline C5 & $0.22444(13)$ & $0.5911(2)$ & $0.25887(10)$ & $0.0296(4)$ \\
\hline $\mathrm{H} 5 \mathrm{~A}$ & 0.1850 & 0.6337 & 0.1994 & $0.036^{*}$ \\
\hline H5B & 0.1717 & 0.5993 & 0.2950 & $0.036^{*}$ \\
\hline C6 & $0.32277(12)$ & $0.7168(2)$ & $0.29642(9)$ & $0.0279(4)$ \\
\hline $\mathrm{C} 7$ & $0.35894(13)$ & $0.8371(2)$ & $0.24193(10)$ & $0.0304(4)$ \\
\hline H7 & 0.3192 & 0.8421 & 0.1820 & $0.037 *$ \\
\hline $\mathrm{C} 8$ & $0.45193(14)$ & $0.9506(2)$ & $0.27303(11)$ & $0.0319(4)$ \\
\hline H8 & 0.4759 & 1.0306 & 0.2344 & $0.038^{*}$ \\
\hline $\mathrm{C} 9$ & $0.50973(13)$ & $0.9462(2)$ & 0.36109 (11) & $0.0318(4)$ \\
\hline H9 & 0.5734 & 1.0232 & 0.3829 & $0.038^{*}$ \\
\hline $\mathrm{C} 10$ & $0.47389(13)$ & $0.8293(2)$ & $0.41635(10)$ & $0.0305(4)$ \\
\hline H10 & 0.5128 & 0.8279 & 0.4765 & $0.037 *$ \\
\hline C11 & $0.38136(12)$ & $0.7129(2)$ & $0.38553(10)$ & $0.0279(4)$ \\
\hline $\mathrm{C} 12$ & $0.23202(13)$ & $0.3273(2)$ & $0.46664(10)$ & $0.0294(4)$ \\
\hline H12 & 0.2248 & 0.3944 & 0.5160 & $0.035^{*}$ \\
\hline $\mathrm{C} 13$ & $0.17236(13)$ & 0.1477 (2) & $0.44785(10)$ & $0.0284(4)$ \\
\hline $\mathrm{C} 14$ & $0.11644(13)$ & 0.0939 (2) & $0.36261(10)$ & $0.0304(4)$ \\
\hline H14 & 0.1162 & 0.1752 & 0.3157 & $0.036^{*}$ \\
\hline $\mathrm{C} 15$ & $0.06168(13)$ & $-0.0752(2)$ & $0.34565(10)$ & $0.0304(4)$ \\
\hline H15 & 0.0252 & -0.1102 & 0.2874 & $0.036^{*}$ \\
\hline $\mathrm{C} 16$ & $0.05996(12)$ & $-0.1944(2)$ & $0.41403(10)$ & $0.0288(4)$ \\
\hline $\mathrm{C} 17$ & $0.11421(14)$ & $-0.1427(2)$ & $0.49975(10)$ & $0.0327(4)$ \\
\hline H17 & 0.1138 & -0.2237 & 0.5466 & $0.039 *$ \\
\hline $\mathrm{C} 18$ & $0.16847(13)$ & $0.0273(2)$ & $0.51552(10)$ & $0.0320(4)$ \\
\hline H18 & 0.2040 & 0.0630 & 0.5738 & $0.038^{*}$ \\
\hline C19 & -0.00015 (13) & $-0.3676(2)$ & $0.39548(10)$ & $0.0308(4)$ \\
\hline N1 & $-0.05047(12)$ & $-0.5036(2)$ & $0.37711(9)$ & $0.0374(4)$ \\
\hline $\mathrm{O} 1$ & $0.36770(11)$ & $0.63971(17)$ & $0.52639(7)$ & $0.0376(4)$ \\
\hline
\end{tabular}


Atomic displacement parameters $\left(\AA^{2}\right)$

\begin{tabular}{lllllll}
\hline & $U^{11}$ & $U^{22}$ & $U^{\beta 3}$ & $U^{12}$ & $U^{13}$ & $U^{23}$ \\
\hline C1 & $0.0239(8)$ & $0.0341(9)$ & $0.0233(8)$ & $0.0023(6)$ & $0.0002(6)$ & $-0.0017(6)$ \\
C2 & $0.0235(7)$ & $0.0299(8)$ & $0.0234(8)$ & $0.0018(6)$ & $-0.0028(6)$ & $0.0020(6)$ \\
C3 & $0.0228(7)$ & $0.0306(8)$ & $0.0273(8)$ & $0.0001(6)$ & $0.0020(6)$ & $-0.0004(6)$ \\
C4 & $0.0263(8)$ & $0.0345(9)$ & $0.0243(8)$ & $-0.0036(6)$ & $0.0030(6)$ & $-0.0032(6)$ \\
C5 & $0.0256(8)$ & $0.0356(9)$ & $0.0231(8)$ & $-0.0009(6)$ & $-0.0009(6)$ & $0.0006(6)$ \\
C6 & $0.0248(8)$ & $0.0304(8)$ & $0.0264(8)$ & $0.0042(6)$ & $0.0034(6)$ & $-0.0011(6)$ \\
C7 & $0.0278(8)$ & $0.0311(8)$ & $0.0307(9)$ & $0.0048(6)$ & $0.0054(7)$ & $0.0001(6)$ \\
C8 & $0.0314(8)$ & $0.0286(8)$ & $0.0375(9)$ & $0.0031(6)$ & $0.0122(7)$ & $0.0008(6)$ \\
C9 & $0.0244(8)$ & $0.0305(8)$ & $0.0391(9)$ & $-0.0001(6)$ & $0.0065(7)$ & $-0.0044(7)$ \\
C10 & $0.0254(8)$ & $0.0307(8)$ & $0.0317(9)$ & $0.0019(6)$ & $0.0016(6)$ & $-0.0038(6)$ \\
C11 & $0.0235(7)$ & $0.0288(8)$ & $0.0292(8)$ & $0.0015(6)$ & $0.0035(6)$ & $-0.0021(6)$ \\
C12 & $0.0272(8)$ & $0.0323(8)$ & $0.0243(8)$ & $0.0018(6)$ & $-0.0002(6)$ & $-0.0001(6)$ \\
C13 & $0.0236(8)$ & $0.0321(9)$ & $0.0277(8)$ & $0.0015(6)$ & $0.0042(6)$ & $-0.0011(6)$ \\
C14 & $0.0274(8)$ & $0.0340(9)$ & $0.0257(8)$ & $-0.0011(6)$ & $0.0009(6)$ & $0.0060(6)$ \\
C15 & $0.0263(8)$ & $0.0354(9)$ & $0.0248(8)$ & $-0.0013(6)$ & $-0.0006(6)$ & $0.0005(6)$ \\
C16 & $0.0239(7)$ & $0.0300(8)$ & $0.0294(8)$ & $0.0004(6)$ & $0.0025(6)$ & $0.0006(6)$ \\
C17 & $0.0332(8)$ & $0.0359(9)$ & $0.0256(8)$ & $-0.0014(7)$ & $0.0027(7)$ & $0.0045(6)$ \\
C18 & $0.0312(8)$ & $0.0371(9)$ & $0.0245(8)$ & $-0.0022(7)$ & $0.0024(6)$ & $-0.0005(6)$ \\
C19 & $0.0300(8)$ & $0.0339(9)$ & $0.0259(8)$ & $0.0023(7)$ & $0.0033(6)$ & $0.0036(6)$ \\
N1 & $0.0377(8)$ & $0.0359(9)$ & $0.0338(8)$ & $-0.0053(6)$ & $0.0018(6)$ & $0.0021(6)$ \\
O1 & $0.0426(7)$ & $0.0412(7)$ & $0.0259(6)$ & $-0.0094(5)$ & $0.0042(5)$ & $-0.0047(5)$ \\
& & & & & & \\
\hline & & & & & &
\end{tabular}

Geometric parameters $\left(\AA,{ }^{\circ}\right)$

\begin{tabular}{llll}
\hline $\mathrm{C} 1-\mathrm{O} 1$ & $1.2199(19)$ & $\mathrm{C} 8-\mathrm{H} 8$ & 0.9500 \\
$\mathrm{C} 1-\mathrm{C} 11$ & $1.502(2)$ & $\mathrm{C} 9-\mathrm{C} 10$ & $1.380(2)$ \\
$\mathrm{C} 1-\mathrm{C} 2$ & $1.510(2)$ & $\mathrm{C} 9-\mathrm{H} 9$ & 0.9500 \\
$\mathrm{C} 2-\mathrm{C} 12$ & $1.337(2)$ & $\mathrm{C} 10-\mathrm{C} 11$ & $1.399(2)$ \\
$\mathrm{C} 2-\mathrm{C} 3$ & $1.509(2)$ & $\mathrm{C} 10-\mathrm{H} 10$ & 0.9500 \\
$\mathrm{C} 3-\mathrm{C} 4$ & $1.540(2)$ & $\mathrm{C} 12-\mathrm{C} 13$ & $1.476(2)$ \\
$\mathrm{C} 3-\mathrm{H} 3 \mathrm{~A}$ & 0.9900 & $\mathrm{C} 12-\mathrm{H} 12$ & 0.9500 \\
$\mathrm{C} 3-\mathrm{H} 3 \mathrm{~B}$ & 0.9900 & $\mathrm{C} 13-\mathrm{C} 18$ & $1.396(2)$ \\
$\mathrm{C} 4-\mathrm{C} 5$ & $1.531(2)$ & $\mathrm{C} 13-\mathrm{C} 14$ & $1.402(2)$ \\
$\mathrm{C} 4-\mathrm{H} 4 \mathrm{~A}$ & 0.9900 & $\mathrm{C} 14-\mathrm{C} 15$ & $1.381(2)$ \\
$\mathrm{C} 4-\mathrm{H} 4 \mathrm{~B}$ & 0.9900 & $\mathrm{C} 14-\mathrm{H} 14$ & 0.9500 \\
$\mathrm{C} 5-\mathrm{C} 6$ & $1.506(2)$ & $\mathrm{C} 15-\mathrm{C} 16$ & $1.393(2)$ \\
$\mathrm{C} 5-\mathrm{H} 5 \mathrm{~A}$ & 0.9900 & $\mathrm{C} 15-\mathrm{H} 15$ & 0.9500 \\
$\mathrm{C} 5-\mathrm{H} 5 \mathrm{~B}$ & 0.9900 & $\mathrm{C} 16-\mathrm{C} 17$ & $1.400(2)$ \\
$\mathrm{C} 6-\mathrm{C} 7$ & $1.388(2)$ & $\mathrm{C} 16-\mathrm{C} 19$ & $1.439(2)$ \\
$\mathrm{C} 6-\mathrm{C} 11$ & $1.410(2)$ & $\mathrm{C} 17-\mathrm{C} 18$ & 0.9500 \\
$\mathrm{C} 7-\mathrm{C} 8$ & $1.391(2)$ & $\mathrm{C} 17-\mathrm{H} 17$ & 0.9500 \\
$\mathrm{C} 7-\mathrm{H} 7$ & 0.9500 & $\mathrm{C} 18-\mathrm{H} 18$ & $1.153(2)$ \\
$\mathrm{C} 8-\mathrm{C} 9$ & $1.393(2)$ & $\mathrm{C} 19-\mathrm{N} 1$ & 120.2 \\
$\mathrm{O} 1-\mathrm{C} 1-\mathrm{C} 11$ & & & $\mathrm{C} 9-\mathrm{C} 8-\mathrm{H} 8$ \\
\end{tabular}




\begin{tabular}{|c|c|c|c|}
\hline $\mathrm{O} 1-\mathrm{C} 1-\mathrm{C} 2$ & $121.05(14)$ & $\mathrm{C} 10-\mathrm{C} 9-\mathrm{C} 8$ & $119.53(15)$ \\
\hline $\mathrm{C} 11-\mathrm{C} 1-\mathrm{C} 2$ & $118.52(13)$ & $\mathrm{C} 10-\mathrm{C} 9-\mathrm{H} 9$ & 120.2 \\
\hline $\mathrm{C} 12-\mathrm{C} 2-\mathrm{C} 3$ & $125.92(15)$ & $\mathrm{C} 8-\mathrm{C} 9-\mathrm{H} 9$ & 120.2 \\
\hline $\mathrm{C} 12-\mathrm{C} 2-\mathrm{C} 1$ & $117.85(14)$ & $\mathrm{C} 9-\mathrm{C} 10-\mathrm{C} 11$ & $121.29(14)$ \\
\hline $\mathrm{C} 3-\mathrm{C} 2-\mathrm{C} 1$ & $116.04(13)$ & $\mathrm{C} 9-\mathrm{C} 10-\mathrm{H} 10$ & 119.4 \\
\hline $\mathrm{C} 2-\mathrm{C} 3-\mathrm{C} 4$ & $114.54(13)$ & $\mathrm{C} 11-\mathrm{C} 10-\mathrm{H} 10$ & 119.4 \\
\hline $\mathrm{C} 2-\mathrm{C} 3-\mathrm{H} 3 \mathrm{~A}$ & 108.6 & $\mathrm{C} 10-\mathrm{C} 11-\mathrm{C} 6$ & $119.32(14)$ \\
\hline $\mathrm{C} 4-\mathrm{C} 3-\mathrm{H} 3 \mathrm{~A}$ & 108.6 & $\mathrm{C} 10-\mathrm{C} 11-\mathrm{C} 1$ & $117.50(14)$ \\
\hline $\mathrm{C} 2-\mathrm{C} 3-\mathrm{H} 3 \mathrm{~B}$ & 108.6 & $\mathrm{C} 6-\mathrm{C} 11-\mathrm{C} 1$ & $123.17(14)$ \\
\hline $\mathrm{C} 4-\mathrm{C} 3-\mathrm{H} 3 \mathrm{~B}$ & 108.6 & $\mathrm{C} 2-\mathrm{C} 12-\mathrm{C} 13$ & $126.19(14)$ \\
\hline $\mathrm{H} 3 \mathrm{~A}-\mathrm{C} 3-\mathrm{H} 3 \mathrm{~B}$ & 107.6 & $\mathrm{C} 2-\mathrm{C} 12-\mathrm{H} 12$ & 116.9 \\
\hline $\mathrm{C} 5-\mathrm{C} 4-\mathrm{C} 3$ & $111.96(12)$ & $\mathrm{C} 13-\mathrm{C} 12-\mathrm{H} 12$ & 116.9 \\
\hline $\mathrm{C} 5-\mathrm{C} 4-\mathrm{H} 4 \mathrm{~A}$ & 109.2 & $\mathrm{C} 18-\mathrm{C} 13-\mathrm{C} 14$ & $117.97(14)$ \\
\hline $\mathrm{C} 3-\mathrm{C} 4-\mathrm{H} 4 \mathrm{~A}$ & 109.2 & $\mathrm{C} 18-\mathrm{C} 13-\mathrm{C} 12$ & $120.39(14)$ \\
\hline $\mathrm{C} 5-\mathrm{C} 4-\mathrm{H} 4 \mathrm{~B}$ & 109.2 & $\mathrm{C} 14-\mathrm{C} 13-\mathrm{C} 12$ & $121.63(14)$ \\
\hline $\mathrm{C} 3-\mathrm{C} 4-\mathrm{H} 4 \mathrm{~B}$ & 109.2 & $\mathrm{C} 15-\mathrm{C} 14-\mathrm{C} 13$ & $121.18(14)$ \\
\hline $\mathrm{H} 4 \mathrm{~A}-\mathrm{C} 4-\mathrm{H} 4 \mathrm{~B}$ & 107.9 & $\mathrm{C} 15-\mathrm{C} 14-\mathrm{H} 14$ & 119.4 \\
\hline $\mathrm{C} 6-\mathrm{C} 5-\mathrm{C} 4$ & $111.51(12)$ & $\mathrm{C} 13-\mathrm{C} 14-\mathrm{H} 14$ & 119.4 \\
\hline $\mathrm{C} 6-\mathrm{C} 5-\mathrm{H} 5 \mathrm{~A}$ & 109.3 & $\mathrm{C} 14-\mathrm{C} 15-\mathrm{C} 16$ & $119.93(14)$ \\
\hline $\mathrm{C} 4-\mathrm{C} 5-\mathrm{H} 5 \mathrm{~A}$ & 109.3 & $\mathrm{C} 14-\mathrm{C} 15-\mathrm{H} 15$ & 120.0 \\
\hline $\mathrm{C} 6-\mathrm{C} 5-\mathrm{H} 5 \mathrm{~B}$ & 109.3 & $\mathrm{C} 16-\mathrm{C} 15-\mathrm{H} 15$ & 120.0 \\
\hline $\mathrm{C} 4-\mathrm{C} 5-\mathrm{H} 5 \mathrm{~B}$ & 109.3 & $\mathrm{C} 15-\mathrm{C} 16-\mathrm{C} 17$ & $119.88(15)$ \\
\hline $\mathrm{H} 5 \mathrm{~A}-\mathrm{C} 5-\mathrm{H} 5 \mathrm{~B}$ & 108.0 & $\mathrm{C} 15-\mathrm{C} 16-\mathrm{C} 19$ & $119.24(14)$ \\
\hline $\mathrm{C} 7-\mathrm{C} 6-\mathrm{C} 11$ & $118.67(14)$ & $\mathrm{C} 17-\mathrm{C} 16-\mathrm{C} 19$ & $120.86(14)$ \\
\hline $\mathrm{C} 7-\mathrm{C} 6-\mathrm{C} 5$ & $119.49(13)$ & $\mathrm{C} 18-\mathrm{C} 17-\mathrm{C} 16$ & $119.40(15)$ \\
\hline $\mathrm{C} 11-\mathrm{C} 6-\mathrm{C} 5$ & $121.83(14)$ & $\mathrm{C} 18-\mathrm{C} 17-\mathrm{H} 17$ & 120.3 \\
\hline $\mathrm{C} 6-\mathrm{C} 7-\mathrm{C} 8$ & $121.55(15)$ & $\mathrm{C} 16-\mathrm{C} 17-\mathrm{H} 17$ & 120.3 \\
\hline $\mathrm{C} 6-\mathrm{C} 7-\mathrm{H} 7$ & 119.2 & $\mathrm{C} 17-\mathrm{C} 18-\mathrm{C} 13$ & $121.62(14)$ \\
\hline $\mathrm{C} 8-\mathrm{C} 7-\mathrm{H} 7$ & 119.2 & $\mathrm{C} 17-\mathrm{C} 18-\mathrm{H} 18$ & 119.2 \\
\hline $\mathrm{C} 7-\mathrm{C} 8-\mathrm{C} 9$ & $119.64(15)$ & $\mathrm{C} 13-\mathrm{C} 18-\mathrm{H} 18$ & 119.2 \\
\hline $\mathrm{C} 7-\mathrm{C} 8-\mathrm{H} 8$ & 120.2 & $\mathrm{~N} 1-\mathrm{C} 19-\mathrm{C} 16$ & $177.09(16)$ \\
\hline
\end{tabular}

Hydrogen-bond geometry $\left(\AA,{ }^{\circ}\right)$

\begin{tabular}{lllll}
\hline$D-\mathrm{H} \cdots A$ & $D-\mathrm{H}$ & $\mathrm{H} \cdots A$ & $D \cdots A$ & $D-\mathrm{H} \cdots A$ \\
\hline $\mathrm{C} 17-\mathrm{H} 17 \cdots \mathrm{N} 1^{\mathrm{i}}$ & 0.95 & 2.54 & $3.438(2)$ & 157 \\
$\mathrm{C} 3-\mathrm{H} 3 A \cdots C g 1^{\mathrm{ii}}$ & 0.99 & 2.84 & $3.6730(16)$ & 142 \\
$\mathrm{C} 8-\mathrm{H} 8 \cdots C g 1^{\mathrm{iii}}$ & 0.95 & 2.88 & $3.7868(17)$ & 161 \\
\hline
\end{tabular}

Symmetry codes: (i) $-x,-y-1,-z+1$; (ii) $x, y-1, z$; (iii) $-x+1, y+1 / 2,-z+1 / 2$. 